\title{
A Class of Nonzero-sum Investment and Reinsurance Games subject to Systematic Risks
}

\author{
Chi Chung Siu ${ }^{a}$, Sheung Chi Phillip Yam ${ }^{b}$, Hailiang Yang ${ }^{c}$, and Hui Zhao ${ }^{d}$ \\ ${ }^{a}$ Finance Discipline Group, Business School, University of Technology, Sydney, Sydney, \\ Australia.* \\ ${ }^{b}$ Department of Statistics, The Chinese University of Hong Kong Shatin, N.T., Hong Kong. ${ }^{\dagger}$ \\ ${ }^{c}$ Department of Statistics and Actuarial Science, The University of Hong Kong, Pokfulam Road, \\ Hong Kong. ${ }^{\ddagger}$ \\ ${ }^{d}$ School of Science, Tianjin University, Tianjin 300072, PR China ${ }^{\S}$
}

\begin{abstract}
Recently, there have been numerous insightful applications of zero-sum stochastic differential games in insurance, as discussed in Liu et al. (2014). While there could be some practical situations under which nonzero-sum game approach is more appropriate, the development of such approach within actuarial contexts remains rare in the existing literature. In this article, we study a class of nonzero-sum reinsurance-investment stochastic differential games between two competitive insurers subject to systematic risks described by a general compound Poisson risk model. Each insurer can purchase the excess-of-loss reinsurance to mitigate both systematic and idiosyncratic jump risks of the inter-arrival claims; and can invest in one risk-free asset and one risky asset whose price dynamics follows the famous Heston (1993) stochastic volatility model. The main objective of each insurer is to maximize the expected utility of his terminal surplus relative to that of his competitor. Dynamic programming principle for this class of nonzero-sum game problems leads to a non-canonical fixed-point problem of coupled non-linear integral-typed equations. Despite the complex structure, we establish the unique existence of the Nash equilibrium reinsurance-investment strategies and the corresponding value functions of the insurers in a representative example of the CARA insurers under a mild, time-independent condition. Furthermore, Nash equilibrium strategies and value functions admit closed forms. Numerical studies are also provided to illustrate the impact of the systematic risks on the Nash equilibrium strategies. Finally, we connect our results to that under the diffusion-approximated model by proving explicitly that the Nash equilibrium under the diffusion-approximated model is an $\epsilon$-Nash equilibrium under the general Poisson risk model, thereby establishing that the analogous Nash equilibrium in Bensoussan et al. (2014) serves as an interesting
\end{abstract}

*chichungsiu@uts.edu.au

†scpyam@sta.cuhk.edu.hk

†hlyang@hku.hk

§zhaohuimath@tju.edu.cn 
complementary case of the present framework.

Keywords: Nonzero-sum stochastic differential game; Systematic risks; Compound Poisson risk model; Excess-of-loss reinsurance; Heston stochastic volatility model; Nash equilibrium; Hamilton-Jacobi-Bellman (HJB) equation; Fixed-point problems; $\epsilon$-Nash equilibrium

\section{Introduction}

The seminal works of Gerber (1974) and Bühlmann (1974) have initiated the adoption of stochastic control techniques to study the optimal reinsurance, investment, and consumption problems of an insurer maximizing his absolute terminal wealth under different stochastic environments. Examples include Browne (1995) for establishing the equivalence between the maximization of the exponential terminal utility and the minimization of the probability of ruin in the absence of interest rate; Yang and Zhang (2005) for solving the explicit investment strategies for the case of the jump-diffusion process, and Liu and Ma (2009) for solving the optimal reinsurance-investment-consumption problem under a general semi-martingale setting; and Liu et al. (2013) for solving the optimal investment-reinsurance problem for an insurer subject to a dynamic risk constraint under a Markovian regime-switching environment.

Yet, insurers often make decisions to best assess his performance against a relative benchmark of his competitors, resulting in the study of the optimal strategy subject to the relative performance concerns. In this aspect, Elliott (1976) pioneered the study of the zero-sum stochastic differential game, in which one player selects a strategy to maximize the value function whereas the other player minimizes it, by examining the solvability of the zero-sum stochastic differential game with the establishment of the Isaac condition and its association to the existence of a saddle point for the game. For the applications of the zero-sum games in the contexts of the investment and reinsurance problems, see, for example, Browne (2000) on the stochastic differential investment game between two players; Zeng (2010) on the stochastic reinsurance game; Elliott and Siu (2011) on the zero-sum game formulation of a portfolio selection problem subject to the convex risk measure; Liu et al. (2014) on solving the expected exponential-utility maximization problem of an insurer subject to the worst-case probability risk by formulating the original maximization problem into a zero-sum stochastic differential game problem between an insurer and the market.

In reality, however, each insurer has different risk-tolerance, and the study of the relative performance concern becomes a nonzero-sum game problem. Unlike the case of the zerosum games, finding the Nash equilibria in a nonzero-sum game proves to be significantly challenging as it usually involves finding solutions to the coupled equations. Bensoussan and Friedman (1977) showed that the Nash equilibrium of a nonzero-sum stopping game between two players can be reduced to finding a solution of the corresponding quasi-variational inequality, which admits stationary solution. Bensoussan and Freshe (2000) employed the dynamic programming principle to show that the existence of the non-stationary Nash equilibria in a nonzero-sum stochastic differential game is equivalent to solving the strongly coupled system of partial differential equations (PDEs). For the applications of the nonzerosum games in economics, finance, and insurance, Hamadéne and Zhang (2009) established the Nash equilibrium of a nonzero-sum Dynkin game between two players in continuous time 
and adopted the approach to price American game contingent claims, and was later extended to the multi-player setting in Hamadéne and Hassani (2014); Bensoussan et al. (2014) for the establishment of the closed-form Nash equilibrium of the nonzero-sum reinsurance and investment game between two insurers under the mixed regime-switching framework by means of the dynamic programming principle; and Espinosa and Touzi (2015) for the adoption of the stochastic maximum principle to prove the existence of the non-stationary Nash equilibrium in a $N$-person game within the Brownian motion framework with deterministic coefficients.

In this paper, we provide the first study on the impact of the systematic risks on a class of nonzero-sum stochastic differential games subject to systematic risks under a general compound Poisson risk model. We model the surplus process of each of the two insurance companies as a jump-diffusion process, consisting of an excess-of-loss reinsurance protection and of an investment in risky and risk-free assets. Each insurance company chooses a reinsuranceinvestment strategy pair to maximize his utility of the difference between his terminal surplus and that of his competitor. Each insurer is exposed to two sources of systematic risks: 1) claim risks and 2) investment risk. More specifically, each insurer's surplus process is affected by the systematic as well as idiosyncratic jump risks of inter-arrival claims, whereas the systematic investment risk is derived from their investment on the same risky asset, whose dynamics follows the famous Heston (1993) stochastic volatility model. Although the most closely related work to ours is Bensoussan et al. (2014), the present paper substantially differs from the aforementioned work in four main aspects.

1. We illustrate the impact of volatility risk of the risky asset on the equilibrium investment strategies of both insurers. In Bensoussan et al. (2014), the regime-switching dynamics is independent of the dynamics of the risky asset. The direct consequence of their result is that the frequency of the regime-switch has no impact on the equilibrium strategies of both insurers, and hence the volatility (regime-dependent) process is always inversely proportional to the equilibrium investment strategy of each insurer. In contrast, we explicitly model the correlation between the risky asset and its volatility process by adopting the popular Heston (1993) stochastic volatility model, to quantify the volatility risk to the investment strategies of the insurers when they both invest in the same risky asset. We show that the sign of the correlation between the volatility and risky asset processes proves to be pivotal to the directional changes of the investment strategies of the insurers at equilibrium. In particular, when the correlation between the risky asset and its volatility process is negative, increasing investment on the risky asset can hedge against the surging volatility risk, and therefore both insurers would then choose to increase investment when the volatility risk is high. However, when the aforementioned correlation is positive, such hedging effect dissipates and therefore both insures would decrease their investment on the risky assets when exposing to rising volatility risk.

2. In addition to the consideration of the Cramer-Lundberg diffusion-approximated model, we model the surplus process of each insurance company based on the compound Poisson risk model, which is more realistic as the arrivals of claims are usually modelled as the Poisson processes. Moreover, we assume that the surplus process of each insurer has an idiosyncratic jump risk as well as the systematic jump risk. Instead of the purchasing the proportional reinsurance protection, as the case in Bensoussan et al. (2014), we consider the insurers choose to purchase the excess-of-loss reinsurance 
protection, in which they choose the retention level so that any loss in excess of that level would be covered by the reinsurance company. As discussed in Asmussen et al. (2000), the excess-of-loss reinsurance protection yields higher profit to the insurers than the proportional reinsurance protection, and thus are more popular to the insurance companies. In this respect, our nonzero-sum game model in this article quantifies the impact of competition to the optimal choice of the retention level of each insurer at equilibrium. More importantly, we highlight the importance of the systematic jump risk to the equilibrium reinsurance strategy by considering two extreme cases. In the case when there is no systematic jump risk, we show that the equilibrium reinsurance strategy of each insurer coincides with the classical optimal reinsurance strategy when there is no competition. In other words, the concept of nonzero-sum game is irrelevant to the insurers in the context of reinsurance when there is no systematic jump risk. On the other hand, when the intensity of systematic jump risk increases, the equilibrium reinsurance demand for each insurer decreases, i.e. their corresponding retention levels at equilibrium increase. As the systematic jump risk affects both insurers, increasing the purchase of the reinsurance protection would not improve the terminal relative surplus of each insurer but would rather deteriorate it, and therefore increasing the coverage of the reinsurance protection cannot be the best response to the strategy of his competitor. This result highlights the fundamental nature of systematic risk to the insurers subject to the relative performance concerns. Unlike the standard result from the optimal strategy subject to the absolute performance concern in which insurer would increase the risk-mitigating strategy in face of higher risk, insurers with the relative performance concern have different strategy for the idiosyncratic and the systematic risks. As idiosyncratic risk only affects his surplus and not the surplus of his competitor, an insurer would increase the risk-mitigation strategy to hedge against such risk. On the other hand, systematic risk affects the wealth of both insurers and hence each non-cooperative insurer would realize increasing the risk-mitigating measure on the systematic risk would reduce his relative terminal surplus. Therefore, the best response of each non-cooperative insurer would then reduce the coverage of the risktaking measure in face of surging systematic risk, and our result captures this important feature.

3. The realistic consideration of the excess-of-loss protection under the general compound Poisson risk model makes the methodology established in Bensoussan et al. (2014) to be non-implementable when establishing the Nash equilibrium reinsurance strategies in this article. Specifically, we use the representative example of two CARA insurers to show that the existence of the equilibrium excess-of-loss reinsurance strategies becomes the solution of the coupled non-linear integral-typed equations, as opposed to the coupled linear equations in Bensoussan et al. (2014). In other words, the fixed-point problem in this article proves to be non-trivial, and the solution of which has not been found in the existing literature. Nonetheless, we show that the equilibrium reinsurance strategies are unique under a mild, time-independent, condition. The condition is mild because it can be satisfied when the intensity of the systematic jump risk is small relative to the intensities of the idiosyncratic jump risks of the insurers. This is in agreement with reality as the insurers would choose reinsurance protection and investment strategies to diversify their risks so that the magnitude of the systematic risks would be small relative to the magnitude of the idiosyncratic risks under normal 
circumstances.

4. Finally, to best understand the connection between the compound Poisson risk model and the diffusion-approximated model under a stochastic differential game framework, which is the framework first adopted in Bensoussan et al. (2014) for the case of proportional reinsurance, we prove that the stochastic differential game under a compound Poisson risk process encompasses that under the diffusion-approximated process in the following sense. We first provide the explicit equilibrium excess-of-loss reinsurance/investment strategies under the diffusion-approximated model. Interestingly enough, the existence of the Nash equilibrium reinsurance-investment strategies under the diffusion model requires no additional condition, as opposed to the case of the compound Poisson model. This shows that the high-tractability of the diffusionapproximated model is maintained in the nonzero-sum game framework. In addition, we show that the Nash equilibrium under the compound Poisson risk process converges to that under its diffusion-approximated process; and the condition of existence of the Nash equilibrium under the compound Poisson model is satisfied asymptotically. More importantly, we show that the Nash equilibrium strategies, value functions, and the objective functions under the diffusion-approximated process can be arbitrary close to that under the compound Poisson under some appropriate technical condition. In other words, the Nash equilibrium under the diffusion-approximated model is the $\epsilon$ Nash equilibrium, of which the early notion can be found in the classical works such as Tijs (1981) and the references therein, for the general compound Poisson risk model. In other words, we provide the first theoretical justification for the adoption of the diffusion-approximated model in the nonzero-sum game framework.

The rest of the paper is organized as follows. Section 2 introduces the model dynamics of the two competing insurance companies. Section 3 presents the optimal reinsurance-investment problem subject to the relative performance concern. We show that this problem is equivalent to the nonzero-sum game between two insurers. Section 4 provides the characterization of the Nash equilibrium under the general utility function. Section 5 shows that in the case when both insurers have exponential utilities, the Nash equilibrium exists and admits closedform expression under a mild condition. Section 6 has two purposes: 1) we show that the Nash equilibrium under the CARA insurers exists unconditionally under the diffusionapproximated framework and that associated Nash equilibrium strategies and value functions also admit closed-form solutions; 2) we establish the Nash equilibrium under the diffusionapproximated model is an $\epsilon$-Nash equilibrium under the general compound Poisson model and that the corresponding mild condition for the existence of Nash equilibrium under the general Poisson model is satisfied asymptotically. Section 7 provides detailed numerical studies to discuss the impact of competition on the equilibrium strategies. Section 8 concludes the paper with some suggestions for future research. Appendix A contains the proof of the verification theorem for the case of two competing CARA insurers.

\section{Model formulation}

Let $(\Omega, \mathcal{F}, \mathbb{P})$ be a complete probability space. Here, we shall assume that $\mathcal{F}=\left(\mathcal{F}_{t}\right)_{t \geq 0}$, where $\mathcal{F}_{t} \triangleq \mathcal{F}_{t}^{X}$, where the processes $\left\{X(t) \triangleq\left(X_{1}^{\pi_{1}^{\mathfrak{n}}}(t), X_{2}^{\pi_{2}^{\mathfrak{n}}}(t)\right)\right\}_{t \geq 0}$ will be defined subsequently 
below. Denote by $\|f\|_{\infty}$ the uniform norm of the function $f$, i.e.

$$
\|f\|_{\infty} \triangleq \sup \{|f(x)|: x \in \mathcal{D}\}, \quad \text { for } \quad \mathcal{D} \subset \mathbb{R} .
$$

\subsection{Surplus process}

We begin with the specifications of the surplus processes of two competing insurance companies, having dependent lines of the insurance business. The surplus process of insurer $k$, denoted by $\left\{R_{k}^{\mathfrak{n}}(t)\right\}_{t \geq 0}$, for $k=1,2$, is represented by the classical risk model, i.e.,

$$
\mathrm{d} R_{k}^{\mathfrak{n}}(t)=c_{k} \mathrm{~d} t-\mathrm{d}\left(C(\mathfrak{n}) \sum_{i=1}^{N_{k}\left(\mathfrak{n}^{\mathfrak{a}} t\right)+N\left(\mathfrak{n}^{\mathfrak{a}} t\right)} Z_{i}^{k}\right), \quad k=1,2
$$

where $c_{k}$ is the premium rate for $k=1,2 ; \mathfrak{a} \geq 1$ and $C$ is a positive scaling function of $\mathfrak{n} \in \mathbb{N}$ such that $C(1)=1$ and $\lim _{\mathfrak{n} \rightarrow \infty}(C(\mathfrak{n}))^{2} \mathfrak{n}^{\mathfrak{a}}$ exists; and $\left\{N_{1}(t)\right\}_{t \geq 0},\left\{N_{2}(t)\right\}_{t \geq 0}$ and $\{N(t)\}_{t \geq 0}$ are three mutually independent Poisson processes with intensities $\lambda_{1}>0, \lambda_{2}>0$, and $\lambda>0$, respectively. ${ }^{1}$ The surplus process $\left\{R_{k}^{\mathfrak{n}}(t)\right\}_{t \geq 0}$ in (1) indicates that each insurer is subject to a common systematic insurance risk, represented as $N(t)$, in addition to his idiosyncratic insurance risk, represented as $N_{k}(t)$, for $k=1,2$, at any time $t>0$. The claim sizes $\left\{Z_{i}^{k}, i=1,2, \ldots\right\}, k=1,2$ are independent and identically distributed positive random variables with common distribution $F_{k}(z)$, and we shall hereafter use $Z^{k}$ as the claim size facec by insurer $k$, for $k=1,2$, in place of $Z_{i}^{k}$ for more transparent presentation. Let $D_{k} \triangleq \sup \left\{z: F_{k}(z) \leq 1\right\}$ and suppose that $F_{k}(0)=0,0<F_{k}(z)<1$, for $0<z<D_{k}$, and $F_{k}(z)=1$ for $z \geq D_{k}$. Denote by $\bar{\mu}_{k} \triangleq \mathbb{E}\left[Z_{i}^{k}\right]$. In addition, we assume that $\left\{Z_{i}^{1}, i=1,2, \ldots\right\}$ and $\left\{Z_{i}^{2}, i=1,2, \ldots\right\}$ are mutually independent and that both of them are independent of $\left\{N_{k}(t)\right\}_{t \geq 0}$, for $k=1,2$ and $\{N(t)\}_{t \geq 0}$. Suppose also that the premium is calculated according to the expected value principle, i.e., $c_{k}=\left(C(\mathfrak{n}) \mathfrak{n}^{\mathfrak{a}}+\eta_{k}\right)\left(\lambda_{k}+\lambda\right) \bar{\mu}_{k}$, where $\eta_{k}>0$ is the safety loading of insurer $k$, for $k=1,2$, used to cover his own costs of operations. With these notations, the surplus process $\left\{R_{k}^{\mathfrak{n}}(t)\right\}_{t \geq 0}$, for $k=1,2$, can be rewritten as

$$
\mathrm{d} R_{k}^{\mathfrak{n}}(t)=\eta_{k}\left(\lambda_{k}+\lambda\right) \bar{\mu}_{k} \mathrm{~d} t-\mathrm{d}\left(C(\mathfrak{n})\left[\left(N_{k}\left(\mathfrak{n}^{\mathfrak{a}} t\right)+N\left(\mathfrak{n}^{\mathfrak{a}} t\right)\right) Z^{k}-\mathfrak{n}^{\mathfrak{a}}\left(\lambda_{k}+\lambda\right) \bar{\mu}_{k} t\right]\right) .
$$

Remark 2.1. Note that the limit process of $\left\{R_{k}^{\mathfrak{n}}(t)\right\}_{t \geq 0}$, i.e. $\lim _{\mathfrak{n} \rightarrow \infty}\left\{R_{k}^{\mathfrak{n}}(t)\right\}_{t \geq 0}$, is welldefined by the virtue of the martingale central limit theorem (see, for example, Theorem 1.4 of Chapter 7 in Ethier and Kurtz (1986)). Same remarks apply to the processes $\left\{R_{k}^{a_{k}^{\mathrm{n}}}(t)\right\}_{t \geq 0}$ in (2) and $\left\{X_{k}^{\pi_{k}^{\mathfrak{n}}}(t)\right\}_{t \geq 0}$ in (4).

\subsection{Reinsurance and investment opportunities}

Suppose that each insurer can mitigate his insurance risks with a purchase of an excess-ofloss reinsurance protection. More precisely, let $\left\{a_{k}^{\mathfrak{n}}(t)\right\}_{t \geq 0}$ be a $\mathcal{F}$-progressively measurable process representing a (fixed) excess-of-loss retention level for insurer $k$, for $k=1,2$. Note that under the assumption on $F_{k}, \mathbb{E}\left[\left(Z_{i}^{k}\right)^{2}\right]<\infty$ and hence we have $a_{k}^{\mathfrak{n}} \in \mathcal{L}^{2}(\mathbb{R})$, for $k=1,2$.

\footnotetext{
${ }^{1}$ The same result applies if the claim size distributions are different for the idiosyncratic $\left\{N_{k}(t)\right\}_{t \geq 0}$, for $k=1,2$, and systematic $\{N(t)\}_{t \geq 0}$ shocks.
} 
Let $\mathbb{A}_{k} \triangleq\left\{a_{k}^{\mathfrak{n}}(t) \in \mathcal{L}^{2}(\mathbb{R}), t \geq 0\right\}$ be the set of excess-of-loss retention levels of insurer $k$. The corresponding surplus process with the reinsurance protection, denoted by $\left\{R_{k}^{a_{k}^{\mathrm{n}}}(t)\right\}_{t \geq 0}$, for $k=1,2$, then becomes

$$
\begin{aligned}
\mathrm{d} R_{k}^{a_{k}^{\mathfrak{n}}}(t)= & \left(\lambda_{k}+\lambda\right)\left[\theta_{k} \mu_{k}\left(a_{k}^{\mathfrak{n}}\right)+\left(\eta_{k}-\theta_{k}\right) \bar{\mu}_{k}\right] \mathrm{d} t \\
& -\mathrm{d}\left(C(\mathfrak{n})\left[\left(N_{k}\left(\mathfrak{n}^{\mathfrak{a}} t\right)+N\left(\mathfrak{n}^{\mathfrak{a}} t\right)\right)\left(Z^{k} \wedge a_{k}^{\mathfrak{n}}\right)-\left(\lambda_{k}+\lambda\right) \mathfrak{n}^{\mathfrak{a}} \mu_{k}\left(a_{k}^{\mathfrak{n}}\right) t\right]\right),
\end{aligned}
$$

where $x \wedge y \triangleq \min (x, y), \mu_{k}\left(a_{k}^{\mathfrak{n}}\right)=\mathbb{E}\left[\left(Z^{k} \wedge a_{k}^{\mathfrak{n}}\right)\right]=\int_{0}^{a_{k}^{\mathfrak{n}}} \bar{F}_{k}(x) \mathrm{d} x$, for $\bar{F}_{k}(x) \triangleq 1-F_{k}(x)$, and $\theta_{k}$ denotes the safety loading of the reinsurer for insurer $k$. As it is more common in practice that the reinsurer requests higher safety loading from the insurers, we shall hereafter assume that $\theta_{k}>\eta_{k}$, for $k=1,2$, namely non-cheap reinsurance protection. From the reinsuranceprotected surplus process of insurer $k,\left\{R_{k}^{a_{k}^{\mathfrak{n}}}(t)\right\}_{t \geq 0}$, in (2), insurer $k$ selects the retention level $a_{k}$ such that he only needs to pay all claims up to the fixed amount $a_{k}^{\mathfrak{n}}$, while all in excess of $a_{k}$ will be covered by the reinsurance company.

In addition to purchasing the reinsurance protection, the insurer can invest in a stock market. More specifically, assume that each insurer can invest in a risk-free asset,and a risky asset. The price process of the risk-free asset is given by

$$
\mathrm{d} S_{0}(t)=r S_{0}(t) \mathrm{d} t
$$

where $r>0$ is the risk-free interest rate. To capture the impact of the volatility risk to the insurers' strategies, we model the price process of the risky asset by the Heston (1993) stochastic volatility model, i.e.

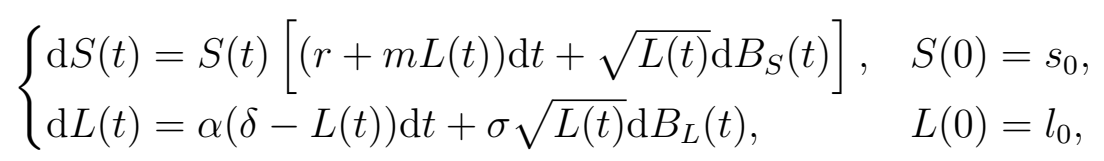

where $m, \alpha, \delta$ and $\sigma$ are all positive constants, $\left\{B_{S}(t)\right\}_{t \geq 0}$ and $\left\{B_{L}(t)\right\}_{t \geq 0}$ are two standard Brownian motions with $\mathrm{E}\left[B_{S}(t) B_{L}(t)\right]=\rho_{S} t$. Moreover, we require that $2 \alpha \delta \geq \sigma^{2}$ (see Cox et al. (1985)).

Let $\left\{b_{k}^{\mathfrak{n}}(t)\right\}_{t \geq 0}$ be the $\mathcal{F}$-progressively measurable process representing the money amount that insurer $k$ invests in the risky asset $S$, for $k=1,2$. Define $\mathbb{B}_{k} \triangleq\left\{b_{k}^{\mathfrak{n}}(t), t \geq 0\right\}$ as the set of investment amounts of insurer $k$. A reinsurance-investment strategy of insurer $k$ is then denoted as $\left\{\pi_{k}^{\mathfrak{n}}(t)\right\}_{t \geq 0} \triangleq\left\{\left(a_{k}^{\mathfrak{n}}(t), b_{k}^{\mathfrak{n}}(t)\right)\right\}_{t \geq 0} \in \mathbb{A}_{k} \times \mathbb{B}_{k}$. Corresponding to a reinsuranceinvestment strategy $\pi_{k}^{\mathfrak{n}}(t)$, the surplus process of insurer $k$, denoted by $\left\{X_{k}^{\pi_{k}^{\mathfrak{n}}}(t)\right\}_{t \geq 0}$, for $k=1,2$, takes the form

$$
\left\{\begin{aligned}
\mathrm{d} X_{k}^{\pi_{k}^{\mathfrak{n}}}(t)= & \left\{\left(\lambda_{k}+\lambda\right)\left[\theta_{k} \mu_{k}\left(a_{k}^{\mathfrak{n}}\right)+\left(\eta_{k}-\theta_{k}\right) \bar{\mu}_{k}\right]+r X_{k}^{\pi_{k}^{\mathfrak{n}}}(t)+m b_{k}^{\mathfrak{n}}(t) L(t)\right\} \mathrm{d} t \\
& +b_{k}^{\mathfrak{n}}(t) \sqrt{L(t)} \mathrm{d} B_{S}(t)-C(\mathfrak{n}) \mathrm{d}\left[\left(N_{k}\left(\mathfrak{n}^{\mathfrak{a}} t\right)+N\left(\mathfrak{n}^{\mathfrak{a}} t\right)\right)\left(Z^{k} \wedge a_{k}^{\mathfrak{n}}\right)\right. \\
& \left.-\left(\lambda_{k}+\lambda\right) \mathfrak{n}^{\mathfrak{a}} \mu_{k}\left(a_{k}^{\mathfrak{n}}\right) t\right] \\
X_{k}^{\pi_{k}^{\mathfrak{n}}}(0)= & x_{k} .
\end{aligned}\right.
$$

We denote by $\Pi_{k} \triangleq \mathcal{A}_{k} \times \mathcal{B}_{k} \subset \mathbb{A}_{k} \times \mathbb{B}_{k}$ the set of convex strategies $\pi_{k}^{\mathfrak{n}}=\left(a_{k}^{\mathfrak{n}}, b_{k}^{\mathfrak{n}}\right) \in \Pi_{k}$ of insurer $k \in\{1,2\}$ satisfying condition that $a_{k}^{\mathfrak{n}} \in \mathcal{L}^{2}(\mathbb{R})$ and $\mathbb{E}\left[\int_{0}^{T}\left(b_{k}^{\mathfrak{n}}(t)\right)^{4} L^{2}(t) \mathrm{d} t\right]<\infty$, for all $T<\infty$. We shall refer a strategy $\pi_{k}^{\mathfrak{n}} \in \Pi_{k}$ to be an admissible strategy. From the 
standard theory of Lévy processes, (see, for example, Sato (1999)), it is clear that for all $\pi_{k}^{\mathfrak{n}}=\left(a_{k}^{\mathfrak{n}}, b_{k}^{\mathfrak{n}}\right) \in \Pi_{k}$, and for any initial condition $(t, x) \in[0, T] \times \mathbb{R}$, the stochastic differential equation $\left\{X_{k}^{\pi_{k}^{\mathfrak{n}}}(t)\right\}_{t \geq 0}$ in (4) admits a unique strong solution. In this case, for each $\mathfrak{n} \in \mathbb{N}$, Doob's $L^{p}$-inequality also gives

$$
\mathbb{E}\left[\sup _{0 \leq t \leq T}\left|X_{k}^{\pi_{k}^{\mathfrak{n}}}(t)\right|^{2}\right]<\infty, \quad \text { for } \quad k=1,2 .
$$

\section{Optimal strategy subject to the relative performance concerns}

Suppose that insurer $k$, for $k=1,2$ has a utility function $U^{k}$ which is strictly concave and continuously differentiable on $(-\infty, \infty)$. The optimization problem of each insurer is to optimally choose a reinsurance-investment strategy, $\pi_{k}^{\mathfrak{n}} \in \Pi_{k}$, for $k=1$, 2, that maximizes the expected utility of his relative performance against that of his competitor at the terminal time T. More precisely, we have

Problem 3.1. The optimal reinsurance and investment problem subject to relative performance concern between two insurance companies under the expected utility framework is a coupled stochastic optimization problem such that:

Given the strategy of his competitor $j \in\{1,2\}$, i.e. $\pi_{j}^{\mathfrak{n}}=\left(a_{j}^{\mathfrak{n}}, b_{j}^{\mathfrak{n}}\right)$, the objective function, denote as $J_{k}^{\mathfrak{n}}\left(t, x_{k}, x_{j}, \pi_{k}^{\mathfrak{n}}(t), \pi_{j}^{\mathfrak{n}}(t)\right)$, of insurer $k \neq j$ is to find an optimal strategy $\hat{\pi}_{k}^{\mathfrak{n}} \triangleq\left(\hat{a}_{k}^{\mathfrak{n}}, \hat{b}_{k}^{\mathfrak{n}}\right)$ such that

$$
\max _{\pi_{k}^{\mathfrak{n}}=\left(a_{k}^{\mathfrak{n}}, b_{k}^{\mathfrak{n}}\right) \in \Pi_{k}} J_{k}^{\mathfrak{n}}\left(t, x_{k}, x_{j}, \pi_{k}^{\mathfrak{n}}(t), \pi_{j}^{\mathfrak{n}}(t)\right)=J_{k}^{\mathfrak{n}}\left(t, x_{k}, x_{j}, \hat{\pi}_{k}^{\mathfrak{n}}(t), \pi_{j}^{\mathfrak{n}}(t)\right)
$$

where

$$
\begin{aligned}
& J_{k}^{\mathfrak{n}}\left(t, x_{k}, x_{j}, \pi_{k}^{\mathfrak{n}}(t), \pi_{j}^{\mathfrak{n}}(t)\right) \\
\triangleq & \mathbb{E}\left[U^{k}\left(\left(1-\kappa_{k}\right) X_{k}^{\pi_{k}^{\mathfrak{n}}}(T)+\kappa_{k}\left(X_{k}^{\pi_{k}^{\mathfrak{n}}}(T)-X_{j}^{\pi_{j}^{\mathfrak{n}}}(T)\right)\right) \mid X_{k}^{\pi_{k}^{\mathfrak{n}}}(t)-\kappa_{k} X_{j}^{\pi_{j}^{\mathfrak{n}}}(t)=x, L(t)=l\right] \\
= & \mathbb{E}\left[U^{k}\left(X_{k}^{\pi_{k}^{\mathfrak{n}}}(T)-\kappa_{k} X_{j}^{\pi_{j}^{\mathfrak{n}}}(T)\right) \mid X_{k}^{\pi_{k}^{\mathfrak{n}}}(t)-\kappa_{k} X_{j}^{\pi_{j}^{\mathfrak{n}}}(t)=x, L(t)=l\right] .
\end{aligned}
$$

The parameter $\kappa_{k} \in[0,1]$ in (6), for $k=1,2$, can be interpreted as the sensitivity parameter of insurer $k$ to his/her competitor's performance. As different insurer generally has different perception on the degree of influence of their competitor's surplus on his strategy, i.e. $\kappa_{1} \neq \kappa_{2}$, Problem 3.1 is a typical example of the nonzero-sum games between two insurers. Consequently, the solution to Problem 3.1 is the Nash equilibrium of the nonzero-sum game between two insurers, where the Nash equilibrium is the strategy $\pi_{k}^{*, \mathfrak{n}} \triangleq\left(a_{k}^{*, \mathfrak{n}}, b_{k}^{*, \mathfrak{n}}\right) \in \Pi_{k}$ such that, for all $\hat{\pi}_{1}^{\mathfrak{n}} \triangleq\left(\hat{a}_{1}^{\mathfrak{n}}, \hat{b}_{1}^{\mathfrak{n}}\right)$ and $\hat{\pi}_{2}^{\mathfrak{n}} \triangleq\left(\hat{a}_{2}^{\mathfrak{n}}, \hat{b}_{2}^{\mathfrak{n}}\right)$,

$$
\left\{\begin{array}{l}
J_{1}^{\mathfrak{n}}\left(t, x_{1}, x_{2}, \hat{\pi}_{1}^{\mathfrak{n}}(t), \pi_{2}^{*, \mathfrak{n}}(t)\right) \leq J_{1}^{\mathfrak{n}}\left(t, x_{1}, x_{2}, \pi_{1}^{*, \mathfrak{n}}(t), \pi_{2}^{*, \mathfrak{n}}(t)\right), \\
J_{2}^{\mathfrak{n}}\left(t, x_{2}, x_{1}, \hat{\pi}_{2}^{\mathfrak{n}}(t), \pi_{1}^{*, \mathfrak{n}}(t)\right) \leq J_{2}^{\mathfrak{n}}\left(t, x_{2}, x_{1}, \pi_{2}^{*, \mathfrak{n}}(t), \pi_{1}^{*, \mathfrak{n}}(t)\right) .
\end{array}\right.
$$




\section{Nash equilibrium for insurers in compound Poisson risk model: General case}

In this section, we solve for the Nash equilibrium of Problem 3.1 via the dynamic programming principle. To this end, denote by $\hat{X}_{k}^{\pi_{k}^{\mathfrak{n}}}(t) \triangleq X_{k}^{\pi_{k}^{\mathfrak{n}}}(t)-\kappa_{k} X_{j}^{\pi_{j}^{\mathfrak{n}}}(t)$ the relative surplus process of insurer $k$, for $k=1,2$, hence it follows that

$$
\left\{\begin{aligned}
\mathrm{d} \hat{X}_{k}^{\pi_{k}^{\mathfrak{n}}}(t)= & \left\{r \hat{X}_{k}^{\pi_{k}^{\mathfrak{n}}}(t)+\left(\lambda_{k}+\lambda\right)\left[\theta_{k} \mu_{k}\left(a_{k}^{\mathfrak{n}}(t)\right)\right.\right. \\
& \left.+\left(\eta_{k}-\theta_{k}\right) \bar{\mu}_{k}\right]-\kappa_{k}\left(\lambda_{j}+\lambda\right)\left[\theta_{j} \mu\left(a_{j}^{\mathfrak{n}}(t)\right)+\left(\eta_{j}-\theta_{j}\right) \bar{\mu}_{j}\right] \\
& \left.+m\left[b_{k}^{\mathfrak{n}}(t)-\kappa_{k} b_{j}^{\mathfrak{n}}(t)\right] L(t)\right\} \mathrm{d} t+\left[b_{k}^{\mathfrak{n}}(t)-\kappa_{k} b_{j}^{\mathfrak{n}}(t)\right] \sqrt{L(t)} \mathrm{d} B_{S}(t) \\
& -C(\mathfrak{n}) \mathrm{d}\left[\left(N_{k}\left(\mathfrak{n}^{\mathfrak{a}} t\right)+N\left(\mathfrak{n}^{\mathfrak{a}} t\right)\right)\left(Z^{k} \wedge a_{k}^{\mathfrak{n}}\right)-\left(\lambda_{k}+\lambda\right) \mathfrak{n}^{\mathfrak{a}} \mu_{k}\left(a_{k}^{\mathfrak{n}}(t)\right) t\right] \\
& +\kappa_{k} C(\mathfrak{n}) \mathrm{d}\left[\left(N_{j}\left(\mathfrak{n}^{\mathfrak{a}} t\right)+N\left(\mathfrak{n}^{\mathfrak{a}} t\right)\right)\left(Z^{j} \wedge a_{j}^{\mathfrak{n}}\right)-\left(\lambda_{j}+\lambda\right) \mathfrak{n}^{\mathfrak{a}} \mu_{j}\left(a_{j}^{\mathfrak{n}}(t)\right) t\right], \\
\hat{X}_{k}^{\pi_{k}^{\mathfrak{n}}}(0)= & \hat{x}_{k},
\end{aligned}\right.
$$

where $k \neq j \in\{1,2\}$. Define $V^{k, \mathfrak{n}}(t, x, l)$, for $k=1,2$, to be the value function as follows,

$$
V^{k, \mathfrak{n}}(t, x, l) \triangleq \sup _{\pi_{k}^{\mathfrak{n}} \in \Pi_{k}} \mathbb{E}\left[U^{k}\left(\hat{X}_{k}^{\pi_{k}^{\mathfrak{n}}}(T) \mid \hat{X}_{k}^{\pi_{k}^{\mathfrak{n}}}(t)=x, L(t)=l\right]\right.
$$

for $k, j=1,2$ with $k \neq j$. In addition, let $\Gamma=[0, T) \times \mathbb{R} \times \mathbb{R}^{+}$and $\bar{\Gamma}=[0, T] \times \mathbb{R} \times \mathbb{R}^{+}$; for $W^{k, \mathfrak{n}} \in \mathscr{C}^{1,2}(\Gamma) \cap \mathscr{C}^{0}(\bar{\Gamma})$, where $k \neq j \in\{1,2\}$, denote $\mathcal{L}^{\pi_{k}^{\mathfrak{n}}}$ to be the infinitesimal generator of $\hat{X}_{k}^{\pi_{k}^{\mathfrak{n}}}$ with an admissible strategy of insurer $k, \pi_{k}^{\mathfrak{n}} \in \Pi_{k}$.

$$
\begin{aligned}
\mathcal{L}^{\pi_{k}^{\mathfrak{n}}} W^{k, \mathfrak{n}}(t, x, l) \triangleq & \left\{r x+\left(\lambda_{k}+\lambda\right)\left[\left(\mathfrak{n}^{\mathfrak{a}} C(\mathfrak{n})+\theta_{k}\right) \mu_{k}\left(a_{k}^{\mathfrak{n}}\right)+\bar{\mu}^{k}\left(\eta_{k}-\theta_{k}\right)\right]\right. \\
& -\kappa_{k}\left(\lambda_{j}+\lambda\right)\left[\left(\mathfrak{n}^{\mathfrak{a}} C(\mathfrak{n})+\theta_{j}\right) \mu_{j}\left(a_{j}^{*, \mathfrak{n}}\right)+\bar{\mu}^{j}\left(\eta_{j}-\theta_{j}\right)\right] \\
& \left.+m l\left[b_{k}^{\mathfrak{n}}(t)-\kappa_{k} b_{j}^{*, \mathfrak{n}}(t)\right]\right\} W_{x}^{k, \mathfrak{n}}(t, x, l) \\
& +\frac{l}{2}\left[\left(b_{k}^{\mathfrak{n}}(t)\right)^{2}+\kappa_{k}^{2}\left(b_{j}^{*, \mathfrak{n}}(t)\right)^{2}-2 \kappa_{k} b_{k}^{\mathfrak{n}}(t) b_{j}^{*, \mathfrak{n}}(t)\right] W_{x x}^{k, \mathfrak{n}}(t, x, l) \\
& +\alpha(\delta-l) W_{l}^{k, \mathfrak{n}}(t, x, l)+\frac{1}{2} \sigma^{2} l W_{l l}^{k, \mathfrak{n}}(t, x, l) \\
& +\rho_{S} \sigma l\left[b_{k}^{\mathfrak{n}}(t)-\kappa_{k} b_{j}^{*, \mathfrak{n}}(t)\right] W_{x l}^{k, \mathfrak{n}}(t, x, l) \\
& +\mathfrak{n}^{\mathfrak{a}} \lambda_{k} \mathbb{E}\left[W^{k, \mathfrak{n}}\left(t, x-C(\mathfrak{n})\left(Z^{k} \wedge a_{k}^{\mathfrak{n}}(t)\right)\right)-W^{k, \mathfrak{n}}(t, x, l)\right] \\
& +\mathfrak{n}^{\mathfrak{a}} \lambda_{j} \mathbb{E}\left[W^{k, \mathfrak{n}}\left(t, x+\kappa_{k} C(\mathfrak{n})\left(Z^{j} \wedge a_{j}^{*, \mathfrak{n}}(t)\right)\right)-W^{k, \mathfrak{n}}(t, x, l)\right] \\
& +\mathfrak{n}^{\mathfrak{a}} \lambda \mathbb{E}\left[W^{k, \mathfrak{n}}\left(t, x-C(\mathfrak{n})\left(Z^{k} \wedge a_{k}^{\mathfrak{n}}(t)\right)+\kappa_{k} C(\mathfrak{n})\left(Z^{j} \wedge a_{j}^{*, \mathfrak{n}}(t)\right)\right)\right. \\
& \left.-W^{k, \mathfrak{n}}(t, x, l)\right] .
\end{aligned}
$$

Analogous to Kraft (2005), Taskar and Zeng (2013), and Zhao et al. (2013), we begin with the following verification theorem. 
Theorem 4.1. Let $W^{k, \mathfrak{n}} \in \mathscr{C}^{1,2}(\Gamma) \cap \mathscr{C}^{0}(\bar{\Gamma})$, for $k=1,2$, be the solution of the following Hamilton-Jacobi-Bellman (HJB) equation

$$
\left\{\begin{array}{l}
0=W_{t}^{k, \mathfrak{n}}(t, x, l)+\sup _{\pi_{k}^{\mathfrak{n}} \in \Pi_{k}} \mathcal{L}^{\pi_{k}^{\mathfrak{n}}} W^{k, \mathfrak{n}}(t, x, l), \quad \text { for } \quad 0 \leq t<T, \\
W^{k, \mathfrak{n}}(T, x, l)=U^{k}(x) .
\end{array}\right.
$$

For the relative surplus process $\hat{X}_{k}^{\pi_{k}^{\mathfrak{n}}}$ associated with an admissible strategy $\pi_{k}^{\mathfrak{n}} \in \Pi_{k}=\mathcal{A}_{k} \times \mathcal{B}_{k}$, we have

$$
\mathbb{E}\left[U^{k}\left(\hat{X}_{k}^{\pi_{k}^{\mathfrak{n}}}(T)\right) \mid \hat{X}_{k}^{\pi_{k}^{\mathfrak{n}}}(t)=x, L(t)=l\right] \leq W^{k, \mathfrak{n}}(t, x, l)
$$

Denote $\pi_{k}^{*}=\left(a_{k}^{*, \mathfrak{n}}, b_{k}^{*, \mathfrak{n}}\right)$, where $a_{k}^{*, \mathfrak{n}} \in \mathcal{A}_{k}, b_{k}^{*, \mathfrak{n}} \in \mathcal{B}_{k}$ are given as follows

$$
\begin{aligned}
a_{k}^{*, \mathfrak{n}}(t) \triangleq \underset{a_{k}^{\mathfrak{n}} \in \mathcal{A}_{k}}{\arg \max }\{ & \left(\lambda_{k}+\lambda\right)\left(\mathfrak{n}^{\mathfrak{a}} C(\mathfrak{n})+\theta_{k}\right) \mu_{k}\left(a_{k}^{\mathfrak{n}}(t)\right) W_{x}^{k, \mathfrak{n}}(t, x, l)+\lambda_{k} \mathfrak{n}^{\mathfrak{a}} \mathbb{E}\left[W^{k, \mathfrak{n}}(t, x\right. \\
& \left.\left.-\left(Z^{k} \wedge a_{k}^{\mathfrak{n}}(t)\right), l\right)-W^{k, \mathfrak{n}}(t, x, l)\right] \\
+\lambda \mathfrak{n}^{\mathfrak{a}} \mathbb{E}[ & W^{k, \mathfrak{n}}\left(t, x-C(\mathfrak{n})\left(Z^{k} \wedge a_{k}^{\mathfrak{n}}(t)\right)\right. \\
& \left.\left.\left.+\kappa_{k} C(\mathfrak{n})\left(Z^{j} \wedge a_{j}^{*, \mathfrak{n}}(t)\right), l\right)-W^{k, \mathfrak{n}}(t, x, l)\right]\right\}, \\
b_{k}^{*, \mathfrak{n}}(t) \triangleq \underset{b_{k}^{\mathfrak{n}} \in \mathcal{B}_{k}}{\arg \max }\{ & m l b_{k}^{\mathfrak{n}}(t) W_{x}^{k, \mathfrak{n}}(t, x, l)+\frac{l}{2}\left[\left(b_{k}^{\mathfrak{n}}(t)\right)^{2}-2 \kappa_{k} b_{k}^{\mathfrak{n}}(t) b_{j}^{*, \mathfrak{n}}(t)\right] W_{x x}^{k, \mathfrak{n}}(t, x, l) \\
& \left.+\rho_{S} \sigma l b_{k}^{\mathfrak{n}}(t) W_{x l}^{k, \mathfrak{n}}(t, x, l)\right\},
\end{aligned}
$$

and let $\hat{X}_{k}^{\pi_{k}^{*, n}}$ be the corresponding relative surplus process of insurer $k$. If for all sequences of stopping times $\left\{\tau_{i}\right\}_{i \in \mathbb{N}}$, the sequence $\left\{W^{k, \mathfrak{n}}\left(\tau_{i} \wedge T, \hat{X}_{k}^{\pi^{*, \mathfrak{n}}}\left(\tau_{i} \wedge T\right), L\left(\tau_{i} \wedge T\right)\right)\right\}_{i \in \mathbb{N}}$ is uniformly integrable, then we have

$$
V^{k, \mathfrak{n}}(t, x, l)=\mathbb{E}\left[U^{k}\left(\hat{X}_{k}^{\pi_{k}^{*, \mathfrak{n}}}(T)\right) \mid \hat{X}_{k}^{\pi_{k}^{*, \mathfrak{n}}}(t)=x, L(t)=l\right]=W^{k, \mathfrak{n}}(t, x, l) .
$$

In view of Theorem 4.1, the Nash equilibrium in (40) can now be formulated in terms the partial differential equations (PDEs). More specifically, we have

Theorem 4.2. Let $\hat{x}_{k}=x_{k}-\kappa_{k} x_{j}$, for $k, j \in\{1,2\}$ with $k \neq j$. The Nash equilibrium strategy for insurer $k$, for $k=1,2$, of Problem 3.1 is $\pi_{k}^{*, \mathfrak{n}}=\left(a_{k}^{*, \mathfrak{n}}, b_{k}^{*, \mathfrak{n}}\right)$, where $a_{k}^{*, \mathfrak{n}}, b_{k}^{*, \mathfrak{n}}$ admit the forms in (13) and (14), respectively; and the corresponding equilibrium value function is the solution to the following system of coupled PDEs:

$$
\left\{\begin{array}{l}
0=W_{t}^{1, \mathfrak{n}}\left(t, \hat{x}_{1}, l\right)+\mathcal{L}^{\pi_{1}^{*, \mathfrak{n}}} W^{1, \mathfrak{n}}\left(t, \hat{x}_{1}, l\right) \\
0=W_{t}^{2, \mathfrak{n}}\left(t, \hat{x}_{2}, l\right)+\mathcal{L}^{\pi_{2}^{*, \mathfrak{n}}} W^{2, \mathfrak{n}}\left(t, \hat{x}_{2}, l\right)
\end{array}\right.
$$

with the terminal conditions

$$
\left\{\begin{array}{l}
W^{1, \mathfrak{n}}\left(T, \hat{x}_{1}, l\right)=U^{1}\left(\hat{x}_{1}\right) \\
W^{2, \mathfrak{n}}\left(T, \hat{x}_{2}, l\right)=U^{2}\left(\hat{x}_{2}\right)
\end{array}\right.
$$


Due to the strongly coupling feature, the existence of solution to the PDE system in (16) is difficult to establish in general, as it is closely related to the regularity conditions of the system, see Bensoussan and Freshe (2000) for details. Since the compound Poisson risk process in (4) possesses finite number of jumps with all finite moments on any compact time interval $[0, T]$, together with some implied scaling structures, we can follow similar ideas as in the proof of the famous Cauchy-Kowalevski Theorem via the use of formal power series expansion and analytic majorization, to especially deal with the expectation terms in (16), and to establish the existence of its solution for very small $T$. Nonetheless, we show that for the representative case of the exponential utility function, we can establish uniquely existence of the solution to the system (16).

\section{Nash equilibrium for insurers in compound Poisson risk model: Case of CARA insurers}

For the rest of the paper, we shall focus on a representative case in which both insurers have constant-absolute-risk-aversion (CARA) preferences, i.e.

$$
U^{k}(x)=-\frac{1}{q_{k}} \mathrm{e}^{-q_{k} x},
$$

with $q_{k}>0, k=1,2$. Denote

$$
h_{k}^{\mathfrak{n}}(a) \triangleq-\int_{0}^{a} \kappa_{k} q_{k} \mathrm{e}^{r(T-t)} C(\mathfrak{n}) \mathrm{e}^{-\kappa_{k} q_{k} \mathrm{e}^{r(T-t)} C(\mathfrak{n}) y} \bar{F}_{j}(y) \mathrm{d} y,
$$

for $k \neq j \in\{1,2\}$. The following theorem provides the closed-form expressions for the Nash equilibrium between two CARA insurers.

Theorem 5.1. Assume that $\kappa_{1} \kappa_{2}<1$, and suppose that insurer $k$, for $k=1,2$ has an exponential utility function (17) and the relative surplus process $\left\{\hat{X}_{k}^{\pi_{k}^{\mathrm{n}}}(t)\right\}_{t \geq 0}$ in (8). Then, for each $\mathfrak{n} \in \mathbb{N}$ and $\mathfrak{a} \geq 1$, the solution to the coupled PDE system in (16) is $V^{k, \mathfrak{n}}(t, x, l)$, for $k=1,2$, which admits the following explicit form:

$$
V^{k, \mathfrak{n}}(t, x, l)=-\frac{1}{q_{k}} \exp \left\{-q_{k}\left[x \mathrm{e}^{r(T-t)}+f^{k, \mathfrak{n}}(t)\right]-A(t) l-B(t)\right\},
$$

where

$$
\begin{aligned}
f^{k, \mathfrak{n}}(t)= & {\left[\frac{\left(\lambda_{k}+\lambda\right)\left(\eta_{k}-\theta_{k}\right)}{r} \bar{\mu}_{k}-\frac{\kappa_{k}\left(\lambda_{j}+\lambda\right)\left(\eta_{j}-\theta_{j}\right)}{r} \bar{\mu}_{j}\right]\left[1-\mathrm{e}^{r(T-t)}\right] } \\
& -\left(\lambda_{k}+\lambda\right)\left(\mathfrak{n}^{\mathfrak{a}} C(\mathfrak{n})+\theta_{k}\right) \int_{t}^{T} \mathrm{e}^{r(T-s)} \mu_{k}\left(a_{k}^{*, \mathfrak{n}}(s)\right) \mathrm{d} s \\
& +\kappa_{k}\left(\lambda_{j}+\lambda\right)\left(\mathfrak{n}^{\mathfrak{a}} C(\mathfrak{n})+\theta_{j}\right) \int_{t}^{T} \mathrm{e}^{r(T-s)} \mu_{j}\left(a_{j}^{*, \mathfrak{n}}(s)\right) \mathrm{d} s \\
& +\left(\lambda_{k}+\lambda\right) \mathfrak{n}^{\mathfrak{a}} \int_{t}^{T} \int_{0}^{a_{k}^{*, \mathfrak{n}}(s)} C(\mathfrak{n}) \mathrm{e}^{r(T-s)} \mathrm{e}^{q_{k} C(\mathfrak{n}) y \mathrm{e}^{r(T-s)}} \bar{F}_{k}(y) \mathrm{d} y \mathrm{~d} s
\end{aligned}
$$




$$
\begin{aligned}
& -\left(\lambda_{j}+\lambda\right) \mathfrak{n}^{\mathfrak{a}} \int_{t}^{T} \int_{0}^{a_{j}^{*, \mathfrak{n}}(s)} \kappa_{k} C(\mathfrak{n}) \mathrm{e}^{r(T-s)} \mathrm{e}^{-\kappa_{k} q_{k} C(\mathfrak{n}) y \mathrm{e}^{r(T-s)}} \bar{F}_{j}(y) \mathrm{d} y \mathrm{~d} s \\
& -\lambda \mathfrak{n}^{\mathfrak{a}} \int_{t}^{T}\left[\int_{0}^{a_{k}^{*, \mathfrak{n}}(s)} C(\mathfrak{n}) \mathrm{e}^{r(T-s)} \mathrm{e}^{q_{k} C(\mathfrak{n}) y \mathrm{e}^{r(T-s)}} \bar{F}_{k}(y) \mathrm{d} y\right] \\
& \cdot\left[\int_{0}^{a_{j}^{*, \mathfrak{n}}(s)} \kappa_{k} q_{k} C(\mathfrak{n}) \mathrm{e}^{r(T-s)} \mathrm{e}^{-\kappa_{k} q_{k} C(\mathfrak{n}) y \mathrm{e}^{r(T-s)}} \bar{F}_{j}(y) \mathrm{d} y\right] \mathrm{d} s \\
& A(t)= \begin{cases}\frac{v_{+} v_{-}-v_{+} v_{-} \mathrm{e}^{-\frac{1}{2}\left(1-\rho_{S}^{2}\right) \sigma^{2}\left(v_{+}-v_{-}\right)(T-t)}}{v_{-}-v_{+} \mathrm{e}^{-\frac{1}{2}\left(1-\rho_{S}^{2}\right) \sigma^{2}\left(v_{+}-v_{-}\right)(T-t)},} & \rho_{S} \neq \pm 1, \\
\frac{m^{2}}{2(\alpha+m \sigma)}\left[1-\mathrm{e}^{-(\alpha+m \sigma)(T-t)}\right], & \rho_{S}=1, \\
\frac{m^{2}}{2(\alpha-m \sigma)}\left[1-\mathrm{e}^{-(\alpha-m \sigma)(T-t)}\right], & \rho_{S}=-1, \text { and } \alpha \neq m \sigma, \\
\frac{m^{2}}{2}(T-t), & \rho_{S}=-1, \text { and } \alpha=m \sigma,\end{cases} \\
& B(t)=\alpha \delta \int_{t}^{T} A(s) \mathrm{d} s
\end{aligned}
$$

with

$$
v_{+,-}=\frac{-\alpha-\rho_{S} \sigma m \pm \sqrt{\alpha^{2}+2 \alpha \rho_{S} \sigma m+\sigma^{2} m^{2}}}{\left(1-\rho_{S}^{2}\right) \sigma^{2}} .
$$

For $0 \leq t \leq T$, let $\left(\hat{a}_{1}^{\mathfrak{n}}, \hat{a}_{2}^{\mathfrak{n}}\right)$, if exists, be the unique solution of the following system

$$
\left\{\begin{array}{l}
\hat{a}_{1}^{\mathfrak{n}}(t)=\frac{\mathrm{e}^{-r(T-t)}}{q_{1} C(\mathfrak{n})} \ln \left\{\frac{\left(\lambda_{1}+\lambda\right)\left(1+\frac{\theta_{1}}{\mathfrak{n}^{\mathfrak{a}} C(\mathfrak{n})}\right)}{\lambda_{1}+\lambda\left(1+h_{1}^{\mathfrak{n}}\left(\hat{a}_{2}^{\mathfrak{n}}(t)\right)\right.}\right\}, \\
\hat{a}_{2}^{\mathfrak{n}}(t)=\frac{\mathrm{e}^{-r(T-t)}}{q_{2} C(\mathfrak{n})} \ln \left\{\frac{\left(\lambda_{2}+\lambda\right)\left(1+\frac{\theta_{2}}{\mathfrak{n}^{\mathfrak{a}} C(\mathfrak{n})}\right)}{\lambda_{2}+\lambda\left(1+h_{2}^{\mathfrak{n}}\left(\hat{a}_{1}^{\mathfrak{n}}(t)\right)\right)}\right\}
\end{array}\right.
$$

then the reinsurance retention levels $\left(a_{1}^{*, \mathfrak{n}}, a_{2}^{*, \mathfrak{n}}\right)$ at equilibrium admit one of the following forms, for $k \neq j \in\{1,2\}$,

1. If $\hat{a}_{k}^{\mathfrak{n}}(t) \leq D_{k}$ and $\hat{a}_{j}^{\mathfrak{n}}(t) \leq D_{j}$, then $\left(a_{k}^{*, \mathfrak{n}}(t), a_{j}^{*, \mathfrak{n}}(t)\right)=\left(\hat{a}_{k}^{\mathfrak{n}}(t), \hat{a}_{j}^{\mathfrak{n}}(t)\right)$;

2. If $\hat{a}_{k}^{\mathfrak{n}}(t)>D_{k}$ and $\hat{a}_{j}^{\mathfrak{n}}(t) \leq D_{j}$, then $\left(a_{k}^{*, \mathfrak{n}}(t), a_{j}^{*, \mathfrak{n}}(t)\right)=\left(D_{k}, \frac{\mathrm{e}^{-r(T-t)}}{q_{j} C(\mathfrak{n})} \ln \left\{\frac{\left(\lambda_{j}+\lambda\right)\left(1+\frac{\theta_{j}}{\mathfrak{n}^{\mathfrak{a} C} C(\mathfrak{n})}\right)}{\lambda_{j}+\lambda\left(1+h_{j}^{\mathfrak{n}}\left(D_{k}\right)\right)}\right\} \wedge D_{j}\right)$;

3. If $\hat{a}_{k}^{\mathfrak{n}}(t)>D_{k}$ and $\hat{a}_{j}^{\mathfrak{n}}(t)>D_{j}$, then $\left(a_{k}^{*, \mathfrak{n}}(t), a_{j}^{*, \mathfrak{n}}(t)\right)=\left(D_{k}, D_{j}\right)$;

and the investment amount $\left(b_{1}^{*, \mathfrak{n}}, b_{2}^{*, \mathfrak{n}}\right)$ at equilibrium is

$$
\left\{\begin{array}{c}
b_{1}^{*, \mathfrak{n}}(t)=\frac{\mathrm{e}^{-r(T-t)}}{1-\kappa_{1} \kappa_{2}}\left[\left(\frac{1}{q_{1}}+\frac{\kappa_{1}}{q_{2}}\right)\left(m-\rho_{S} \sigma A(t)\right)\right], \\
b_{2}^{*, \mathfrak{n}}(t)=\frac{\mathrm{e}^{-r(T-t)}}{1-\kappa_{1} \kappa_{2}}\left[\left(\frac{1}{q_{2}}+\frac{\kappa_{2}}{q_{1}}\right)\left(m-\rho_{S} \sigma A(t)\right)\right] .
\end{array}\right.
$$

Remark 5.2. A sufficient condition under which the equilibrium reinsurance strategies $\left(\hat{a}_{1}^{\mathfrak{n}}, \hat{a}_{2}^{\mathfrak{n}}\right)$ uniquely exist is given in Lemma 5.4 . 
Proof. For the sake of transparent exposition, we shall suppress the arguments of the functions. Consider the following Ansatz:

$$
W^{k, \mathfrak{n}}(t, x, l) \triangleq-\frac{1}{q_{k}} \exp \left\{-q_{k}\left[x \mathrm{e}^{r(T-t)}+f^{k, \mathfrak{n}}(t)+g^{k}(t, l)\right]\right\}
$$

with $f^{k}(T)=0, g^{k}(T, l)=0$, for all $l \in \mathbb{R}$. We shall verify that $V^{k, \mathfrak{n}}=W^{k, \mathfrak{n}}$ in Appendix A. Since we have the following expressions:

(1) $W_{t}^{k, \mathfrak{n}}=\left(r q_{k} x \mathrm{e}^{r(T-t)}-q_{k} f_{t}^{k, \mathfrak{n}}-q_{k} g_{t}^{k}\right) W^{k, \mathfrak{n}}, \quad W_{x}^{k, \mathfrak{n}}=-q_{k} \mathrm{e}^{r(T-t)} W^{k, \mathfrak{n}}, \quad W_{x x}^{k, \mathfrak{n}}=q_{k}^{2} \mathrm{e}^{2 r(T-t)} W^{k, \mathfrak{n}}$,

(2) $W_{l}^{k, \mathfrak{n}}=-q_{k} g_{l}^{k} W^{k, \mathfrak{n}}, \quad W_{l l}^{k, \mathfrak{n}}=-q_{k} g_{l l}^{k} W^{k, \mathfrak{n}}+q_{k}^{2}\left(g_{l}^{k}\right)^{2} W^{k, \mathfrak{n}}, \quad W_{x l}^{k, \mathfrak{n}}=q_{k}^{2} \mathrm{e}^{r(T-t)} g_{l}^{k} W^{k, \mathfrak{n}}$,

(3) $\mathbb{E}\left[W^{k, \mathfrak{n}}\left(t, x-C(\mathfrak{n})\left(Z^{k} \wedge a_{k}(t)\right), l\right)-W^{k, \mathfrak{n}}(t, x, l)\right]$

$$
=\left\{\int_{0}^{a_{k}^{\mathfrak{n}}(t)} q_{k} \mathrm{e}^{r(T-t)} C(\mathfrak{n}) \mathrm{e}^{q_{k} \mathrm{e}^{r(T-t)} C(\mathfrak{n}) y} \bar{F}_{k}(y) \mathrm{d} y\right\} W^{k, \mathfrak{n}},
$$

(4) $\mathbb{E}\left[W^{k, \mathfrak{n}}\left(t, x+\kappa_{k} C(\mathfrak{n})\left(Z^{j} \wedge \hat{a}_{j}^{\mathfrak{n}}(t)\right), l\right)-W^{k, \mathfrak{n}}(t, x, l)\right]$

$$
=-\left\{\int_{0}^{a_{j}^{*, \mathfrak{n}}(t)} q_{k} \kappa_{k} \mathrm{e}^{r(T-t)} C(\mathfrak{n}) \mathrm{e}^{-q_{k} \kappa_{k} \mathrm{e}^{r(T-t)} C(\mathfrak{n}) y} \bar{F}_{j}(y) \mathrm{d} y\right\} W^{k, \mathfrak{n}}
$$

(5) $\mathbb{E}\left[W^{k, \mathfrak{n}}\left(t, x-C(\mathfrak{n})\left(Z^{k} \wedge a_{k}^{\mathfrak{n}}(t)\right)+\kappa_{k} C(\mathfrak{n})\left(Z^{j} \wedge \hat{a}_{j}^{\mathfrak{n}}(t)\right), l\right)-W^{k, \mathfrak{n}}(t, x, l)\right]$

$$
\begin{aligned}
& =\left\{\left[\int_{0}^{a_{k}^{\mathfrak{n}}(t)} q_{k} \mathrm{e}^{r(T-t)} C(\mathfrak{n}) \mathrm{e}^{q_{k} \mathrm{e}^{r(T-t)} C(\mathfrak{n}) y} \bar{F}_{k}(y) \mathrm{d} y+1\right]\right. \\
& \left.\cdot\left[1-\int_{0}^{\hat{a}_{j}^{\mathfrak{n}}(t)} \kappa_{k} q_{k} \mathrm{e}^{r(T-t)} C(\mathfrak{n}) \mathrm{e}^{-\kappa_{k} q_{k} \mathrm{e}^{r(T-t)} C(\mathfrak{n}) y} \bar{F}_{j}(y) \mathrm{d} y\right]-1\right\} W^{k, \mathfrak{n}},
\end{aligned}
$$

for $k, j=1,2$, with $k \neq j$, it implies that ${ }^{2}$ minimizer $a_{k}^{\mathfrak{n}}(t)$ in (13) satisfies

$$
\begin{aligned}
0= & -\left(\lambda_{k}+\lambda\right)\left(\theta_{k}+\mathfrak{n}^{\mathfrak{a}} C(\mathfrak{n})\right) \bar{F}_{k}\left(a_{k}^{\mathfrak{n}}\right) q_{k} \mathrm{e}^{r(T-t)} W^{k, \mathfrak{n}}(t, x, l) \\
& +\mathfrak{n}^{\mathfrak{a}} \lambda_{k} q_{k} \mathrm{e}^{r(T-t)} C(\mathfrak{n}) \exp \left(q_{k} \mathrm{e}^{r(T-t)} C(\mathfrak{n}) a_{k}^{\mathfrak{n}}\right) \bar{F}_{k}\left(a_{k}^{\mathfrak{n}}\right) W^{k, \mathfrak{n}}(t, x, l) \\
& +\mathfrak{n}^{\mathfrak{a}} \lambda q_{k} \mathrm{e}^{r(T-t)} C(\mathfrak{n}) \exp \left(q_{k} \mathrm{e}^{r(T-t)} C(\mathfrak{n}) a_{k}^{\mathfrak{n}}\right) \bar{F}_{k}\left(a_{k}^{\mathfrak{n}}\right)\left[1+h_{k}^{\mathfrak{n}}\left(\hat{a}_{j}^{\mathfrak{n}}\right)\right] W^{k, \mathfrak{n}}(t, x, l) \\
= & -\left(\lambda_{k}+\lambda\right)\left(\theta_{k}+\mathfrak{n}^{\mathfrak{a}} C(\mathfrak{n})\right)+\mathfrak{n}^{\mathfrak{a}} C(\mathfrak{n}) \lambda_{k} \exp \left(q_{k} \mathrm{e}^{r(T-t)} C(\mathfrak{n}) a_{k}^{\mathfrak{n}}\right) \\
& +\mathfrak{n}^{\mathfrak{a}} C(\mathfrak{n}) \lambda \exp \left(q_{k} \mathrm{e}^{r(T-t)} C(\mathfrak{n}) a_{k}^{\mathfrak{n}}\right)\left[1+h_{k}^{\mathfrak{n}}\left(\hat{a}_{j}^{\mathfrak{n}}\right)\right],
\end{aligned}
$$

i.e.

$$
\hat{a}_{k}^{\mathfrak{n}}=\frac{\mathrm{e}^{-r(T-t)}}{q_{k} C(\mathfrak{n})} \ln \left\{\frac{\left(\lambda_{k}+\lambda\right)\left(1+\frac{\theta_{k}}{C(\mathfrak{n}) \mathfrak{n}^{\mathfrak{a}}}\right)}{\left(\lambda_{k}+\lambda\left[1+h_{k}^{\mathfrak{n}}\left(\hat{a}_{j}^{\mathfrak{n}}\right)\right]\right)}\right\} .
$$

\footnotetext{
${ }^{2}$ To be more rigorous, we can first assume that $a_{k} \in\left[0, D_{k}\right)$, where $D_{k} \triangleq \sup \left\{z: F_{k}(z)<1\right\}<\infty$. Then, the possibility that $\bar{F}_{k}\left(a_{k}\right)=0$ can then be excluded and that (26) remains in force for $a_{k}^{\mathfrak{n}} \in\left[0, D_{k}\right)$. Following this argument, we then arrive with Lemma 5.3 that, for each $\mathfrak{n} \in \mathbb{N}$ and $\mathfrak{a} \geq 1, \hat{a}_{k}^{\mathfrak{n}}$ is uniformly bounded, in which the upper bound is independent of $D_{k}$. As the upper bound of $\hat{a}_{k}^{\mathfrak{n}}$ is independent of $D_{k}$, Lemma 5.3 remains valid, and therefore the existence of equilibrium reinsurance strategies $\hat{a}_{k}^{\mathfrak{n}}$, for $k=1,2$, are guaranteed by Lemma 5.4 , even when we let $D_{k}$ approach to $\infty$.
} 
The unique existence of the reinsurance pair $\left(\hat{a}_{1}^{\mathfrak{n}}, \hat{a}_{2}^{\mathfrak{n}}\right)$ is equivalent to the existence of solution to non-linear integral-typed system in (23), which will be established in Lemma 5.4; in accordance of this result, the desired existence of the equilibrium reinsurance strategies $\left(a_{1}^{*, \mathfrak{n}}, a_{2}^{*, \mathfrak{n}}\right)$ follows.

On other hand, the minimizer $b_{k}^{*, \mathfrak{n}}$ in $(14)$ is

$$
b_{k}^{*, \mathfrak{n}}(t)=\frac{m}{q_{k}} \mathrm{e}^{-r(T-t)}+\kappa_{k} b_{j}^{*, \mathfrak{n}}(t)-\rho_{S} \sigma \mathrm{e}^{-r(T-t)} g_{l}^{k},
$$

yielding (24), where $A(t)$ shall be determined below.

Applying (11) to $W^{k, \mathfrak{n}}$ in (25) with (26), and (27) yields

$$
\begin{aligned}
0= & f_{t}^{k, \mathfrak{n}}+g_{t}^{k}+\left(\lambda_{k}+\lambda\right) \mathrm{e}^{r(T-t)}\left[\left(C(\mathfrak{n}) \mathfrak{n}^{\mathfrak{a}}+\theta_{k}\right) \mu_{k}\left(a_{k}^{*, \mathfrak{n}}(t)\right)+\left(\eta_{k}-\theta_{k}\right) \bar{\mu}_{k}\right] \\
& -\kappa_{k}\left(\lambda_{j}+\lambda\right) \mathrm{e}^{r(T-t)}\left[\left(C(\mathfrak{n}) \mathfrak{n}^{\mathfrak{a}}+\theta_{j}\right) \mu_{j}\left(a_{j}^{*, \mathfrak{n}}(t)\right)+\left(\eta_{j}-\theta_{j}\right) \bar{\mu}_{j}\right]+\frac{m^{2} l}{2 q_{k}}+\frac{1}{2} \rho_{S}^{2} \sigma^{2} q_{k} l\left(g_{l}^{k}\right)^{2} \\
& +\alpha(\delta-l) g_{l}^{k}-\frac{1}{2} \sigma^{2} q_{k} l\left(g_{l}^{k}\right)^{2}-\rho_{S} \sigma m l g_{l}^{k}+\frac{1}{2} \sigma^{2} l g_{l l}^{k} \\
& -\left(\lambda_{k}+\lambda\right) \mathfrak{n}^{\mathfrak{a}} \int_{0}^{a_{k}^{*}(t)} \mathrm{e}^{r(T-t)} C(\mathfrak{n}) \mathrm{e}^{q_{k} \mathrm{e}^{r(T-t)} C(\mathfrak{n}) y} \bar{F}_{k}(y) \mathrm{d} y \\
& +\left(\lambda_{j}+\lambda\right) \mathfrak{n}^{\mathfrak{a}} \int_{0}^{a_{j}^{*, \mathfrak{n}}(t)} \kappa_{k} \mathrm{e}^{r(T-t)} C(\mathfrak{n}) \mathrm{e}^{-\kappa_{k} q_{k} \mathrm{e}^{r(T-t)} C(\mathfrak{n}) y} \bar{F}_{j}(y) \mathrm{d} y \\
& +\lambda \mathfrak{n}^{\mathfrak{a}}\left[\int_{0}^{a_{k}^{*, \mathfrak{n}}(t)} \mathrm{e}^{r(T-t)} C(\mathfrak{n}) \mathrm{e}^{q_{k} \mathrm{e}^{r(T-t)} C(\mathfrak{n}) y} \bar{F}_{k}(y) \mathrm{d} y\right] \\
& \cdot\left[\int_{0}^{a_{j}^{*, \mathfrak{n}}(t)} \kappa_{k} q_{k} \mathrm{e}^{r(T-t)} C(\mathfrak{n}) \mathrm{e}^{-\kappa_{k} q_{k} \mathrm{e}^{r(T-t)} C(\mathfrak{n}) y} \bar{F}_{j}(y) \mathrm{d} y\right]
\end{aligned}
$$

for $j=1,2$ with $j \neq k$, which can be decomposed into two equations

$$
0=g_{t}^{k}+\frac{m^{2} l}{2 q_{k}}-\frac{1}{2}\left(1-\rho_{S}^{2}\right) \sigma^{2} q_{k} l\left(g_{l}^{k}\right)^{2}+\alpha \delta g_{l}^{k}-\left(\alpha+\rho_{S} \sigma m\right) l g_{l}^{k}+\frac{1}{2} \sigma^{2} l g_{l l}^{k}
$$

and

$$
\begin{aligned}
0= & f_{t}^{k, \mathfrak{n}}+\left(\lambda_{k}+\lambda\right) \mathrm{e}^{r(T-t)}\left[\left(C(\mathfrak{n}) \mathfrak{n}^{\mathfrak{a}}+\theta_{k}\right) \mu_{k}\left(a_{k}^{*, \mathfrak{n}}(t)\right)+\left(\eta_{k}-\theta_{k}\right) \bar{\mu}_{k}\right] \\
& -\kappa_{k}\left(\lambda_{j}+\lambda\right) \mathrm{e}^{r(T-t)}\left[\left(C(\mathfrak{n}) \mathfrak{n}^{\mathfrak{a}}+\theta_{j}\right) \mu_{j}\left(a_{j}^{*, \mathfrak{n}}(t)\right)+\left(\eta_{j}-\theta_{j}\right) \bar{\mu}_{j}\right] \\
& -\left(\lambda_{k}+\lambda\right) \mathfrak{n}^{\mathfrak{a}} \int_{0}^{a_{k}^{*, \mathfrak{n}}(t)} \mathrm{e}^{r(T-t)} C(\mathfrak{n}) \mathrm{e}^{q_{k} \mathrm{e}^{r(T-t)} C(\mathfrak{n}) y} \bar{F}_{k}(y) \mathrm{d} y \\
& +\left(\lambda_{j}+\lambda\right) \mathfrak{n}^{\mathfrak{a}} \int_{0}^{a_{j}^{*, \mathfrak{n}}(t)} \kappa_{k} \mathrm{e}^{r(T-t)} C(\mathfrak{n}) \mathrm{e}^{-\kappa_{k} q_{k} \mathrm{e}^{r(T-t)} C(\mathfrak{n}) y} \bar{F}_{j}(y) \mathrm{d} y \\
& +\lambda \mathfrak{n}^{\mathfrak{a}}\left[\int_{0}^{a_{k}^{*, \mathfrak{n}}(t)} \mathrm{e}^{r(T-t)} C(\mathfrak{n}) \mathrm{e}^{q_{k} \mathrm{e}^{r(T-t)} C(\mathfrak{n}) y} \bar{F}_{k}(y) \mathrm{d} y\right] \\
& \cdot\left[\int_{0}^{a_{j}^{*, \mathfrak{n}}(t)} \kappa_{k} q_{k} \mathrm{e}^{r(T-t)} C(\mathfrak{n}) \mathrm{e}^{-\kappa_{k} q_{k} \mathrm{e}^{r(T-t)} C(\mathfrak{n}) y} \bar{F}_{j}(y) \mathrm{d} y\right]
\end{aligned}
$$


To solve (28), we assume that $g^{k}(t, l)$ admits the following affine form:

$$
g^{k}(t, l)=\frac{1}{q_{k}}(A(t) l+B(t))
$$

when substituting $A$ and $B$ into (28), we have

$$
A_{t} l+B_{t}+\frac{m^{2} l}{2}-\frac{1}{2}\left(1-\rho_{S}^{2}\right) \sigma^{2} l(A(t))^{2}+\alpha(\delta-l) A(t)-\rho_{S} \sigma m l A(t)=0 .
$$

Matching coefficients yields

$$
\left\{\begin{array}{l}
A_{t}-\frac{1}{2}\left(1-\rho_{S}^{2}\right) \sigma^{2}(A(t))^{2}-\left(\alpha+\rho_{S} \sigma m\right) A(t)+\frac{m^{2}}{2}=0 \\
A(T)=0
\end{array}\right.
$$

and

$$
\left\{\begin{array}{l}
B_{t}+\alpha \delta A(t)=0 \\
B(T)=0
\end{array}\right.
$$

in which the solutions, $A$ and $B$, admit the forms in (21) and (22), respectively.

Finally, from (29), it is clear that $f^{k, \mathfrak{n}}$ is a solution of the linear ordinary differential equations, which admits the form in (20).

We now proceed on proving the existence of the solution to the system in (23). First, we show that $\hat{a}_{k}^{\mathfrak{n}}(t), k=1,2$ is uniformly bounded, for $t \in[0, T]$, in the following lemma.

Lemma 5.3. Let $\hat{a}_{k}^{\mathfrak{n}}(t)$ defined in (23), for $k=1,2$. Then, for each $\mathfrak{n} \in \mathbb{N}$ and $\mathfrak{a} \geq 1$,

$$
\frac{\mathrm{e}^{-r(T-t)}}{q_{k} C(\mathfrak{n})} \ln \left(1+\frac{\theta_{k}}{C(\mathfrak{n}) \mathfrak{n}^{\mathfrak{a}}}\right) \leq \hat{a}_{k}^{\mathfrak{n}}(t) \leq \frac{\mathrm{e}^{-r(T-t)}}{q_{k} C(\mathfrak{n})} \ln \left[\frac{\lambda_{k}+\lambda}{\lambda_{k}}\left(1+\frac{\theta_{k}}{C(\mathfrak{n}) \mathfrak{n}^{\mathfrak{a}}}\right)\right] .
$$

Proof. Note that

$$
\frac{\partial h_{k}^{\mathfrak{n}}}{\partial \hat{a}_{j}^{\mathfrak{n}}}=-\kappa_{k} q_{k} \mathrm{e}^{r(T-t)} C(\mathfrak{n}) \mathrm{e}^{-\kappa_{k} q_{k} \mathrm{e}^{r(T-t)} C(\mathfrak{n}) \hat{a}_{j}^{\mathfrak{n}}(t)} \bar{F}_{j}\left(\hat{a}_{j}^{\mathfrak{n}}(t)\right)<0 .
$$

Thus $h_{k}\left(\hat{a}_{j}^{\mathfrak{n}}\right)$ decreases in $\hat{a}_{j}^{\mathfrak{n}}$, and hence

$$
-\int_{0}^{+\infty} \kappa_{k} q_{k} \mathrm{e}^{r(T-t)} C(\mathfrak{n}) \mathrm{e}^{-\kappa_{k} q_{k} \mathrm{e}^{r(T-t)} C(\mathfrak{n}) y} \bar{F}_{j}(y) \mathrm{d} y \leq h_{k}^{\mathfrak{n}}\left(\hat{a}_{j}^{\mathfrak{n}}\right) \leq 0
$$

Furthermore, $0 \leq F_{j}(y) \leq 1$ leads to

$$
-\int_{0}^{+\infty} \kappa_{k} q_{k} \mathrm{e}^{r(T-t)} C(\mathfrak{n}) \mathrm{e}^{-\kappa_{k} q_{k} \mathrm{e}^{r(T-t)} C(\mathfrak{n}) y} \bar{F}_{j}(y) \mathrm{d} y \geq-\int_{0}^{+\infty} \kappa_{k} q_{k} \mathrm{e}^{r(T-t)} C(\mathfrak{n}) \mathrm{e}^{-\kappa_{k} q_{k} \mathrm{e}^{r(T-t)} C(\mathfrak{n}) y} \mathrm{~d} y=-1 .
$$

Hence $-1 \leq h_{k}^{\mathfrak{n}}\left(\hat{a}_{j}^{\mathfrak{n}}\right) \leq 0$. Then it follows that

$$
\frac{\mathrm{e}^{-r(T-t)}}{q_{k} C(\mathfrak{n})} \ln \left(1+\frac{\theta_{k}}{C(\mathfrak{n}) \mathfrak{n}^{\mathfrak{a}}}\right) \leq \hat{a}_{k}^{\mathfrak{n}}(t) \leq \frac{\mathrm{e}^{-r(T-t)}}{q_{k} C(\mathfrak{n})} \ln \left[\frac{\lambda_{k}+\lambda}{\lambda_{k}}\left(1+\frac{\theta_{k}}{C(\mathfrak{n}) \mathfrak{n}^{\mathfrak{a}}}\right)\right]
$$

immediately. 
Next lemma provides the sufficient condition for the unique existence of the couple system of non-linear integral-typed equations in (23).

Lemma 5.4. If

$$
\kappa_{1} \kappa_{2}\left(\frac{\lambda\left(\lambda_{1}+\lambda\right)\left(1+\frac{\theta_{1}}{C(\mathfrak{n}) \mathfrak{n}^{\mathfrak{a}}}\right)}{\lambda_{1}^{2}}\right)\left(\frac{\lambda\left(\lambda_{2}+\lambda\right)\left(1+\frac{\theta_{2}}{C(\mathfrak{n}) \mathfrak{n}^{\mathfrak{a}}}\right)}{\lambda_{2}^{2}}\right)<1,
$$

the coupled system of non-linear integral-typed equations (23) admits a unique solution $\left(\hat{a}_{1}^{\mathfrak{n}}, \hat{a}_{2}^{\mathfrak{n}}\right)$, where $\hat{a}_{1}^{\mathfrak{n}}$ and $\hat{a}_{2}^{\mathfrak{n}} \in C[0, T]$.

Remark 5.5. Note that condition (31) is rather mild as it is satisfied when the intensity of the systematic jump risk $\lambda$ is small relative to the intensities of the idiosyncratic jump risks $\lambda_{k}$, for $k=1,2$. This is in line with reality as insurers choose their reinsurance-investment strategies to diversify their risks, and therefore the magnitude of the systematic risk in their surpluses should be small relative to their idiosyncratic risks.

Proof. Consider the following sequence $\left\{\hat{a}_{k, l}^{\mathfrak{n}}\right\}_{l=0}^{\infty}$, for $k=1,2$, where

$$
\left\{\begin{array}{l}
\hat{a}_{1,0}^{\mathfrak{n}}(t) \triangleq \frac{\mathrm{e}^{-r(T-t)}}{q_{1} C(\mathfrak{n})} \ln \left\{1+\frac{\theta_{k}}{C(\mathfrak{n}) \mathfrak{n}^{\mathfrak{a}}}\right\}, \\
\hat{a}_{2,0}^{\mathfrak{n}}(t) \triangleq \frac{\mathrm{e}^{-r(T-t)}}{q_{2} C(\mathfrak{n})} \ln \left\{\frac{1}{\mathfrak{A}_{2}^{\mathfrak{n}}+\mathfrak{B}_{2}^{\mathfrak{n}}\left[h_{2}^{\mathfrak{n}}\left(\hat{a}_{1,0}^{\mathfrak{n}}(t)\right)+1\right]}\right\} \\
\hat{a}_{1, l}^{\mathfrak{n}}(t) \triangleq \frac{\mathrm{e}^{-r(T-t)}}{q_{1} C(\mathfrak{n})} \ln \left\{\frac{1}{\mathfrak{A}_{1}^{\mathfrak{n}}+\mathfrak{B}_{1}^{\mathfrak{n}}\left[h_{1}^{\mathfrak{n}}\left(\hat{a}_{2, l-1}^{\mathfrak{n}}(t)\right)+1\right]}\right\}, \quad \text { for } \quad l \geq 1, \\
\hat{a}_{2, l}^{\mathfrak{n}}(t) \triangleq \frac{\mathrm{e}^{-r(T-t)}}{q_{2} C(\mathfrak{n})} \ln \left\{\frac{1}{\mathfrak{A}_{2}^{\mathfrak{n}}+\mathfrak{B}_{2}^{\mathfrak{n}}\left[h_{2}^{\mathfrak{n}}\left(\hat{a}_{1, l}^{\mathfrak{n}}(t)\right)+1\right]}\right\}, \quad \text { for } \quad l \geq 1,
\end{array}\right.
$$

where

$$
\mathfrak{A}_{k}^{\mathfrak{n}} \triangleq \frac{\lambda_{k}}{\left(\lambda_{k}+\lambda\right)\left(1+\frac{\theta_{k}}{C(\mathfrak{n}) \mathfrak{n}^{\mathfrak{a}}}\right)}, \quad \mathfrak{B}_{k}^{\mathfrak{n}} \triangleq \frac{\lambda}{\left(\lambda_{k}+\lambda\right)\left(1+\frac{\theta_{k}}{C(\mathfrak{n}) \mathfrak{n}^{\mathfrak{a}}}\right)}
$$

for $k=1,2$. It suffices to show that $\left\{\hat{a}_{k, l}^{\mathfrak{n}}\right\}_{l=0}^{\infty}$ is a Cauchy sequence with respect to the uniform norm.

Firstly, the uniform boundedness of the sequence $\left\{\hat{a}_{1, l}^{\mathfrak{n}}\right\}$ is established via Lemma 5.3, which readily implies that, for each $\mathfrak{n} \in \mathbb{N}$ and $\mathfrak{a} \geq 1$,

$$
0<\mathfrak{A}_{k}^{\mathfrak{n}} \leq \mathfrak{A}_{k}^{\mathfrak{n}}+\mathfrak{B}_{k}^{\mathfrak{n}}\left[h_{k}^{\mathfrak{n}}\left(\hat{a}_{j, l}^{\mathfrak{n}}(t)\right)+1\right] \leq \mathfrak{A}_{k}^{\mathfrak{n}}+\mathfrak{B}_{k}^{\mathfrak{n}}=\frac{1}{1+\frac{\theta_{k}}{C(\mathfrak{n}) \mathfrak{n}^{\mathfrak{a}}}}<1
$$

and so by definition,

$$
\frac{1}{q_{k} C(\mathfrak{n})} \ln \left(1+\frac{\theta_{k}}{C(\mathfrak{n}) \mathfrak{n}^{\mathfrak{a}}}\right) \leq \hat{a}_{k, l}^{\mathfrak{n}}(t) \leq \frac{1}{q_{k} C(\mathfrak{n})} \ln \left[\frac{\lambda_{k}+\lambda}{\lambda_{k}}\left(1+\frac{\theta_{k}}{C(\mathfrak{n}) \mathfrak{n}^{\mathfrak{a}}}\right)\right],
$$

for all $k=1,2, l \in \mathbb{N}$, and $t \in[0, T]$. 
Note that

$$
\begin{aligned}
& \left|\hat{a}_{1, l+1}^{\mathfrak{n}}(t)-\hat{a}_{1, l}^{\mathfrak{n}}(t)\right| \leq \frac{\mathrm{e}^{-r(T-t)}}{q_{1} C(\mathfrak{n})} \mid \ln \left\{\frac{1}{\mathfrak{A}_{1}^{\mathfrak{n}}+\mathfrak{B}_{1}^{\mathfrak{n}}\left[h_{1}^{\mathfrak{n}}\left(\hat{a}_{2, l}^{\mathfrak{n}}(t)\right)+1\right]}\right\} \\
& -\ln \left\{\frac{1}{\mathfrak{A}_{1}^{\mathfrak{n}}+\mathfrak{B}_{1}^{\mathfrak{n}}\left[h_{1}^{\mathfrak{n}}\left(\hat{a}_{2, l-1}^{\mathfrak{n}}(t)\right)+1\right]}\right\} \mid \\
& \leq \frac{\mathrm{e}^{-r(T-t)}}{q_{1} C(\mathfrak{n})}\left|\frac{1}{\mathfrak{A}_{1}^{\mathfrak{n}}+\mathfrak{B}_{1}^{\mathfrak{n}}\left[h_{1}^{\mathfrak{n}}\left(\hat{a}_{2, l}^{\mathfrak{n}}(t)\right)+1\right]}-\frac{1}{\mathfrak{A}_{1}^{\mathfrak{n}}+\mathfrak{B}_{1}^{\mathfrak{n}}\left[h_{1}^{\mathfrak{n}}\left(\hat{a}_{2, l-1}^{\mathfrak{n}}(t)\right)+1\right]}\right| \\
& =\frac{\mathrm{e}^{-r(T-t)}}{q_{1} C(\mathfrak{n})} \frac{\mathfrak{B}_{1}^{\mathfrak{n}}\left|h_{1}^{\mathfrak{n}}\left(\hat{a}_{2, l}^{\mathfrak{n}}(t)\right)-h_{1}^{\mathfrak{n}}\left(\hat{a}_{2, l-1}^{\mathfrak{n}}(t)\right)\right|}{\left[\mathfrak{A}_{1}^{\mathfrak{n}}+\mathfrak{B}_{1}^{\mathfrak{n}}\left[h_{1}^{\mathfrak{n}}\left(\hat{a}_{2, l}^{\mathfrak{n}}(t)\right)+1\right]\right]\left[\mathfrak{A}_{1}^{\mathfrak{n}}+\mathfrak{B}_{1}^{\mathfrak{n}}\left[h_{1}^{\mathfrak{n}}\left(\hat{a}_{2, l-1}^{\mathfrak{n}}(t)\right)+1\right]\right]} \\
& =\frac{\mathrm{e}^{-r(T-t)}}{q_{1} C(\mathfrak{n})} \frac{\mathfrak{B}_{1}^{\mathfrak{n}}}{\left[\mathfrak{A}_{1}^{\mathfrak{n}}+\mathfrak{B}_{1}^{\mathfrak{n}}\left[h_{1}^{\mathfrak{n}}\left(\hat{a}_{2, l}^{\mathfrak{n}}(t)\right)+1\right]\right]\left[\mathfrak{A}_{1}^{\mathfrak{n}}+\mathfrak{B}_{1}^{\mathfrak{n}}\left[h_{1}^{\mathfrak{n}}\left(\hat{a}_{2, l-1}^{\mathfrak{n}}(t)\right)+1\right]\right]} \\
& \cdot\left|\int_{\hat{a}_{2, l-1}^{\mathrm{n}}(t)}^{\hat{a}_{2, l}^{\mathrm{n}}(t)} C(\mathfrak{n}) \kappa_{1} q_{1} \mathrm{e}^{r(T-t)} \mathrm{e}^{-\kappa_{1} q_{1} \mathrm{e}^{-r(T-t)} C(\mathfrak{n}) y} \bar{F}_{2}(y) \mathrm{d} y\right| \\
& \leq \frac{\mathrm{e}^{-r(T-t)}}{q_{1} C(\mathfrak{n})} \frac{\mathfrak{B}_{1}^{\mathfrak{n}}}{\left[\mathfrak{A}_{1}^{\mathfrak{n}}+\mathfrak{B}_{1}^{\mathfrak{n}}\left[h_{1}^{\mathfrak{n}}\left(\hat{a}_{2, l}^{\mathfrak{n}}(t)\right)+1\right]\right]\left[\mathfrak{A}_{1}^{\mathfrak{n}}+\mathfrak{B}_{1}^{\mathfrak{n}}\left[h_{1}^{\mathfrak{n}}\left(\hat{a}_{2, l-1}^{\mathfrak{n}}(t)\right)+1\right]\right]} \\
& \cdot\left|\mathrm{e}^{-\kappa_{1} q_{1} \mathrm{e}^{r(T-t)} C(\mathfrak{n}) \hat{a}_{2, l}^{\mathfrak{n}}(t)}-\mathrm{e}^{-\kappa_{1} q_{1} \mathrm{e}^{r(T-t)} C(\mathfrak{n}) \hat{a}_{2, l-1}^{\mathfrak{n}}(t)}\right| \\
& =\frac{\mathrm{e}^{-r(T-t)}}{q_{1} C(\mathfrak{n})} \frac{\mathfrak{B}_{1}^{\mathfrak{n}}}{\left[\mathfrak{A}_{1}^{\mathfrak{n}}+\mathfrak{B}_{1}^{\mathfrak{n}}\left[h_{1}^{\mathfrak{n}}\left(\hat{a}_{2, l}^{\mathfrak{n}}(t)\right)+1\right]\right]\left[\mathfrak{A}_{1}^{\mathfrak{n}}+\mathfrak{B}_{1}^{\mathfrak{n}}\left[h_{1}^{\mathfrak{n}}\left(\hat{a}_{2, l-1}^{\mathfrak{n}}(t)\right)+1\right]\right]} \\
& \cdot\left|\kappa_{1} q_{1} \mathrm{e}^{r(T-t)} C(\mathfrak{n}) \mathrm{e}^{-\kappa_{1} q_{1} \mathrm{e}^{r(T-t)} C(\mathfrak{n}) a_{0}^{\mathfrak{n}}}\right|\left|\hat{a}_{2, l}^{\mathfrak{n}}(t)-\hat{a}_{2, l-1}^{\mathfrak{n}}(t)\right| \\
& =\frac{\kappa_{1} \mathfrak{B}_{1}^{\mathfrak{n}}}{\left[\mathfrak{A}_{1}^{\mathfrak{n}}+\mathfrak{B}_{1}^{\mathfrak{n}}\left[h_{1}^{\mathfrak{n}}\left(\hat{a}_{2, l}^{\mathfrak{n}}(t)\right)+1\right]\right]\left[\mathfrak{A}_{1}^{\mathfrak{n}}+\mathfrak{B}_{1}^{\mathfrak{n}}\left[h_{1}^{\mathfrak{n}}\left(\hat{a}_{2, l-1}^{\mathfrak{n}}(t)\right)+1\right]\right]}\left|\hat{a}_{2, l}^{\mathfrak{n}}(t)-\hat{a}_{2, l-1}^{\mathfrak{n}}(t)\right| \\
& \leq \frac{\kappa_{1} \mathfrak{B}_{1}^{\mathfrak{n}}}{\left(\mathfrak{A}_{1}^{\mathfrak{n}}\right)^{2}}\left|\hat{a}_{2, l}^{\mathfrak{n}}(t)-\hat{a}_{2, l-1}^{\mathfrak{n}}(t)\right|=\frac{\kappa_{1} \lambda\left(\lambda_{1}+\lambda\right)\left(1+\frac{\theta_{1}}{C(\mathfrak{n}) \mathfrak{n}^{\mathfrak{a}}}\right)}{\lambda_{1}^{2}}\left|\hat{a}_{2, l}^{\mathfrak{n}}(t)-\hat{a}_{2, l-1}^{\mathfrak{n}}(t)\right|,
\end{aligned}
$$

where the second inequality follows from (34); the fourth equality follows from the definition of $h_{k}^{\mathfrak{n}}$ in (18), for $k=1,2$; the sixth equality follows from the mean-value theorem that there exists $a_{0}^{\mathfrak{n}} \in\left[\hat{a}_{2, l-1}^{\mathfrak{n}}(t), \hat{a}_{2, l}^{\mathfrak{n}}(t)\right]$ such that

$$
\begin{gathered}
\left|\mathrm{e}^{-\kappa_{1} q_{1} \mathrm{e}^{r(T-t)} C(\mathfrak{n}) \hat{a}_{2, l}^{\mathfrak{n}}(t)}-\mathrm{e}^{-\kappa_{1} q_{1} \mathrm{e}^{r(T-t)} C(\mathfrak{n}) \hat{a}_{2, l-1}^{\mathfrak{n}}(t) \mid=}\right|\left|\kappa_{1} q_{1} \mathrm{e}^{r(T-t)} C(\mathfrak{n}) \mathrm{e}^{-\kappa_{1} q_{1} \mathrm{e}^{r(T-t)} C(\mathfrak{n}) a_{0}^{\mathfrak{n}}}\right| \\
\cdot\left|\hat{a}_{2, l}^{\mathfrak{n}}(t)-\hat{a}_{2, l-1}^{\mathfrak{n}}(t)\right| ;
\end{gathered}
$$


and the eighth inequality follows from the fact that

$$
-1 \leq h_{k}^{\mathfrak{n}}(a) \leq 0
$$

This implies that

$$
\left\|\hat{a}_{1, l+1}^{\mathfrak{n}}-\hat{a}_{1, l}^{\mathfrak{n}}\right\|_{\infty} \leq \frac{\kappa_{1} \lambda\left(\lambda_{1}+\lambda\right)\left(1+\frac{\theta_{1}}{C(\mathfrak{n}) \mathfrak{n}^{\mathfrak{a}}}\right)}{\lambda_{1}^{2}}\left\|\hat{a}_{2, l}^{\mathfrak{n}}-\hat{a}_{2, l-1}^{\mathfrak{n}}\right\|_{\infty},
$$

and by symmetry, we also have

$$
\left\|\hat{a}_{2, l}^{\mathfrak{n}}-\hat{a}_{2, l-1}^{\mathfrak{n}}\right\|_{\infty} \leq \frac{\kappa_{2} \lambda\left(\lambda_{2}+\lambda\right)\left(1+\frac{\theta_{2}}{C(\mathfrak{n}) \mathfrak{n}^{\mathfrak{a}}}\right)}{\lambda_{2}^{2}}\left\|\hat{a}_{1, l}^{\mathfrak{n}}(t)-\hat{a}_{1, l-1}^{\mathfrak{n}}\right\|_{\infty}
$$

which implies that

$$
\begin{aligned}
& \left\|\hat{a}_{1, l+1}^{\mathfrak{n}}-\hat{a}_{1, l}^{\mathfrak{n}}\right\|_{\infty} \\
\leq & \kappa_{1} \kappa_{2}\left(\frac{\lambda\left(\lambda_{1}+\lambda\right)\left(1+\frac{\theta_{1}}{C(\mathfrak{n}) \mathfrak{n}^{\mathfrak{a}}}\right)}{\lambda_{1}^{2}}\right)\left(\frac{\lambda\left(\lambda_{2}+\lambda\right)\left(1+\frac{\theta_{2}}{C(\mathfrak{n}) \mathfrak{n}^{\mathfrak{a}}}\right)}{\lambda_{2}^{2}}\right)\left\|\hat{a}_{1, l}^{\mathfrak{n}}-\hat{a}_{1, l-1}^{\mathfrak{n}}\right\|_{\infty} \\
\leq & {\left[\kappa_{1} \kappa_{2}\left(\frac{\lambda\left(\lambda_{1}+\lambda\right)\left(1+\frac{\theta_{1}}{C(\mathfrak{n}) \mathfrak{n}^{\mathfrak{a}}}\right)}{\lambda_{1}^{2}}\right)\left(\frac{\lambda\left(\lambda_{2}+\lambda\right)\left(1+\frac{\theta_{2}}{C(\mathfrak{n}) \mathfrak{n}^{\mathfrak{a}}}\right)}{\lambda_{2}^{2}}\right)\right]^{l}\left\|\hat{a}_{1,1}^{\mathfrak{n}}-\hat{a}_{1,0}^{\mathfrak{n}}\right\|_{\infty} . }
\end{aligned}
$$

This shows that, given a large integer $n$, for any $m>n$, we have

$$
\begin{aligned}
\left\|\hat{a}_{1, m}^{\mathfrak{n}}(t)-\hat{a}_{1, n}^{\mathfrak{n}}(t)\right\|_{\infty} & \leq \sum_{l=n}^{\infty}\left\|\hat{a}_{1, l+1}^{\mathfrak{n}}(t)-\hat{a}_{1, l}^{\mathfrak{n}}(t)\right\|_{\infty} \\
& \leq\left(\frac{\Upsilon^{n}}{1-\Upsilon}\right)\left\|\hat{a}_{1,1}^{\mathfrak{n}}(t)-\hat{a}_{1,0}^{\mathfrak{n}}(t)\right\|_{\infty},
\end{aligned}
$$

where

$$
\Upsilon \triangleq \kappa_{1} \kappa_{2}\left(\frac{\lambda\left(\lambda_{1}+\lambda\right)\left(1+\frac{\theta_{1}}{C(\mathfrak{n}) \mathfrak{n}^{\mathfrak{a}}}\right)}{\lambda_{1}^{2}}\right)\left(\frac{\lambda\left(\lambda_{2}+\lambda\right)\left(1+\frac{\theta_{2}}{C(\mathfrak{n}) \mathfrak{n}^{\mathfrak{a}}}\right)}{\lambda_{2}^{2}}\right)
$$

Based on the uniform boundedness of sequence $\left\{\hat{a}_{1, l}^{\mathfrak{n}}\right\}$ and the assumption that $\Upsilon<1$, we can henceforth conclude that $\left\{\hat{a}_{1, l}^{\mathfrak{n}}\right\}$ is Cauchy sequence with respect to uniform norm. This means that the left and right-hand sides of system (32) converge uniformly to a limit $\hat{a}_{k}^{\mathfrak{n}}$, for $k=1,2$, when $l$ approaches to $\infty$. To show uniqueness of the limit $\hat{a}_{k}^{\mathfrak{n}}$, for $k=1,2$, assume that there exist more than one limits, i.e. $a_{k}^{\mathfrak{n}}$ and $\hat{a}_{k}^{\mathfrak{n}}$. We can then follow the similar arguments above leading to (36) to arrive with the desired contradiction. 


\section{Nash equilibrium for insurers in diffusion-approximated model: Case of CARA insurers}

Having established the Nash equilibrium for the case of general compound Poisson risk model, we shall consider finding the Nash equilibrium of Problem 3.1 for the diffusion approximated model (see, for example, Rolski et al. (1999)).

Consider the process $\left\{\hat{\mathcal{X}}_{k}^{\pi_{k}}(t)\right\}_{t \geq 0}$, for $\left\{\tilde{\pi}_{k}\right\}_{t \geq 0} \triangleq\left\{\left(\tilde{a}_{k}(t), \tilde{b}_{k}(t)\right)\right\}_{t \geq 0} \in \Pi_{k}$, where

$$
\left\{\begin{aligned}
\mathrm{d} \hat{\mathcal{X}}_{k}^{\tilde{\pi}_{k}}(t)= & \left\{r \hat{\mathcal{X}}_{k}^{\tilde{\pi}_{k}}(t)+\left(\lambda_{k}+\lambda\right)\left[\theta_{k} \mu_{k}\left(\tilde{a}_{k}(t)\right)+\left(\eta_{k}-\theta_{k}\right) \bar{\mu}_{k}\right]\right. \\
& -\kappa_{k}\left(\lambda_{j}+\lambda\right)\left[\theta_{j} \mu_{j}\left(\tilde{a}_{j}(t)\right)+\left(\eta_{j}-\theta_{j}\right) \bar{\mu}_{j}\right] \\
& \left.+m\left[\tilde{b}_{k}(t)-\kappa_{k} \tilde{b}_{j}(t)\right] L(t)\right\} \mathrm{d} t+\left[\tilde{b}_{k}(t)-\kappa_{k} \tilde{b}_{j}(t)\right] \sqrt{L(t)} \mathrm{d} B_{S}(t) \\
& -\gamma_{k} \mathrm{~d} B^{Z^{k}}(t)+\kappa_{k} \gamma_{j} \mathrm{~d} B^{Z^{j}}(t) \\
\hat{\mathcal{X}}_{k}^{\tilde{\pi}_{k}}(0)= & \hat{x}_{k},
\end{aligned}\right.
$$

where $\left\{B^{Z^{k}}\right\}_{t \geq 0}$ denotes the standard Brownian motion and that

$$
\begin{aligned}
\gamma_{k} & \triangleq \sqrt{\left(\lambda+\lambda_{k}\right) \mathbb{E}\left[\left(Z^{k} \wedge \tilde{a}_{k}\right)^{2}\right]} \\
\mathrm{d}\left\langle B^{Z^{1}}, B^{Z^{2}}\right\rangle & =\rho \mathrm{d} t, \text { where } \rho \triangleq \frac{\lambda}{\gamma_{1} \gamma_{2}} \mathbb{E}\left[\left(Z^{1} \wedge \tilde{a}_{1}\right)\right] \mathbb{E}\left[\left(Z^{2} \wedge \tilde{a}_{2}\right)\right],
\end{aligned}
$$

and $\mathrm{d}\left\langle B^{Z^{k}}, B_{S}\right\rangle=0$, for $k \neq j \in\{1,2\}$.

To study the Nash equilibrium between two insurers with the surplus difference process $\left\{\hat{\mathcal{X}}_{k}^{\tilde{\pi}_{k}}(t)\right\}_{t \geq 0}$ in (37), for $k=1,2$, consider the corresponding objective function of insurer $k$, for $k=1,2$, denoted as $\tilde{J}_{k}$, where

$$
\tilde{J}_{k}\left(t, x_{k}, x_{j}, \tilde{\pi}_{k}(t), \tilde{\pi}_{j}(t)\right) \triangleq \mathbb{E}\left[U^{k}\left(\hat{\mathcal{X}}_{k}^{\tilde{\pi}_{k}}(T)\right) \mid \hat{\mathcal{X}}_{k}^{\tilde{\pi}_{k}}(t)=x, L(t)=l\right]
$$

Given the strategy of his competitor $j \in\{1,2\}$, i.e. $\tilde{\pi}_{j}=\left(\tilde{a}_{j}, \tilde{b}_{j}\right)$, the objective function $\tilde{J}_{k}\left(t, x_{k}, x_{j}, \tilde{\pi}_{k}(t), \tilde{\pi}_{j}(t)\right)$ of insurer $k \neq j$ is to find an optimal strategy $\hat{\tilde{\pi}}_{k} \triangleq\left(\hat{\tilde{a}}_{k}, \hat{\tilde{b}}_{k}\right)$ such that

$$
\max _{\tilde{\pi}_{k}=\left(\tilde{a}_{k}, \tilde{b}_{k}\right) \in \Pi_{k}} \tilde{J}_{k}\left(t, x_{k}, x_{j}, \tilde{\pi}_{k}(t), \tilde{\pi}_{j}(t)\right)=\tilde{J}_{k}\left(t, x_{k}, x_{j}, \hat{\tilde{\pi}}_{k}(t), \tilde{\pi}_{j}(t)\right),
$$

Denote $\tilde{V}^{k}$ to be the corresponding value function of insurer $k$, i.e.

$$
\tilde{V}^{k}(t, x, l) \triangleq \sup _{\tilde{\pi}_{k} \in \Pi_{k}} \mathbb{E}\left[U^{k}\left(\hat{\mathcal{X}}_{k}^{\tilde{\pi}_{k}}(T)\right) \mid \hat{\mathcal{X}}_{k}^{\tilde{\pi}_{k}}(t)=x, L(t)=l\right],
$$

and the corresponding Nash equilibrium is

$$
\left\{\begin{array}{l}
\tilde{J}_{1}\left(t, x_{1}, x_{2}, \hat{\tilde{\pi}}_{1}(t), \tilde{\pi}_{2}^{*}(t)\right) \leq \tilde{J}_{1}\left(t, x_{1}, x_{2}, \tilde{\pi}_{1}^{*}(t), \tilde{\pi}_{2}^{*}(t)\right) \\
\tilde{J}_{2}\left(t, x_{2}, x_{1}, \hat{\pi}_{2}(t), \tilde{\pi}_{1}^{*}(t)\right) \leq \tilde{J}_{2}\left(t, x_{2}, x_{1}, \tilde{\pi}_{2}^{*}(t), \tilde{\pi}_{1}^{*}(t)\right) .
\end{array}\right.
$$

The following theorem provides the Nash equilibrium for the case of the diffusion-approximated process $\left\{\hat{\mathcal{X}}_{k}^{\tilde{\pi}_{k}}(t)\right\}_{t \geq 0}$. 
Theorem 6.1. Assume that $\kappa_{1} \kappa_{2}<1$. Suppose that insurer $k$, for $k=1,2$, has an exponential utility function $U^{k}$ in (17) and the relative wealth process $\left\{\hat{\mathcal{X}}_{k}^{\tilde{\pi}_{k}}(t)\right\}_{t \geq 0}$ in (37). The value function $\tilde{V}^{k}(t, x, l)$ of insurer $k$, for $k=1,2$, at equilibrium is

$$
\tilde{V}^{k}(t, x, l)=-\frac{1}{q_{k}} \exp \left\{-q_{k}\left[x \mathrm{e}^{r(T-t)}+\tilde{f}^{k}(t)\right]-A(t) l-B(t)\right\},
$$

where $A(t), B(t)$ are given in (21), (22) and

$$
\begin{aligned}
\tilde{f}^{k}(t)= & {\left[\frac{\left(\lambda_{k}+\lambda\right)\left(\eta_{k}-\theta_{k}\right)}{r} \bar{\mu}_{k}-\frac{\kappa_{k}\left(\lambda_{j}+\lambda\right)\left(\eta_{j}-\theta_{j}\right)}{r} \bar{\mu}_{j}\right]\left[1-\mathrm{e}^{r(T-t)}\right] } \\
& -\left(\lambda_{k}+\lambda\right) \theta_{k} \int_{t}^{T} \mathrm{e}^{r(T-s)} \mu_{k}\left(\tilde{a}_{k}^{*}(s)\right) \mathrm{d} s+\kappa_{k}\left(\lambda_{j}+\lambda\right) \theta_{j} \int_{t}^{T} \mathrm{e}^{r(T-s)} \mu_{j}\left(\tilde{a}_{j}^{*}(s)\right) \mathrm{d} s \\
& +\left(\lambda_{k}+\lambda\right) q_{k} \int_{t}^{T} \mathrm{e}^{2 r(T-s)} \int_{0}^{\tilde{a}_{k}^{*}(s)} y \bar{F}_{k}(y) \mathrm{d} y \mathrm{~d} s \\
& +\kappa_{k}^{2}\left(\lambda_{j}+\lambda\right) q_{k} \int_{t}^{T} \mathrm{e}^{2 r(T-s)} \int_{0}^{\tilde{a}_{j}^{*}(s)} y \bar{F}_{j}(y) \mathrm{d} y \mathrm{~d} s \\
& -\lambda \kappa_{k} q_{k} \int_{t}^{T} \mathrm{e}^{2 r(T-s)}\left(\int_{0}^{\tilde{a}_{k}^{*}(s)} \bar{F}_{k}(y) \mathrm{d} y\right)\left(\int_{0}^{\tilde{a}_{j}^{*}(s)} \bar{F}_{j}(y) \mathrm{d} y\right) \mathrm{d} s,
\end{aligned}
$$

for $k, j=1,2$ with $k \neq j$. Let $\hat{\tilde{a}}_{k}$, for $k=1,2$, be the solutions to the following system of equations,

$$
\left\{\begin{array}{l}
\hat{\tilde{a}}_{1}(t)=\frac{\lambda \kappa_{1}}{\lambda_{1}+\lambda} \int_{0}^{\hat{a}_{2}(t)} \bar{F}_{2}(y) \mathrm{d} y+\frac{\theta_{1}}{q_{1}} \mathrm{e}^{-r(T-t)}, \\
\hat{\tilde{a}}_{2}(t)=\frac{\lambda \kappa_{2}}{\lambda_{2}+\lambda} \int_{0}^{\hat{\tilde{a}}_{1}(t)} \bar{F}_{1}(y) \mathrm{d} y+\frac{\theta_{2}}{q_{2}} \mathrm{e}^{-r(T-t)},
\end{array}\right.
$$

then the equilibrium reinsurance strategies $\hat{\tilde{a}}_{k}^{*}$, for $k=1,2$, for Problem 3.1 admit one of the following forms: for $k \neq j \in\{1,2\}$,

1. If $\hat{\tilde{a}}_{k}(t) \leq D_{k}$ and $\hat{\tilde{a}}_{j}(t) \leq D_{j}$, then $\left(\tilde{a}_{k}^{*}(t), \tilde{a}_{j}^{*}(t)\right)=\left(\hat{\tilde{a}}_{k}(t), \hat{\tilde{a}}_{j}(t)\right)$;

2. If $\hat{\tilde{a}}_{k}(t)>D_{k}$ and $\hat{\tilde{a}}_{j}(t) \leq D_{j}$, then $\left(\tilde{a}_{k}^{*}(t), \tilde{a}_{j}^{*}(t)\right)=\left(D_{k},\left[\frac{\lambda \kappa_{j}}{\lambda_{j}+\lambda} \int_{0}^{D_{k}} \bar{F}_{k}(y) \mathrm{d} y+\frac{\theta_{j}}{q_{j}} \mathrm{e}^{-r(T-t)}\right] \wedge D_{j}\right)$;

3. If $\hat{\tilde{a}}_{k}(t)>D_{k}$ and $\hat{\tilde{a}}_{j}(t)>D_{j}$, then $\left(\tilde{a}_{k}^{*}(t), \tilde{a}_{j}^{*}(t)\right)=\left(D_{k}, D_{j}\right)$;

and the investment strategies $\tilde{b}_{k}^{*}$, for $k=1,2$, are given by (24).

Proof. Following the notations and assumptions in Theorem 4.1 , let $\tilde{W}^{k}(t, x, l) \in \mathcal{C}^{1,2}(\Gamma) \cap$ $\mathcal{C}^{0}(\bar{\Gamma})$, for $k=1,2$, be a solution of the following HJB equation,

$$
\begin{aligned}
0= & \tilde{W}_{t}^{k}(t, x, l)+\sup _{\pi_{k} \in \Pi_{k}}\left\{\left[r x+\left(\lambda_{k}+\lambda\right)\left[\theta_{k} \mu_{k}\left(\tilde{a}_{k}\right)+\left(\eta_{k}-\theta_{k}\right) \bar{\mu}_{k}\right]\right.\right. \\
& \left.-\kappa_{k}\left(\lambda_{j}+\lambda\right)\left[\theta_{j} \mu_{j}\left(\hat{\tilde{a}}_{j}\right)+\left(\eta_{j}-\theta_{j}\right) \bar{\mu}_{j}\right]+m\left[\tilde{b}_{k}-\kappa_{k} \hat{\tilde{b}}_{j}\right] l\right] \tilde{W}_{x}^{k}(t, x, l) \\
& +\frac{1}{2}\left[\left(\lambda_{k}+\lambda\right)\left(\int_{0}^{\tilde{a}_{k}} 2 y \bar{F}_{k}(y) \mathrm{d} y\right)+\kappa_{k}^{2}\left(\lambda_{j}+\lambda\right)\left(\int_{0}^{\hat{\tilde{a}}_{j}} 2 y \bar{F}_{j}(y) \mathrm{d} y\right)\right.
\end{aligned}
$$




$$
\begin{aligned}
& \left.-2 \kappa_{k} \lambda\left(\int_{0}^{\tilde{a}_{k}} \bar{F}_{k}(y) \mathrm{d} y\right)\left(\int_{0}^{\hat{\tilde{a}}_{j}} \bar{F}_{j}(y) \mathrm{d} y\right)+\tilde{b}_{k}^{2} l+\kappa_{k}^{2} \hat{\tilde{b}}_{j}^{2} l-2 \kappa_{k} \tilde{b}_{k} \hat{\tilde{b}}_{j} l\right] \tilde{W}_{x x}^{k}(t, x, l) \\
& \left.+\alpha(\delta-l) \tilde{W}_{l}^{k}(t, x, l)+\frac{1}{2} \sigma^{2} l \tilde{W}_{l l}^{k}(t, x, l)+\rho_{S} \sigma l\left[\tilde{b}_{k}-\kappa_{k} \hat{\tilde{b}}_{j}\right] \tilde{W}_{x l}^{k}(t, x, l)\right\} .
\end{aligned}
$$

Consider the following Ansatz $W^{k}(t, x, l)$,

$$
\tilde{W}^{k}(t, x, l)=-\frac{1}{q_{k}} \exp \left\{-q_{k}\left[x \mathrm{e}^{r(T-t)}+\tilde{f}^{k}(t)+g^{k}(t, l)\right]\right\} .
$$

We shall prove the verification theorem for $\tilde{V}^{k}=\tilde{W}^{k}$ in Appendix A. Since

$$
\begin{aligned}
& \tilde{W}_{t}^{k}=\left(r q_{k} x \mathrm{e}^{r(T-t)}-q_{k} \tilde{f}_{t}^{k}-q_{k} g_{t}^{k}\right) \tilde{W}^{k}, \quad \tilde{W}_{x}^{k}=-q_{k} \mathrm{e}^{r(T-t)} \tilde{W}^{k}, \quad \tilde{W}_{x x}^{k}=q_{k}^{2} \mathrm{e}^{2 r(T-t)} \tilde{W}^{k}, \\
& \tilde{W}_{l}^{k}=-q_{k} g_{l}^{k} \tilde{W}^{k}, \quad W_{l l}^{k}=q_{k}^{2}\left(g_{l}^{k}\right)^{2} \tilde{W}^{k}, \quad \tilde{W}_{x l}^{k}=q_{k}^{2} \mathrm{e}^{r(T-t)} g_{l}^{k} \tilde{W}^{k},
\end{aligned}
$$

the corresponding HJB equation in (44) becomes

$$
\begin{aligned}
& 0=\left(r q_{k} x \mathrm{e}^{r(T-t)}-q_{k} \tilde{f}_{t}^{k}-q_{k} g_{t}^{k}\right) \tilde{W}^{k} \\
& +\sup _{\pi_{k} \in \Pi_{k}}\left\{\left[r x+\left(\lambda_{k}+\lambda\right)\left[\theta_{k} \mu_{k}\left(\tilde{a}_{k}\right)+\left(\eta_{k}-\theta_{k}\right) \bar{\mu}_{k}\right]-\kappa_{k}\left(\lambda_{j}+\lambda\right)\left[\theta_{j} \mu_{j}\left(\hat{\tilde{a}}_{j}\right)+\left(\eta_{j}-\theta_{j}\right) \bar{\mu}_{j}\right]\right.\right. \\
& \left.+m\left[\tilde{b}_{k}-\kappa_{k} \hat{\tilde{b}}_{j}\right] l\right]\left(-q_{k} \mathrm{e}^{r(T-t)} \tilde{W}^{k}\right)+\frac{1}{2}\left[\left(\lambda_{k}+\lambda\right)\left(\int_{0}^{\tilde{a}_{k}} 2 y \bar{F}_{k}(y) \mathrm{d} y\right)\right. \\
& +\kappa_{k}^{2}\left(\lambda_{j}+\lambda\right)\left(\int_{0}^{\hat{\tilde{a}}_{j}} 2 y \bar{F}_{j}(y) \mathrm{d} y\right)-2 \kappa_{k} \lambda\left(\int_{0}^{\tilde{a}_{k}} \bar{F}_{k}(y) \mathrm{d} y\right)\left(\int_{0}^{\hat{\tilde{a}}_{j}} \bar{F}_{j}(y) \mathrm{d} y\right) \\
& \left.+\tilde{b}_{k}^{2} l+\kappa_{k}^{2} \tilde{\tilde{b}}_{j}^{2}-2 \kappa_{k} \tilde{b}_{k} \hat{\tilde{b}}_{j} l\right]\left(q_{k}^{2} \mathrm{e}^{2 r(T-t)} \tilde{W}^{k}\right) \\
& \left.+\alpha(\delta-l)\left(-q_{k} g_{l}^{k} \tilde{W}^{k}\right)+\frac{1}{2} \sigma^{2} l\left(q_{k}^{2}\left(g_{l}^{k}\right)^{2} \tilde{W}^{k}\right)+\rho_{S} \sigma l\left[\tilde{b}_{k}-\kappa_{k} \hat{\tilde{b}}_{j}\right]\left(q_{k}^{2} \mathrm{e}^{r(T-t)} g_{l}^{k} \tilde{W}^{k}\right)\right\} \\
& =\left(r q_{k} x \mathrm{e}^{r(T-t)}-q_{k} \tilde{f}_{t}^{k}-q_{k} g_{t}^{k}\right)-\left(r x+\left(\lambda_{k}+\lambda\right)\left(\eta_{k}-\theta_{k}\right) \bar{\mu}_{k}\right. \\
& -\kappa_{k}\left(\lambda_{j}+\lambda\right)\left[\theta_{j} \mu_{j}\left(\hat{\tilde{a}}_{j}\right)+\left(\eta_{j}-\theta_{j}\right) \bar{\mu}_{j}\right] \\
& \left.-m \kappa_{k} \hat{\tilde{b}}_{j} l\right) q_{k} \mathrm{e}^{r(T-t)}+\frac{1}{2}\left[\kappa_{k}^{2}\left(\lambda_{j}+\lambda\right)\left(\int_{0}^{\hat{\tilde{a}}_{j}} 2 z \bar{F}_{j}(y) \mathrm{d} y\right)+\kappa_{k}^{2} \hat{\tilde{b}}_{j}^{2}\right]\left(q_{k}^{2} \mathrm{e}^{2 r(T-t)}\right) \\
& -\alpha(\delta-l) q_{k} g_{l}^{k}+\frac{1}{2} l \sigma^{2} q_{k}^{2}\left(g_{l}^{k}\right)^{2}-\rho_{S} \sigma l \kappa_{k} \hat{\tilde{b}}_{j} q_{k}^{2} g_{l}^{k} \mathrm{e}^{r(T-t)}+\sup _{\tilde{a}_{k}}\left\{-\left(\lambda_{k}+\lambda\right) \theta_{k} \mu_{k}\left(\tilde{a}_{k}\right) q_{k} \mathrm{e}^{r(T-t)}\right. \\
& \left.+\frac{1}{2}\left[\left(\lambda_{k}+\lambda\right)\left(\int_{0}^{\tilde{a}_{k}} 2 z \bar{F}_{k}(y) \mathrm{d} y\right)-2 \kappa_{k} \lambda\left(\int_{0}^{\tilde{a}_{k}} \bar{F}_{k}(y) \mathrm{d} y\right)\left(\int_{0}^{\hat{\tilde{a}}_{j}} \bar{F}_{j}(y) \mathrm{d} y\right)\right] q_{k}^{2} \mathrm{e}^{2 r(T-t)}\right\} \\
& +\sup _{\tilde{b}_{k}}\left\{-m \tilde{b}_{k} l q_{k} \mathrm{e}^{r(T-t)}+\frac{1}{2}\left[\tilde{b}_{k}^{2} l-2 \kappa_{k} \tilde{b}_{k} \hat{\tilde{b}}_{j} l\right] q_{k}^{2} \mathrm{e}^{2 r(T-t)}+\rho_{S} \sigma l \tilde{b}_{k} q_{k}^{2} g_{l}^{k} \mathrm{e}^{r(T-t)}\right\} .
\end{aligned}
$$

As it is easy to see that the investment strategy of insurer $k$ at equilibrium $\tilde{b}_{k}^{*}$, for $k=1,2$, admits the form in $(24)$, i.e $\tilde{b}_{k}^{*}=b_{k}^{*, \mathfrak{n}}$, where $b_{k}^{*, \mathfrak{n}}$ is given in Theorem 5.1 , we shall hereafter 
focus exclusively on the reinsurance strategies, $\tilde{a}_{k}^{*}$, for $k=1,2$. By the first order condition for $\tilde{a}_{k}$, we have

$$
0=-\left(\lambda_{k}+\lambda\right) \theta_{k} \bar{F}_{k}\left(\tilde{a}_{k}\right)+\left[\left(\lambda_{k}+\lambda\right) \tilde{a}_{k} \bar{F}_{k}\left(\tilde{a}_{k}\right)-\kappa_{k} \lambda \bar{F}_{k}\left(\tilde{a}_{k}\right)\left(\int_{0}^{\hat{\tilde{a}}_{j}} \bar{F}_{j}(y) \mathrm{d} y\right)\right] q_{k} \mathrm{e}^{r(T-t)}
$$

which gives

$$
\hat{\tilde{a}}_{k}=\frac{\kappa_{k} \lambda}{\lambda_{k}+\lambda}\left(\int_{0}^{\hat{\tilde{a}}_{j}} \bar{F}_{j}(y) \mathrm{d} y\right)+\frac{\theta_{k} \mathrm{e}^{-r(T-t)}}{q_{k}} .
$$

Hence, it follows that the desired equilibrium reinsurance strategy $\left(\tilde{a}_{1}^{*}, \tilde{a}_{2}^{*}\right)$ can be obtained when we can show that of the system of equations in (43) admits unique solution $\left(\hat{\tilde{a}}_{1}, \hat{\tilde{a}}_{2}\right)$. To solve for $\left(\hat{\tilde{a}}_{1}, \hat{\tilde{a}}_{2}\right)$, consider the following sequence $\left\{\hat{\tilde{a}}_{1, l}\right\}_{l=0}^{\infty}$ :

$$
\left\{\begin{array}{l}
\hat{\tilde{a}}_{1,0}(t) \triangleq \frac{\theta_{1} \mathrm{e}^{-r(T-t)}}{q_{1}}, \\
\hat{\tilde{a}}_{1, l}(t) \triangleq \frac{\kappa_{1} \lambda}{\lambda_{1}+\lambda} \int_{0}^{\hat{\tilde{a}}_{2, l-1}(t)} \bar{F}_{2}(y) \mathrm{d} y+\frac{\theta_{1} \mathrm{e}^{-r(T-t)}}{q_{1}}, \quad \text { for } \quad l \geq 1,
\end{array}\right.
$$

where

$$
\hat{\tilde{a}}_{2, l}(t)=\frac{\kappa_{2} \lambda}{\lambda_{2}+\lambda} \int_{0}^{\hat{\tilde{a}}_{1, l}(t)} \bar{F}_{1}(y) \mathrm{d} y+\frac{\theta_{2} \mathrm{e}^{-r(T-t)}}{q_{2}},
$$

for $l \in \mathbb{N}$.

Next, we show that $\left\{\hat{\tilde{a}}_{1, l}\right\}_{l=0}^{\infty}$ is a Cauchy sequence with respect to the uniform norm; indeed, note that

$$
\begin{aligned}
\left|\hat{\tilde{a}}_{1, l+1}(t)-\hat{\tilde{a}}_{1, l}(t)\right| & =\left|\frac{\kappa_{1} \lambda}{\lambda_{1}+\lambda} \int_{\hat{\tilde{a}}_{2, l-1}(t)}^{\hat{\tilde{a}}_{2, l}(t)} \bar{F}_{2}(y) \mathrm{d} y\right| \\
& \leq\left|\frac{\kappa_{1} \lambda}{\lambda_{1}+\lambda} \int_{\hat{\tilde{a}}_{2, l-1}(t)}^{\hat{\tilde{a}}_{2, l}(t)} \mathrm{d} y\right|=\frac{\kappa_{1} \lambda}{\lambda_{1}+\lambda}\left|\hat{\tilde{a}}_{2, l}(t)-\hat{\tilde{a}}_{2, l-1}(t)\right| .
\end{aligned}
$$

From (47), it is easy to see that $\hat{\tilde{a}}_{k, l}(t)$ is uniformly bounded for $k=1,2$, and $l \in \mathbb{N}$, and henceforth we have

$$
\left\|\hat{\tilde{a}}_{1, l+1}-\hat{\tilde{a}}_{1, l}\right\|_{\infty} \leq \frac{\kappa_{1} \lambda}{\lambda_{1}+\lambda}\left\|\hat{\tilde{a}}_{2, l}-\hat{\tilde{a}}_{2, l-1}\right\|_{\infty}
$$

By symmetry, we also have,

$$
\left\|\hat{\tilde{a}}_{2, l}-\hat{\tilde{a}}_{2, l-1}\right\|_{\infty} \leq \frac{\kappa_{2} \lambda}{\lambda_{2}+\lambda}\left\|\hat{\tilde{a}}_{1, l}-\hat{\tilde{a}}_{1, l-1}\right\|_{\infty} .
$$

This readily implies that

$$
\begin{aligned}
\left\|\hat{\tilde{a}}_{1, l+1}(t)-\hat{\tilde{a}}_{1, l}\right\|_{\infty} & \leq\left(\frac{\kappa_{1} \lambda}{\lambda_{1}+\lambda}\right)\left[\left(\frac{\kappa_{2} \lambda}{\lambda_{2}+\lambda}\right)\left\|\hat{\tilde{a}}_{1, l}-\hat{\tilde{a}}_{1, l-1}\right\|_{\infty}\right] \\
& \leq\left[\kappa_{1} \kappa_{2}\left(\frac{\lambda}{\lambda_{1}+\lambda}\right)\left(\frac{\lambda}{\lambda_{2}+\lambda}\right)\right]^{l}\left\|\hat{\tilde{a}}_{1,1}-\hat{\tilde{a}}_{1,0}\right\|_{\infty} \\
& \leq \tilde{\Upsilon}^{l}\left\|\hat{\tilde{a}}_{1,1}-\hat{\tilde{a}}_{1,0}\right\|_{\infty} .
\end{aligned}
$$


This shows that, given a large integer $n$, for any $m>n$, we have

$$
\begin{aligned}
\left\|\hat{\tilde{a}}_{1, m}-\hat{\tilde{a}}_{1, n}\right\|_{\infty} & \leq \sum_{l=n}^{\infty}\left\|\hat{\tilde{a}}_{1, l+1}(t)-\hat{\tilde{a}}_{1, l}(t)\right\|_{\infty} \\
& \leq \frac{\tilde{\Upsilon}^{n}}{1-\bar{\Upsilon}}\left\|\hat{\tilde{a}}_{1,1}(t)-\hat{\tilde{a}}_{1,0}(t)\right\|_{\infty},
\end{aligned}
$$

where

$$
\tilde{\Upsilon} \triangleq \kappa_{1} \kappa_{2}\left(\frac{\lambda}{\lambda_{1}+\lambda}\right)\left(\frac{\lambda}{\lambda_{2}+\lambda}\right)
$$

Based on the uniform boundedness of sequence $\left\{\hat{\tilde{a}}_{1, l}\right\}$ and $\tilde{\Upsilon}<1$ under the assumption $\kappa_{1} \kappa_{2}<1$, we can henceforth conclude that $\left\{\hat{\tilde{a}}_{1, l}\right\}$ is Cauchy sequence with respect to uniform norm. This means that the left and right-hand sides of system (47) converge uniformly to a limit $\hat{\tilde{a}}_{k}$, for $k=1,2$, when $l$ approaches to $\infty$. To show uniqueness of the limit $\hat{\tilde{a}}_{k}$, for $k=1,2$, assume that there exist more than one limits, i.e. $\tilde{a}_{k}$ and $\hat{\tilde{a}}_{k}$. We can then follow the similar arguments above leading to (48) to arrive with the desired contradiction.

Finally, substituting (46) and the expressions of $\tilde{a}_{k}^{*}$ and $\tilde{b}_{k}^{*}$ into (44) gives

$$
\begin{aligned}
0= & \tilde{f}_{t}^{k}+\left(\left(\lambda_{k}+\lambda\right)\left[\theta_{k} \mu_{k}\left(\tilde{a}_{k}^{*}\right)+\left(\eta_{k}-\theta_{k}\right) \bar{\mu}_{k}\right]-\kappa_{k}\left(\lambda_{j}+\lambda\right)\left[\theta_{j} \mu_{j}\left(\tilde{a}_{j}^{*}\right)+\left(\eta_{j}-\theta_{j}\right) \bar{\mu}_{j}\right]\right) \mathrm{e}^{r(T-t)} \\
& -\frac{1}{2}\left[\left(\lambda_{k}+\lambda\right)\left(\int_{0}^{\tilde{a}_{k}^{*}} 2 z \bar{F}_{k}(y) \mathrm{d} y\right)-2 \kappa_{k} \lambda\left(\int_{0}^{\tilde{a}_{k}^{*}} \bar{F}_{k}(y) \mathrm{d} y\right)\right. \\
& \left.+\left(\int_{0}^{\tilde{a}_{j}^{*}} \bar{F}_{j}(y) \mathrm{d} y\right) \kappa_{k}^{2}\left(\lambda_{j}+\lambda\right)\left(\int_{0}^{\tilde{a}_{j}^{*}} 2 z \bar{F}_{j}(y) \mathrm{d} y\right)\right] q_{k} \mathrm{e}^{2 r(T-t)},
\end{aligned}
$$

of which (42) follows readily.

Different from Theorem 5.1, Theorem 6.1 does not require additional assumption as to guarantee the reinsurance strategies, $\tilde{a}_{k}^{*}$, for $k=1,2$, to uniquely exist. This indicates the high analytical tractability of the diffusion-approximated process $\left\{\hat{\mathcal{X}}_{k}^{\tilde{\pi}_{k}}\right\}_{t>0}$ is maintained in the context of the nonzero-sum game framework. We now establish the connection between Nash equilibria under the general compound Poisson process $\left\{\hat{X}_{k}^{\pi_{k}^{\mathfrak{n}}}\right\}_{t>0}$ and and the associated diffusion-approximated process as $\mathfrak{n} \rightarrow \infty$. More specifically, the following theorem shows that the Nash equilibrium under the diffusion-approximated process is the $\epsilon$-Nash equilibrium for the general compound Poisson process.

Theorem 6.2. Assume that $C(\mathfrak{n})=O\left(\mathfrak{n}^{-\frac{\mathfrak{a}}{2}}\right)$. Under the condition that

$$
\limsup _{\mathfrak{n} \rightarrow \infty}\left(\max \left\{\left\|a_{1}^{*, \mathfrak{n}}\right\|_{\infty},\left\|a_{2}^{*, \mathfrak{n}}\right\|_{\infty}\right\}\right)<\infty
$$

then

1. For $k=1,2$,

$$
\left\|a_{k}^{*, \mathfrak{n}}-\tilde{a}_{k}^{*}\right\|_{\infty}=O\left(\mathfrak{n}^{-\frac{\mathfrak{a}}{2}}\right)
$$

where $a_{k}^{*, \mathfrak{n}}$ and $\tilde{a}_{k}^{*}$ are the equilibrium reinsurance strategies of insurer $k$ in Theorem 5.1 and Theorem 6.1, respectively; 
2. For each $\hat{x}_{k}, l \in \mathbb{R}$,

$$
\left\|V^{k, \mathfrak{n}}(., \hat{x}, l)-\tilde{V}^{k}(., \hat{x}, l)\right\|_{\infty}=O\left(\mathfrak{n}^{-\frac{\mathfrak{a}}{2}}\right),
$$

where the value functions $V^{k, \mathfrak{n}}$ and $\tilde{V}^{k}$ are the value functions of insurer $k$, for $k=1,2$, in (19) and (41), respectively.;

3. For each $x_{k}, x_{j} \in \mathbb{R}$, for $k=1,2$ and $k \neq j \in\{1,2\}$,

$$
\left\|J_{k}^{\mathfrak{n}}\left(., x_{k}, x_{j}, \pi_{k}^{*, \mathfrak{n}}, \pi_{j}^{*, \mathfrak{n}}\right)-J_{k}^{\mathfrak{n}}\left(., x_{k}, x_{j}, \tilde{\pi}_{k}, \tilde{\pi}_{j}\right)\right\|_{\infty}=O\left(\mathfrak{n}^{-\frac{\mathfrak{a}}{2}}\right),
$$

i.e. Nash equilibrium under the diffusion-approximated process $\left(\tilde{\pi}_{1}, \tilde{\pi}_{2}\right)$ serves as an $\epsilon$-Nash equilibrium under the general Poisson process $\left(\pi_{1}^{*, \mathfrak{n}}, \pi_{2}^{*, \mathfrak{n}}\right)$.

Proof. Without any loss of generality, we let $\mathcal{K}<\infty$ to be some constant such that

$$
\limsup _{\mathfrak{n} \rightarrow \infty}\left(\max \left\{\left\|a_{1}^{*, \mathfrak{n}}\right\|_{\infty},\left\|a_{2}^{*, \mathfrak{n}}\right\|_{\infty}\right\}\right)<\mathcal{K} .
$$

From (53), it follows that there exists an $\mathfrak{n}^{*} \in \mathbb{N}$, such that for all $\mathfrak{n}>\mathfrak{n}^{*}$,

$$
\begin{aligned}
\left|\mathfrak{n}^{\mathfrak{a}} C(\mathfrak{n}) h_{k}^{\mathfrak{n}}\left(a_{j}^{*, \mathfrak{n}}(t)\right)\right| & =\mathfrak{n}^{\mathfrak{a}} C(\mathfrak{n})\left|\int_{0}^{a_{j}^{*, \mathfrak{n}}(t)} \kappa_{k} q_{k} \mathrm{e}^{r(T-t)} C(\mathfrak{n}) \mathrm{e}^{-\kappa_{k} q_{k} \mathrm{e}^{r(T-t)} C(\mathfrak{n}) y} \bar{F}_{j}(y) \mathrm{d} y\right| \\
& \leq \mathfrak{n}^{\mathfrak{a}} C(\mathfrak{n})\left|\int_{0}^{a_{j}^{*, \mathfrak{n}}(t)} \kappa_{k} q_{k} \mathrm{e}^{r(T-t)} C(\mathfrak{n}) \mathrm{e}^{-\kappa_{k} q_{k} \mathrm{e}^{r(T-t)} C(\mathfrak{n}) y} \mathrm{~d} y\right| \\
& =\mathfrak{n}^{\mathfrak{a}} C(\mathfrak{n}) \kappa_{k} q_{k} \mathrm{e}^{r(T-t)} C(\mathfrak{n}) \mathrm{e}^{-\kappa_{k} q_{k} \mathrm{e}^{r(T-t)} C(\mathfrak{n}) a_{0}}\left|a_{j}^{*, \mathfrak{n}}(t)\right| \\
& \leq \mathfrak{n}^{\mathfrak{a}}(C(\mathfrak{n}))^{2} \kappa_{k} q_{k} \mathrm{e}^{r T} \mathcal{K}=O(1),
\end{aligned}
$$

where the second equality follows from the mean-value theorem for some $a_{0} \in\left[0, a_{j}^{*, \mathfrak{n}}(t)\right]$. Since $-1 \leq h_{k}^{\mathfrak{n}}(a) \leq 0$, for all $a \geq 0$, together with (54), it follows readily that

$$
\left[\frac{\left(\lambda_{k}+\lambda\right) \theta_{k}-\lambda \mathfrak{n}^{\mathfrak{a}} C(\mathfrak{n}) h_{k}^{\mathfrak{n}}\left(a_{j}^{*, \mathfrak{n}}(t)\right)}{\lambda_{k}+\lambda\left[h_{k}^{\mathfrak{n}}\left(a_{j}^{*, \mathfrak{n}}(t)\right)+1\right]}\right]=O(1),
$$

for all $\mathfrak{n}>\mathfrak{n}^{*}$. Therefore, for all $\mathfrak{n}>\mathfrak{n}^{*}, a_{k}^{*, \mathfrak{n}}(t)$ can be rewritten as follows, by first expanding it as a Taylor series,

$$
\begin{aligned}
a_{k}^{*, \mathfrak{n}}(t)= & \frac{\mathrm{e}^{-r(T-t)}}{q_{k} C(\mathfrak{n})} \ln \left\{\frac{\left(\lambda_{k}+\lambda\right)\left(1+\frac{\theta_{k}}{\mathfrak{n}^{\mathfrak{a}} C(\mathfrak{n})}\right)}{\lambda_{k}+\lambda\left[h_{k}^{\mathfrak{n}}\left(a_{j}^{*, \mathfrak{n}}(t)\right)+1\right]}\right\} \\
= & \frac{\mathrm{e}^{-r(T-t)}}{q_{k} C(\mathfrak{n})}\left\{\left(\frac{1}{\mathfrak{n}^{\mathfrak{a}} C(\mathfrak{n})}\right)\left[\frac{\left(\lambda_{k}+\lambda\right) \theta_{k}-\lambda \mathfrak{n}^{\mathfrak{a}} C(\mathfrak{n}) h_{k}^{\mathfrak{n}}\left(a_{j}^{*, \mathfrak{n}}(t)\right)}{\lambda_{k}+\lambda\left[h_{k}^{\mathfrak{n}}\left(a_{j}^{*, \mathfrak{n}}(t)\right)+1\right]}\right]\right. \\
& \left.-\left(\frac{1}{2 \mathfrak{n}^{2 \mathfrak{a}}(C(\mathfrak{n}))^{2}}\right)\left[\frac{\left(\lambda_{k}+\lambda\right) \theta_{k}-\lambda \mathfrak{n}^{\mathfrak{a}} C(\mathfrak{n}) h_{k}^{\mathfrak{n}}\left(a_{j}^{*, \mathfrak{n}}(t)\right)}{\lambda_{k}+\lambda\left[h_{k}^{\mathfrak{n}}\left(a_{j}^{*, \mathfrak{n}}(t)\right)+1\right]}\right]^{2}+O\left(\mathfrak{n}^{-\frac{3 \mathfrak{a}}{2}}\right)\right\} \\
= & \frac{\mathrm{e}^{-r(T-t)}}{q_{k} \mathfrak{n}^{\mathfrak{a}}(C(\mathfrak{n}))^{2}}\left[\frac{\left(\lambda_{k}+\lambda\right) \theta_{k}-\lambda \mathfrak{n}^{\mathfrak{a}} C(\mathfrak{n}) h_{k}^{\mathfrak{n}}\left(a_{j}^{*, \mathfrak{n}}(t)\right)}{\lambda_{k}+\lambda\left[h_{k}^{\mathfrak{n}}\left(a_{j}^{*, \mathfrak{n}}(t)\right)+1\right]}\right]+O\left(\mathfrak{n}^{-\frac{\mathfrak{a}}{2}}\right)
\end{aligned}
$$


Let $\tilde{\mathcal{K}} \triangleq \max \left\{\left\|\tilde{a}_{1}^{*}\right\|_{\infty},\left\|\tilde{a}_{2}^{*}\right\|_{\infty}\right\}$. Combining (55) with $\tilde{a}_{1}^{*}(t)$ in (43), we have, for all $\mathfrak{n}>\mathfrak{n}^{*}$,

$$
\begin{aligned}
& \left|a_{1}^{*, \mathfrak{n}}(t)-\tilde{a}_{1}^{*}(t)\right| \leq \mid \frac{\mathrm{e}^{-r(T-t)}}{q_{1}(C(\mathfrak{n}))^{2} \mathfrak{n}^{\mathfrak{a}}}\left[\frac{\left(\lambda_{1}+\lambda\right) \theta_{1}}{\lambda_{1}+\lambda\left[h_{1}^{\mathfrak{n}}\left(a_{2}^{*, \mathfrak{n}}(t)\right)+1\right]}\right]-\frac{\theta_{1}}{q_{1}} \mathrm{e}^{-r(T-t)} \\
& -\frac{\mathrm{e}^{-r(T-t)}}{q_{1} C(\mathfrak{n})} \frac{\lambda h_{1}^{\mathfrak{n}}\left(a_{2}^{*, \mathfrak{n}}(t)\right)}{\lambda_{1}+\lambda\left[h_{1}^{\mathfrak{n}}\left(a_{2}^{*, \mathfrak{n}}(t)\right)+1\right]}-\frac{\lambda \kappa_{1}}{\lambda_{1}+\lambda} \int_{0}^{\tilde{a}_{2}(t)} \bar{F}_{2}(y) \mathrm{d} y \mid+O\left(\mathfrak{n}^{-\frac{\mathfrak{a}}{2}}\right) \\
& \leq \frac{\mathrm{e}^{-r(T-t)} \theta_{1}}{q_{1}(C(\mathfrak{n}))^{2} \mathfrak{n}^{\mathfrak{a}}} \frac{\left(\lambda_{1}+\lambda\right)\left|1-(C(\mathfrak{n}))^{2} \mathfrak{n}^{\mathfrak{a}}\right|}{\left(\lambda_{1}+\lambda\left[h_{1}^{\mathfrak{n}}\left(a_{2}^{*, \mathfrak{n}}(t)\right)+1\right]\right)} \\
& +\frac{\mathrm{e}^{-r(T-t)} \lambda \theta_{1}}{q_{1}} \frac{\left|\int_{0}^{a_{2}^{*, \mathfrak{n}}(t)} \kappa_{1} q_{1} \mathrm{e}^{r(T-t)} C(\mathfrak{n}) \mathrm{e}^{-\kappa_{1} q_{1} \mathrm{e}^{r(T-t)} C(\mathfrak{n}) y} \bar{F}_{2}(y) \mathrm{d} y\right|}{\left(\lambda_{1}+\lambda\left[h_{1}^{\mathfrak{n}}\left(a_{2}^{*, \mathfrak{n}}(t)\right)+1\right]\right)} \\
& +\frac{\mathrm{e}^{-r(T-t)}}{q_{1} C(\mathfrak{n})}\left[\frac{\lambda}{\lambda_{1}+\lambda\left[h_{1}^{\mathfrak{n}}\left(a_{2}^{*, \mathfrak{n}}(t)\right)+1\right]}\right] \\
& \cdot\left|\int_{\tilde{a}_{2}(t)}^{a_{2}^{*, \mathfrak{n}}(t)} \kappa_{1} q_{1} \mathrm{e}^{r(T-t)} C(\mathfrak{n}) \mathrm{e}^{-\kappa_{1} q_{1} \mathrm{e}^{r(T-t)} C(\mathfrak{n}) y} \bar{F}_{2}(y) \mathrm{d} y\right| \\
& +\left[\frac{\lambda \kappa_{1}}{\lambda_{1}+\lambda\left[h_{1}^{\mathfrak{n}}\left(a_{2}^{*, \mathfrak{n}}(t)\right)+1\right]}\right] \int_{0}^{\tilde{a}_{2}(t)}\left|\mathrm{e}^{-\kappa_{1} q_{1} \mathrm{e}^{r(T-t)} C(\mathfrak{n}) y}-1\right| \bar{F}_{2}(y) \mathrm{d} y \\
& +\left|\frac{\lambda \kappa_{1}}{\lambda_{1}+\lambda\left[h_{1}^{\mathfrak{n}}\left(a_{2}^{*, \mathfrak{n}}(t)\right)+1\right]}-\frac{\lambda \kappa_{1}}{\lambda_{1}+\lambda}\right| \int_{0}^{\tilde{a}_{2}(t)} \bar{F}_{2}(y) \mathrm{d} y+O\left(\mathfrak{n}^{-\frac{\mathfrak{a}}{2}}\right) \\
& \leq \frac{\mathrm{e}^{-r(T-t)} \theta_{1}}{q_{1}(C(\mathfrak{n}))^{2} \mathfrak{n}^{\mathfrak{a}}} \frac{\left(\lambda_{1}+\lambda\right)\left|1-(C(\mathfrak{n}))^{2} \mathfrak{n}^{\mathfrak{a}}\right|}{\left(\lambda_{1}+\lambda\left[h_{1}^{\mathfrak{n}}\left(a_{2}^{*, \mathfrak{n}}(t)\right)+1\right]\right)} \\
& +\frac{\mathrm{e}^{-r(T-t)} \lambda \theta_{1}}{q_{1}} \frac{\left|\int_{0}^{a_{2}^{*, \mathfrak{n}}(t)} \kappa_{1} q_{1} \mathrm{e}^{r(T-t)} C(\mathfrak{n}) \mathrm{e}^{-\kappa_{1} q_{1} \mathrm{e}^{r(T-t)} C(\mathfrak{n}) y} \mathrm{~d} y\right|}{\left(\lambda_{1}+\lambda\left[h_{1}^{\mathfrak{n}}\left(a_{2}^{*, \mathfrak{n}}(t)\right)+1\right]\right)} \\
& +\frac{\mathrm{e}^{-r(T-t)}}{q_{1} C(\mathfrak{n})}\left[\frac{\lambda}{\lambda_{1}+\lambda\left[h_{1}^{\mathfrak{n}}\left(a_{2}^{*, \mathfrak{n}}(t)\right)+1\right]}\right]\left|\int_{\tilde{a}_{2}(t)}^{a_{2}^{*, \mathfrak{n}}(t)} \kappa_{1} q_{1} \mathrm{e}^{r(T-t)} C(\mathfrak{n}) \mathrm{e}^{-\kappa_{1} q_{1} \mathrm{e}^{r(T-t)} C(\mathfrak{n}) y} \mathrm{~d} y\right| \\
& +\left[\frac{\lambda \kappa_{1}}{\lambda_{1}+\lambda\left[h_{1}^{\mathfrak{n}}\left(a_{2}^{*, \mathfrak{n}}(t)\right)+1\right]}\right] \int_{0}^{\tilde{a}_{2}(t)}\left|\mathrm{e}^{-\kappa_{1} q_{1} \mathrm{e}^{r(T-t)} C(\mathfrak{n}) y}-1\right| \mathrm{d} y \\
& +\frac{\lambda^{2} \kappa_{1}\left|\int_{0}^{a_{2}^{*, \mathfrak{n}}(t)} \kappa_{1} q_{1} \mathrm{e}^{r(T-t)} C(\mathfrak{n}) \mathrm{e}^{-\kappa_{1} q_{1} \mathrm{e}^{r(T-t)} C(\mathfrak{n}) y} \mathrm{~d} y\right|}{\left(\lambda_{1}+\lambda\left[h_{1}^{\mathfrak{n}}\left(a_{2}^{*, \mathfrak{n}}(t)\right)+1\right]\right)\left(\lambda_{1}+\lambda\right)} \tilde{a}_{2}(t)+O\left(\mathfrak{n}^{-\frac{\mathfrak{a}}{2}}\right) \\
& \leq \frac{\mathrm{e}^{-r(T-t)} \theta_{1}}{q_{1}(C(\mathfrak{n}))^{2} \mathfrak{n}^{\mathfrak{a}}} \frac{\left(\lambda_{1}+\lambda\right)\left|1-(C(\mathfrak{n}))^{2} \mathfrak{n}^{\mathfrak{a}}\right|}{\left(\lambda_{1}+\lambda\left[h_{1}^{\mathfrak{n}}\left(a_{2}^{*, \mathfrak{n}}(t)\right)+1\right]\right)}+\frac{\kappa_{1} \lambda \theta_{1} a_{2}^{*, \mathfrak{n}}(t)}{\left(\lambda_{1}+\lambda\left[h_{1}^{\mathfrak{n}}\left(a_{2}^{*, \mathfrak{n}}(t)\right)+1\right]\right)} C(\mathfrak{n}) \\
& +\frac{\lambda \kappa_{1}}{\lambda_{1}+\lambda\left[h_{1}^{\mathfrak{n}}\left(a_{2}^{*, \mathfrak{n}}(t)\right)+1\right]}\left|a_{2}^{*, \mathfrak{n}}(t)-\tilde{a}_{2}(t)\right|+\frac{2 \lambda \kappa_{1}^{2} q_{1} \mathrm{e}^{r(T-t)} C(\mathfrak{n})}{\lambda_{1}+\lambda\left[h_{1}^{\mathfrak{n}}\left(a_{2}^{*, \mathfrak{n}}(t)\right)+1\right]} \int_{0}^{\tilde{a}_{2}(t)} y \mathrm{~d} y \\
& +\frac{\lambda^{2} \kappa_{1}^{2} q_{1} \mathrm{e}^{r(T-t)} a_{2}^{*, \mathfrak{n}}(t) \tilde{a}_{2}(t)}{\left(\lambda_{1}+\lambda\left[h_{1}^{\mathfrak{n}}\left(a_{2}^{*, \mathfrak{n}}(t)\right)+1\right]\right)\left(\lambda_{1}+\lambda\right)} C(\mathfrak{n})+O\left(\mathfrak{n}^{-\frac{\mathfrak{a}}{2}}\right)
\end{aligned}
$$




$$
\begin{aligned}
\leq & \frac{\mathrm{e}^{-r(T-t)} \theta_{1}}{q_{1}(C(\mathfrak{n}))^{2} \mathfrak{n}^{\mathfrak{a}}} \frac{\left(\lambda_{1}+\lambda\right)\left|1-(C(\mathfrak{n}))^{2} \mathfrak{n}^{\mathfrak{a}}\right|}{\left(\lambda_{1}+\lambda\left[h_{1}^{\mathfrak{n}}\left(a_{2}^{*, \mathfrak{n}}(t)\right)+1\right]\right)}+\frac{\kappa_{1} \lambda \theta_{1} \mathcal{K}}{\left(\lambda_{1}+\lambda\left[h_{1}^{\mathfrak{n}}\left(a_{2}^{*, \mathfrak{n}}(t)\right)+1\right]\right)} C(\mathfrak{n}) \\
& +\frac{\lambda \kappa_{1}}{\lambda_{1}+\lambda\left[h_{1}^{\mathfrak{n}}\left(a_{2}^{*, \mathfrak{n}}(t)\right)+1\right]}\left|a_{2}^{*, \mathfrak{n}}(t)-\tilde{a}_{2}(t)\right|+\frac{\lambda \kappa_{1}^{2} q_{1} \mathrm{e}^{r(T-t)} \tilde{\mathcal{K}}^{2}}{\lambda_{1}+\lambda\left[h_{1}^{\mathfrak{n}}\left(a_{2}^{*, \mathfrak{n}}(t)\right)+1\right]} C(\mathfrak{n}) \\
& +\frac{\lambda^{2} \kappa_{1}^{2} q_{1} \mathrm{e}^{r(T-t)} \mathcal{K} \tilde{\mathcal{K}}}{\left(\lambda_{1}+\lambda\left[h_{1}^{\mathfrak{n}}\left(a_{2}^{*, \mathfrak{n}}(t)\right)+1\right]\right)\left(\lambda_{1}+\lambda\right)} C(\mathfrak{n})+O\left(\mathfrak{n}^{-\frac{\mathfrak{a}}{2}}\right) \\
= & \frac{\lambda \kappa_{1}}{\lambda_{1}+\lambda\left[h_{1}^{\mathfrak{n}}\left(a_{2}^{*, \mathfrak{n}}(t)\right)+1\right]}\left|a_{2}^{*, \mathfrak{n}}(t)-\tilde{a}_{2}(t)\right|+O\left(\mathfrak{n}^{-\frac{\mathfrak{a}}{2}}\right),
\end{aligned}
$$

where the third inequality follows from the fact that $0 \leq \bar{F}_{2}(y) \leq 1$, for $y \geq 0$; the fourth inequality follows from the mean-value theorem, i.e., for any $a, b \geq 0$, there exists $c \in$ $[a \wedge b, a \vee b]$ such that

$$
\left|h_{1}^{\mathfrak{n}}(a)-h_{1}^{\mathfrak{n}}(b)\right|=\kappa_{1} q_{1} \mathrm{e}^{r(T-t)} C(\mathfrak{n}) \mathrm{e}^{-\kappa_{1} q_{1} \mathrm{e}^{r(T-t)} C(\mathfrak{n}) c}|b-a| \leq \kappa_{1} q_{1} \mathrm{e}^{r(T-t)} C(\mathfrak{n})|b-a| ;
$$

the fifth inequality follows from the definitions of the upper bounds $\mathcal{K}$ and $\tilde{\mathcal{K}}$; and the sixth equality follows from the assumption that $C(\mathfrak{n})=O\left(\mathfrak{n}^{-\frac{\mathfrak{a}}{2}}\right)$ and the fact that $-1 \leq h_{1}^{\mathfrak{n}}(a) \leq 0$, for all $a \geq 0$.

By symmetry, the above inequality becomes

$$
\begin{aligned}
\left\|a_{1}^{*, \mathfrak{n}}-\tilde{a}_{1}^{*}\right\|_{\infty} & \leq\left\|\frac{\lambda \kappa_{1}}{\lambda_{1}+\lambda\left[h_{1}^{\mathfrak{n}}\left(a_{2}^{*, \mathfrak{n}}\right)+1\right]}\right\|_{\infty}\left\|a_{2}^{*, \mathfrak{n}}-\tilde{a}_{2}\right\|_{\infty}+O\left(\mathfrak{n}^{-\frac{\mathfrak{a}}{2}}\right) \\
& \leq\left\|\frac{\lambda^{2} \kappa_{1} \kappa_{2}}{\left(\lambda_{1}+\lambda\left[h_{1}^{\mathfrak{n}}\left(a_{2}^{*, \mathfrak{n}}\right)+1\right]\right)\left(\lambda_{2}+\lambda\left[h_{2}^{\mathfrak{n}}\left(a_{1}^{*, \mathfrak{n}}\right)+1\right]\right)}\right\|_{\infty}\left\|a_{1}^{*, \mathfrak{n}}-\tilde{a}_{1}\right\|_{\infty}+O\left(\mathfrak{n}^{-\frac{\mathfrak{a}}{2}}\right) \\
& =\mathcal{Y}_{\mathfrak{n}}\left\|a_{1}^{*, \mathfrak{n}}-\tilde{a}_{1}\right\|_{\infty}+O\left(\mathfrak{n}^{-\frac{\mathfrak{a}}{2}}\right)
\end{aligned}
$$

where $\mathcal{Y}_{\mathfrak{n}} \triangleq\left\|\frac{\lambda^{2} \kappa_{1} \kappa_{2}}{\left(\lambda_{1}+\lambda\left[h_{1}^{\mathfrak{n}}\left(a_{2}^{*, \mathfrak{n}}\right)+1\right]\right)\left(\lambda_{2}+\lambda\left[h_{2}^{\mathfrak{n}}\left(a_{1}^{*, \mathfrak{n}}\right)+1\right]\right)}\right\|_{\infty}$. From $(56)$, for $a>0$, there exists $\tilde{a} \in[0, a]$ such that, for $\mathfrak{n}>\mathfrak{n}^{*}$,

$$
\left.\mid h_{1}^{\mathfrak{n}}(a)\right)\left|=\kappa_{1} q_{1} \mathrm{e}^{r(T-t)} C(\mathfrak{n}) \mathrm{e}^{-\kappa_{1} q_{1} \mathrm{e}^{r(T-t)} C(\mathfrak{n}) \tilde{a}}\right| a \mid<\kappa_{1} q_{1} \mathrm{e}^{r T} C(\mathfrak{n}) \mathcal{K}=O(C(\mathfrak{n})),
$$

where the last inequality follows from condition (53). Therefore, for $\mathfrak{n}>\mathfrak{n}^{*}$,

$$
\begin{aligned}
& \left\|\frac{\lambda^{2} \kappa_{1} \kappa_{2}}{\left(\lambda_{1}+\lambda\left[h_{1}^{\mathfrak{n}}\left(a_{2}^{*, \mathfrak{n}}\right)+1\right]\right)\left(\lambda_{2}+\lambda\left[h_{2}^{\mathfrak{n}}\left(a_{1}^{*, \mathfrak{n}}\right)+1\right]\right)}-\frac{\lambda^{2} \kappa_{1} \kappa_{2}}{\left(\lambda_{1}+\lambda\right)\left(\lambda_{2}+\lambda\right)}\right\|_{\infty} \\
= & \left\|\frac{\lambda\left(\lambda_{1}+\lambda\right) h_{1}^{\mathfrak{n}}\left(a_{2}^{*, \mathfrak{n}}\right)+\lambda\left(\lambda_{2}+\lambda\right) h_{2}^{\mathfrak{n}}\left(a_{1}^{*, \mathfrak{n}}\right)-\lambda^{2} h_{1}^{\mathfrak{n}}\left(a_{2}^{*, \mathfrak{n}}\right) h_{2}^{\mathfrak{n}}\left(a_{1}^{*, \mathfrak{n}}\right)}{\left(\lambda_{1}+\lambda\right)\left(\lambda_{2}+\lambda\right)\left(\lambda_{1}+\lambda\left[h_{1}^{\mathfrak{n}}\left(a_{2}^{*, \mathfrak{n}}\right)+1\right]\right)\left(\lambda_{2}+\lambda\left[h_{2}^{\mathfrak{n}}\left(a_{1}^{*, \mathfrak{n}}\right)+1\right]\right)}\right\|_{\infty}=O(C(\mathfrak{n})),
\end{aligned}
$$

i.e. $\lim \sup _{\mathfrak{n} \rightarrow \infty} \mathcal{Y}_{\mathfrak{n}}=\frac{\lambda^{2} \kappa_{1} \kappa_{2}}{\left(\lambda_{1}+\lambda\right)\left(\lambda_{2}+\lambda\right)}<1$. Therefore, (57) becomes, for all $\mathfrak{n}>\mathfrak{n}^{*}$,

$$
\left\|a_{1}^{*, \mathfrak{n}}-\tilde{a}_{1}^{*}\right\|_{\infty} \leq \frac{1}{1-\mathcal{Y}_{\mathfrak{n}}} O\left(\mathfrak{n}^{-\frac{\mathfrak{a}}{2}}\right)=O\left(\mathfrak{n}^{-\frac{\mathfrak{a}}{2}}\right) .
$$


In view of $V^{k, \mathfrak{n}}$ in (19) and $\tilde{V}^{k}$ in (39), to show (51), it suffices to show that

$$
\left\|\tilde{f}^{k, \mathfrak{n}}-f^{k, \mathfrak{n}}\right\|_{\infty}=O\left(\mathfrak{n}^{-\frac{\mathfrak{a}}{2}}\right), \quad \text { for } \quad k=1,2 .
$$

For $\mathfrak{n}>\mathfrak{n}^{*},\left\|a_{k}^{*, \mathfrak{n}}\right\|_{\infty}<\mathcal{K}$, for $k=1,2$, and hence we have, for $0 \leq s \leq T$,

$$
\left\{\begin{array}{l}
\mathrm{e}^{q_{k} C(\mathfrak{n}) y \mathrm{e}^{r(T-s)}}=1+q_{k} C(\mathfrak{n}) y \mathrm{e}^{r(T-s)}+O\left(\mathfrak{n}^{-\mathfrak{a}}\right), \quad \text { for } \quad 0 \leq y \leq a_{k}^{* \mathfrak{n}}(s), \\
\mathrm{e}^{-\kappa_{k} q_{k} C(\mathfrak{n}) y \mathrm{e}^{r(T-s)}}=1-\kappa_{k} q_{k} C(\mathfrak{n}) y \mathrm{e}^{r(T-s)}+O\left(\mathfrak{n}^{-\mathfrak{a}}\right), \quad \text { for } \quad 0 \leq y \leq a_{j}^{*, \mathfrak{n}}(s) .
\end{array}\right.
$$

Substituting (59) into $f^{k, \mathfrak{n}}$ in (20), we then have:

$$
\begin{aligned}
f^{k, \mathfrak{n}}(t)= & {\left[\frac{\left(\lambda_{k}+\lambda\right)\left(\eta_{k}-\theta_{k}\right)}{r} \bar{\mu}_{k}-\frac{\kappa_{k}\left(\lambda_{j}+\lambda\right)\left(\eta_{j}-\theta_{j}\right)}{r} \bar{\mu}_{j}\right]\left[1-\mathrm{e}^{r(T-t)}\right] } \\
& -\left(\lambda_{k}+\lambda\right) \theta_{k} \int_{t}^{T} \mathrm{e}^{r(T-s)} \mu_{k}\left(a_{k}^{*, \mathfrak{n}}(s)\right) \mathrm{d} s+\kappa_{k}\left(\lambda_{j}+\lambda\right) \theta_{j} \int_{t}^{T} \mathrm{e}^{r(T-s)} \mu_{j}\left(a_{j}^{*, \mathfrak{n}}(s)\right) \mathrm{d} s \\
& +\left(\lambda_{k}+\lambda\right) \mathfrak{n}^{\mathfrak{a}}(C(\mathfrak{n}))^{2} q_{k} \int_{t}^{T} \mathrm{e}^{2 r(T-s)}\left[\int_{0}^{a_{k}^{*, \mathfrak{n}}(s)} y \bar{F}_{k}(y) \mathrm{d} y\right] \mathrm{d} s \\
& +\left(\lambda_{j}+\lambda\right) \mathfrak{n}^{\mathfrak{a}}(C(\mathfrak{n}))^{2} \kappa_{k}^{2} q_{k} \int_{t}^{T} \mathrm{e}^{2 r(T-s)}\left[\int_{0}^{a_{j}^{*, \mathfrak{n}}(s)} y \bar{F}_{j}(y) \mathrm{d} y\right] \mathrm{d} s \\
& -\lambda \mathfrak{n}^{\mathfrak{a}}(C(\mathfrak{n}))^{2} \kappa_{k} q_{k} \int_{t}^{T} \mathrm{e}^{2 r(T-s)}\left[\int_{0}^{a_{k}^{*, \mathfrak{n}}(s)} \bar{F}_{k}(y) \mathrm{d} y\right]\left[\int_{0}^{a_{j}^{*, \mathfrak{n}}(s)} \bar{F}_{j}(y) \mathrm{d} y\right] \mathrm{d} s \\
& +\lambda \mathfrak{n}^{\mathfrak{a}}(C(\mathfrak{n}))^{3} \kappa_{k}^{2} q_{k}^{2} \int_{t}^{T} \mathrm{e}^{3 r(T-s)}\left[\int_{0}^{a_{k}^{*, \mathfrak{n}}(s)} \bar{F}_{k}(y) \mathrm{d} y\right]\left[\int_{0}^{a_{j}^{*, \mathfrak{n}}(s)} y \bar{F}_{j}(y) \mathrm{d} y\right] \mathrm{d} s \\
& -\lambda \mathfrak{n}^{\mathfrak{a}}(C(\mathfrak{n}))^{3} \kappa_{k} q_{k}^{2} \int_{t}^{T} \mathrm{e}^{3 r(T-s)}\left[\int_{0}^{a_{k}^{*, \mathfrak{n}}(s)} y \bar{F}_{k}(y) \mathrm{d} y\right]\left[\int_{0}^{a_{j}^{*, \mathfrak{n}}(s)} \bar{F}_{j}(y) \mathrm{d} y\right] \mathrm{d} s \\
& +\lambda \mathfrak{n}^{\mathfrak{a}}(C(\mathfrak{n}))^{4} \kappa_{k}^{2} q_{k}^{3} \int_{t}^{T} \mathrm{e}^{4 r(T-s)}\left[\int_{0}^{a_{k}^{*, \mathfrak{n}}(s)} y \bar{F}_{k}(y) \mathrm{d} y\right]\left[\int_{0}^{a_{j}^{*, \mathfrak{n}}(s)} y \bar{F}_{j}(y) \mathrm{d} y\right] \mathrm{d} s \\
& +O\left(\mathfrak{n}^{-\frac{\mathfrak{a}}{2}}\right) .
\end{aligned}
$$

Combining (60) with $\tilde{f}^{k}$ in (42), direct calculation yields that, for $\mathfrak{n}>\mathfrak{n}^{*}$,

$$
\begin{aligned}
\left|\tilde{f}^{k}(t)-f^{k, \mathfrak{n}}(t)\right| \leq & \left(\lambda_{k}+\lambda\right) \theta_{k} \int_{t}^{T} \mathrm{e}^{r(T-s)}\left|\mu_{k}\left(\tilde{a}_{k}^{*}(s)\right)-\mu_{k}\left(a_{k}^{*, \mathfrak{n}}(s)\right)\right| \mathrm{d} s \\
& +\kappa_{k}\left(\lambda_{j}+\lambda\right) \theta_{j} \int_{t}^{T} \mathrm{e}^{r(T-s)}\left|\mu_{j}\left(\tilde{a}_{j}^{*}(s)\right)-\mu_{j}\left(a_{j}^{*, \mathfrak{n}}(s)\right)\right| \mathrm{d} s \\
& +\left(\lambda_{k}+\lambda\right) q_{k} \int_{t}^{T} \mathrm{e}^{2 r(T-s)}\left|\int_{a_{k}^{*, \mathfrak{n}}(s)}^{\tilde{a}_{k}^{*}(s)} y \bar{F}_{k}(y) \mathrm{d} y\right| \mathrm{d} s \\
& +\kappa_{k}^{2}\left(\lambda_{j}+\lambda\right) q_{k} \int_{t}^{T} \mathrm{e}^{2 r(T-s)}\left|\int_{a_{j}^{*, \mathfrak{n}}(s)}^{\tilde{a}_{j}^{*}(s)} y \bar{F}_{j}(y) \mathrm{d} y\right| \mathrm{d} s
\end{aligned}
$$




$$
\begin{aligned}
& +\lambda \kappa_{k} q_{k} \int_{t}^{T} \mathrm{e}^{2 r(T-s)}\left[\left|\left(\int_{a_{k}^{*, \mathfrak{n}}(s)}^{\tilde{a}_{k}^{*}(s)} \bar{F}_{k}(y) \mathrm{d} y\right)\left(\int_{0}^{\tilde{a}_{j}^{*}(s)} \bar{F}_{j}(y) \mathrm{d} y\right)\right|\right. \\
& \left.+\left|\left(\int_{0}^{a_{k}^{*, \mathfrak{n}}(s)} \bar{F}_{k}(y) \mathrm{d} y\right)\left(\int_{a_{j}^{*, \mathfrak{n}}(s)}^{\tilde{a}_{j}^{*}(s)} \bar{F}_{j}(y) \mathrm{d} y\right)\right|\right] \mathrm{d} s \\
& +\mid \lambda \mathfrak{n}^{\mathfrak{a}}(C(\mathfrak{n}))^{3} \kappa_{k}^{2} q_{k}^{2} \int_{t}^{T} \mathrm{e}^{3 r(T-s)}\left[\int_{0}^{a_{k}^{*, \mathfrak{n}}(s)} \bar{F}_{k}(y) \mathrm{d} y\right]\left[\int_{0}^{a_{j}^{*, \mathfrak{n}}(s)} y \bar{F}_{j}(y) \mathrm{d} y\right] \mathrm{d} s \\
& -\lambda \mathfrak{n}^{\mathfrak{a}}(C(\mathfrak{n}))^{3} \kappa_{k} q_{k}^{2} \int_{t}^{T} \mathrm{e}^{3 r(T-s)}\left[\int_{0}^{a_{k}^{*, \mathfrak{n}}(s)} y \bar{F}_{k}(y) \mathrm{d} y\right]\left[\int_{0}^{a_{j}^{*, \mathfrak{n}}(s)} \bar{F}_{j}(y) \mathrm{d} y\right] \mathrm{d} s \\
& +\lambda \mathfrak{n}^{\mathfrak{a}}(C(\mathfrak{n}))^{4} \kappa_{k}^{2} q_{k}^{3} \int_{t}^{T} \mathrm{e}^{4 r(T-s)}\left[\int_{0}^{a_{k}^{*, \mathfrak{n}}(s)} y \bar{F}_{k}(y) \mathrm{d} y\right]\left[\int_{0}^{a_{j}^{*, \mathfrak{n}}(s)} y \bar{F}_{j}(y) \mathrm{d} y\right] \mathrm{d} s \mid \\
& +O\left(\mathfrak{n}^{-\frac{\mathfrak{a}}{2}}\right)
\end{aligned}
$$

fro which the desired result (51) follows from the condition (53), together with the assumption that $C(\mathfrak{n})=O\left(\mathfrak{n}^{-\frac{\mathfrak{a}}{2}}\right)$ and that $\left\|\tilde{a}_{k}^{*}-a_{k}^{*, \mathfrak{n}}\right\|_{\infty}=O\left(\mathfrak{n}^{-\frac{\mathfrak{a}}{2}}\right)$, for $k=1,2$.

Finally, from the definitions of the objection function of insurer $k$, for $k=1,2, J_{k}^{\mathfrak{n}}$ in (6) (respectively, $\tilde{J}_{k}$ in (38)) and the corresponding value function $V^{k, \mathfrak{n}}$ in (9) (respectively, $\tilde{V}^{k}$ in (39)), we see that (51) is equivalent to

$$
\left\|J_{k}^{\mathfrak{n}}\left(., x_{k}, x_{j}, \pi_{k}^{*, \mathfrak{n}}, \pi_{j}^{*, \mathfrak{n}}\right)-\tilde{J}_{k}\left(., x_{k}, x_{j}, \tilde{\pi}_{k}^{*}, \tilde{\pi}_{j}^{*}\right)\right\|_{\infty}=O\left(\mathfrak{n}^{-\frac{\mathfrak{a}}{2}}\right) .
$$

By similar arguments, we can also deduce that

$$
\left\|\tilde{J}_{k}\left(., x_{k}, x_{j}, \tilde{\pi}_{k}^{*}, \tilde{\pi}_{j}^{*}\right)-J_{k}^{\mathfrak{n}}\left(., x_{k}, x_{j}, \tilde{\pi}_{k}^{*}, \tilde{\pi}_{j}^{*}\right)\right\|_{\infty}=O\left(\mathfrak{n}^{-\frac{\mathfrak{a}}{2}}\right),
$$

from which (52) follows by observing that

$$
\begin{aligned}
\left\|J_{k}^{\mathfrak{n}}\left(., x_{k}, x_{j}, \pi_{k}^{*, \mathfrak{n}}, \pi_{j}^{*, \mathfrak{n}}\right)-J_{k}^{\mathfrak{n}}\left(., x_{k}, x_{j}, \tilde{\pi}_{k}^{*}, \tilde{\pi}_{j}^{*}\right)\right\|_{\infty} \leq & \left\|J_{k}^{\mathfrak{n}}\left(., x_{k}, x_{j}, \pi_{k}^{*, \mathfrak{n}}, \pi_{j}^{*, \mathfrak{n}}\right)-\tilde{J}_{k}\left(., x_{k}, x_{j}, \tilde{\pi}_{k}^{*}, \tilde{\pi}_{j}^{*}\right)\right\|_{\infty} \\
& +\left\|\tilde{J}_{k}\left(., x_{k}, x_{j}, \tilde{\pi}_{k}^{*}, \tilde{\pi}_{j}^{*}\right)-J_{k}^{\mathfrak{n}}\left(., x_{k}, x_{j}, \tilde{\pi}_{k}^{*}, \tilde{\pi}_{j}^{*}\right)\right\|_{\infty} \\
= & O\left(\mathfrak{n}^{-\frac{\mathfrak{a}}{2}}\right) .
\end{aligned}
$$

Remark 6.3. For the case when $\mathfrak{a}=1$ and $C(\mathfrak{n})=\mathfrak{n}^{-\frac{1}{2}}$, we can recover the weak convergence result in Theorem 2.1 of Bai et al. (2013) that

$$
\hat{X}_{k}^{\pi_{k}^{\mathfrak{n}}} \Longrightarrow \hat{\mathcal{X}}_{k}^{\tilde{\pi}_{k}}, \quad \text { as } \quad \mathfrak{n} \rightarrow \infty
$$

Remark 6.4. In the proof of Theorem 6.2, under condition (49), (58) shows explicitly that the condition of Nash equilibrium under the general Poisson process, i.e. $\mathcal{Y}_{\mathfrak{n}}<1$, is satisfied automatically for sufficiently large $\mathfrak{n}$. 


\section{$7 \quad$ Numerical studies}

\begin{tabular}{|c|c|c|c|c|c|c|c|c|c|}
\hline \multicolumn{10}{|c|}{ Base parameters } \\
\hline$m$ & $r$ & $\alpha$ & $\delta$ & $\sigma$ & & $\rho_{S}$ & I & & $\lambda$ \\
\hline 1.5 & 0.05 & 2 & 0.3 & 1 & & $.3 / 0$. & 1 & 0 & 0.5 \\
\hline \multicolumn{4}{|c|}{ Insurer 1} & & \multicolumn{5}{|c|}{ Insurer 2} \\
\hline$\theta$ & $\lambda_{1}$ & $q_{1}$ & $\kappa_{1}$ & & $\theta_{2}$ & $\lambda_{2}$ & $q_{2}$ & $\kappa$ & \\
\hline 0. & 1 & 0.2 & 0.3 & & 0.8 & 4 & 0.9 & 0 . & 7 \\
\hline
\end{tabular}

Table 1: Model parameters

In this section, we shall illustrate the Nash equilibrium reinsurance and investment strategies under the CARA insurers in Sections 5 and 6 . In addition, we assume that $F_{1}(y)=1-\frac{1}{(1+y)^{3}}$ and $F_{2}(y)=1-\mathrm{e}^{-2 y}$, for $y \geq 0$. Unless otherwise stated, the following numerical illustrations are based on the model parameters as specified in Table 1.

\subsection{Equilibrium reinsurance strategies}
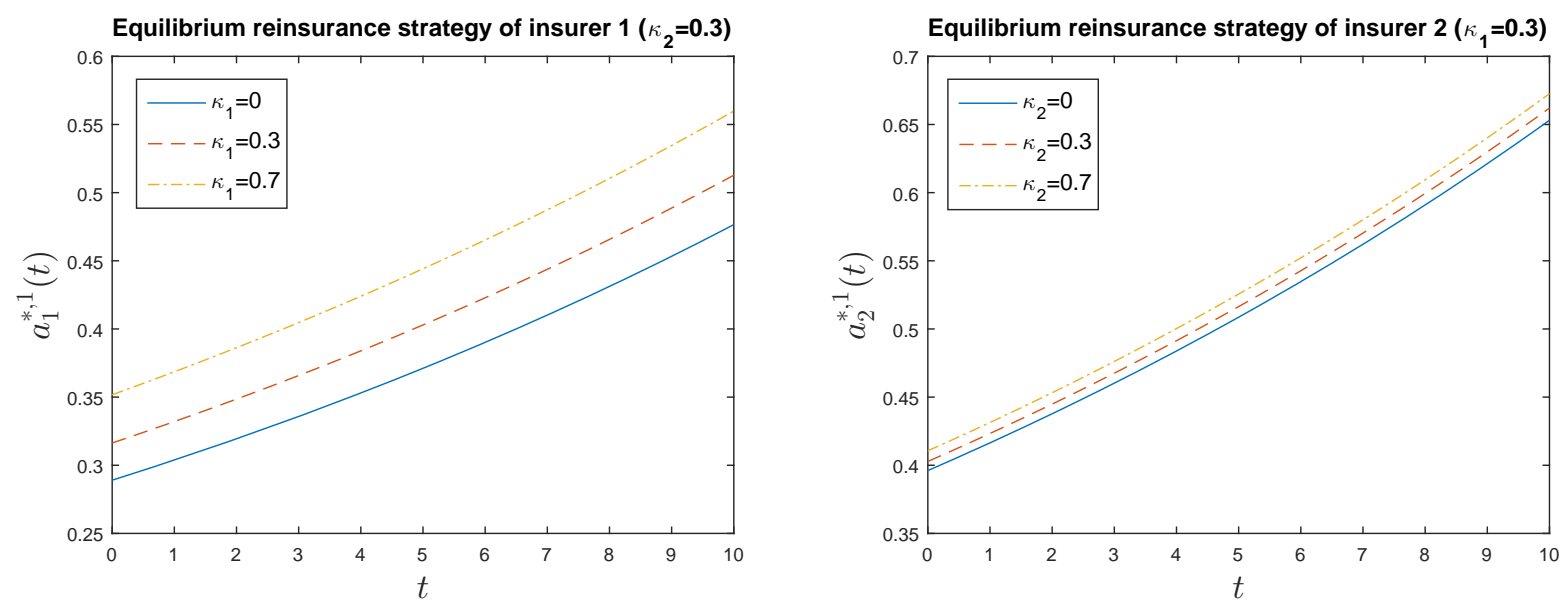

Figure 1: Effect of $\kappa_{k}$ on the equilibrium reinsurance strategy of insurer $k, a_{k}^{*, 1}$, for $k=1,2$

Figure 1 displays the effect of competition on the equilibrium reinsurance strategies under the Poisson model in Section 5 when $\mathfrak{n}=1$ and $C(\mathfrak{n}) \equiv 1$. Observe first that both insurers increase their respective retention levels $a_{k}^{*, 1}$, for $k=1,2$, at equilibrium as $t$ approaches to $T$. As the main objective of each insurer is to maximize the expected relative wealth at the terminal time $T$, each insurer wants to minimize the expenditure on the reinsurance protection by increasing his own retention level. The effect of competition is illustrated via the sensitivity parameter $\kappa_{k}$, for $k=1,2$. In Figure 1 , we see that the equilibrium retention level $a_{k}^{*, 1}$, for $k=1,2$, increases as $\kappa_{k}$ increases, with the absence of competition represented by the case when $\kappa_{k}=0$. This can be explained by the fact that the relative performance concern makes each insurer to be more risk-taking by increasing his respective retention levels as they are more concern with his expected terminal wealth relative to that of his competitors. This is 
also consistent with the proportional reinsurance protection game considered in Bensoussan et al. (2014). Finally, note that although both insurers increase their respective retention levels as each of their own $\kappa_{k}$ increases, increment in insurer 2's retention level is relatively small compared with that of insurer 1 . This is due to the fact that insurer 2 is more riskaverse than insurer 1 , ceteris paribus, i.e. $q_{2}=0.9>0.2=q_{1}$. Hence, even if insurer 2 is more concerned with his relative performance against that of insurer $1\left(\kappa_{2}=0.7>0.3=\kappa_{1}\right)$, insurer 2 is relatively risk-averse that he refrains himself from aggressively minimizing the reinsurance protection by increasing the retention level dramatically.
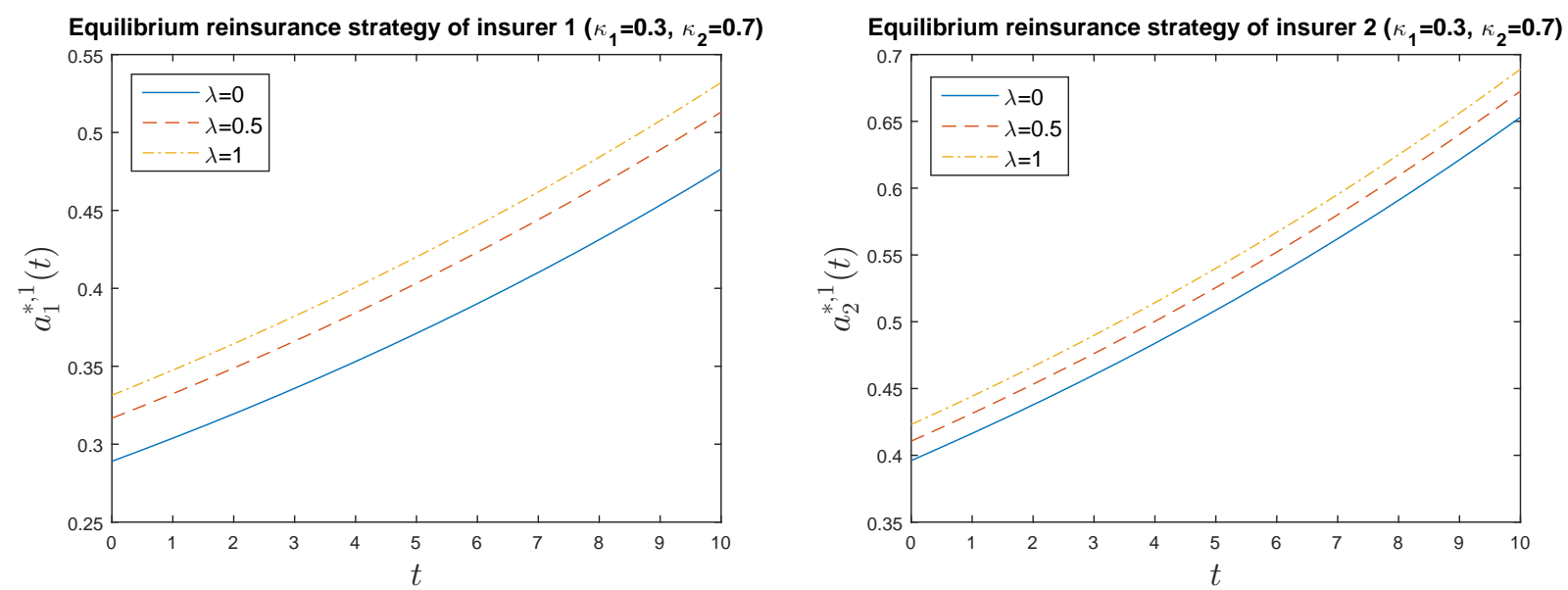

Figure 2: Effect of $\lambda$ on the equilibrium reinsurance strategy of insurer $k, a_{k}^{*, 1}$, for $k=1,2$

Figure 2 studies the effect of the systematic risk generated from the common intensity $\lambda$ of the Poisson risk model in (4) on the equilibrium reinsurance strategy $a_{k}^{*, 1}(t)$, for $k=1,2$ and $t \in[0, T]$. The case when both insurers have independent claim processes is represented by $\lambda=0$, under which we recover the classical optimal excess-of-loss retention level in the absence of competition. Indeed, when $\lambda=0$, the coupling effect in the integral-typed system (23) disappears, and we then have $a_{k}^{*, \mathfrak{n}}(t)=\frac{\mathrm{e}^{-r(T-t)}}{q_{k} C(\mathfrak{n})} \ln \left(1+\frac{\theta_{k}}{\mathfrak{n}^{\mathfrak{a}} C(\mathfrak{n})}\right)$, for $k=1,2, \mathfrak{n} \in \mathbb{N}$, and $\mathfrak{a} \geq 1$. In other words, when $\lambda=0$, the relative performance concern of each insurer becomes irrelevant in the context of reinsurance protection, as he would simply choose his optimal reinsurance protection as if there were no competition.

On the other hand, as $\lambda$ increases, the correlation between both insurers' claim risks increases. The prime objective of each insurer is to maximize his relative surplus relative to that of his competitor, and hence an increase in correlation of their claim risks minimizes the relative difference of their surpluses. As the arrival of common claim, measured by the intensity $\lambda$, affects both of them, there is certainly no reason to increase his excess-of-loss reinsurance expenditure with an increasing $\lambda$, for it would do nothing or even negatively to maximize his relative terminal surplus against his competitor. Instead, the best response of each insurer to the increase of $\lambda$ is then to decrease the corresponding expenditure on their excess-of-loss reinsurance, i.e. by increasing his retention level $a_{k}^{*, 1}(t)$, for $k=1,2$, so as to limit the potential of common loss. This effect is summarized in Figure 2. 

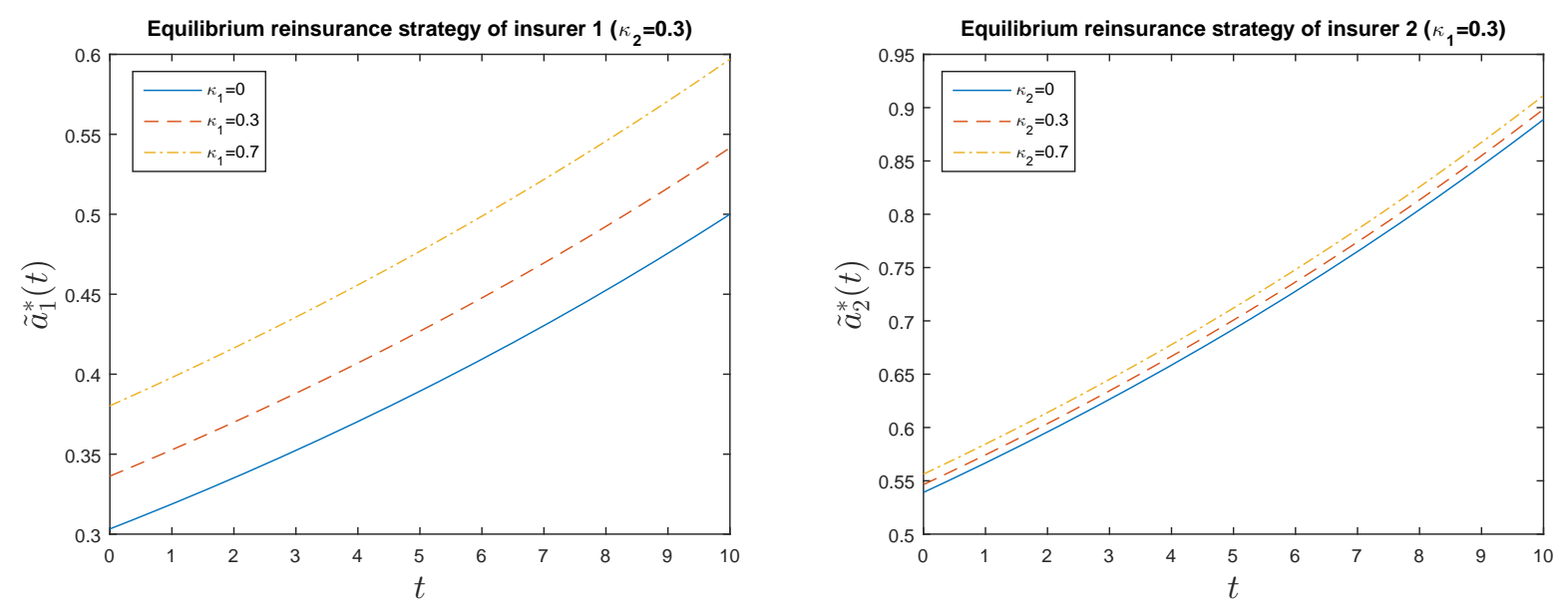

Figure 3: Effect of $\kappa_{k}$ on the equilibrium reinsurance strategy of insurer $k, \tilde{a}_{k}^{*}$ (Diffusionapproximated model), for $k=1,2$
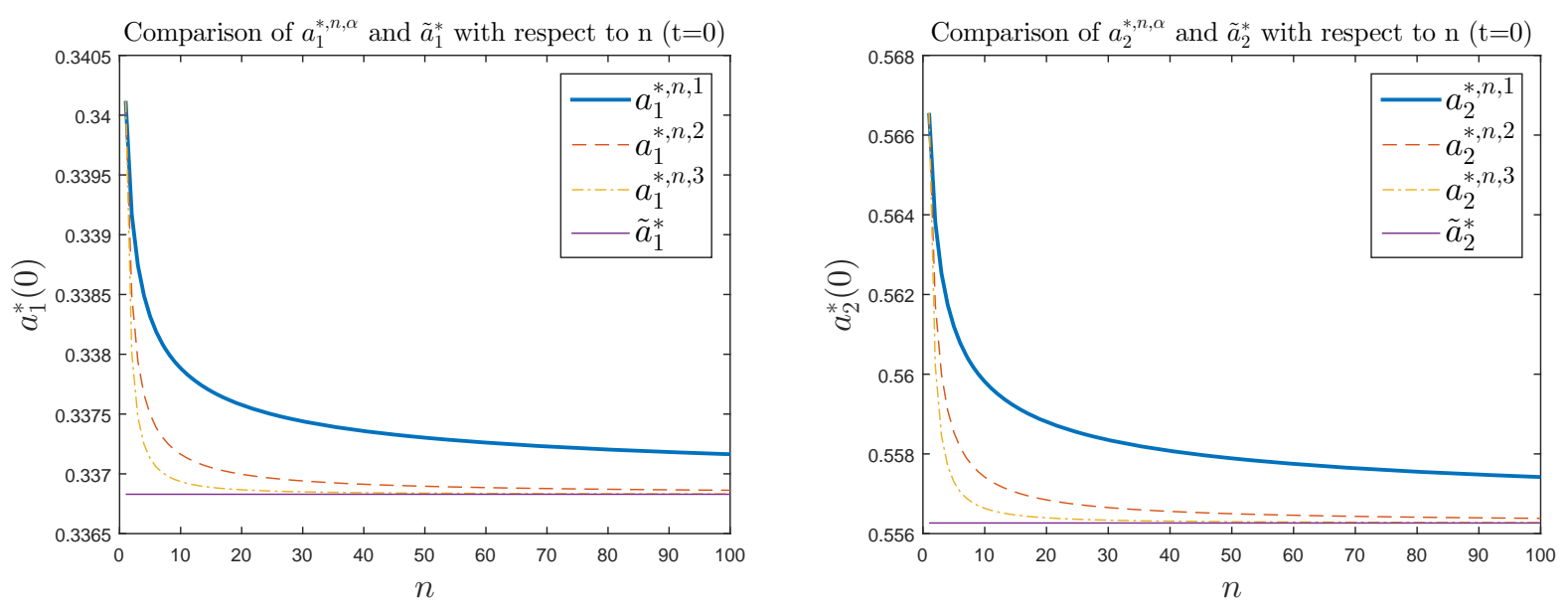

Figure 4: Convergence of $a_{k}^{*, \mathfrak{n}, \mathfrak{a}}(0)$ to $\tilde{a}_{k}^{*}(0)$ with respect to $\mathfrak{n}\left(\kappa_{1}=0.3, \kappa_{2}=0.7\right)$, for $k=1,2$ and $\mathfrak{a}=1,2,3$, at $t=0$.

Figure 3 captures the same effects of Figure 1 under the corresponding diffusion-approximated model in (37) with the model parameters in Table 1. Note that the patterns of the equilibrium retention levels, $\tilde{a}_{k}^{*}$, for $k=1,2$, are same as those in Figure 1, albeit with difference values. To understand the connection between Figures 1 and 3, we now turn to Figure 4. Figure 4 provides the graphical representation of the $\epsilon$-Nash equilibrium for the general Compound Poisson process in Theorem 6.2. More specifically, Figure 4 illustrates the convergence of Nash equilibrium under the compound Poisson model in (8) to that under the associated diffusionapproximated process in (37) with respect to $\mathfrak{n}$ under the case when $\kappa_{1}=0.3, \kappa_{2}=0.7$ and $t=0$, for $\mathfrak{a}=1,2,3$. Denote by $a_{k}^{*, \mathfrak{n}, \mathfrak{a}}$, for $k=1,2$, the corresponding equilibrium reinsurance strategy of insurer $k$ under the compound Poisson model in (8). As $\mathfrak{n}$ increases, we see that each equilibrium strategy $a_{k}^{*, \mathfrak{n}, \mathfrak{a}}$, for $\mathfrak{a}=1,2,3$, converges to the equilibrium strategy $\tilde{a}_{k}^{*}$, under the diffusion-approximated model in (37) with different rates. In this respect, parameter $\mathfrak{a}$ controls the speed of $a_{k}^{*, \mathfrak{n}, \mathfrak{a}}$ converging to $\tilde{a}_{k}^{*}$ with the increasing value of $\mathfrak{a}$ speeds up the corresponding convergence. More importantly, Figure 4 shows graphically that the Nash equilibrium strategies under the diffusion-approximated model, which admits high degree of 
tractability, can provide an accurate approximation to the Nash equilibrium strategies under the general Poisson model when the condition (49) in Theorem 6.2 is satisfied.

\subsection{Equilibrium investment strategies}
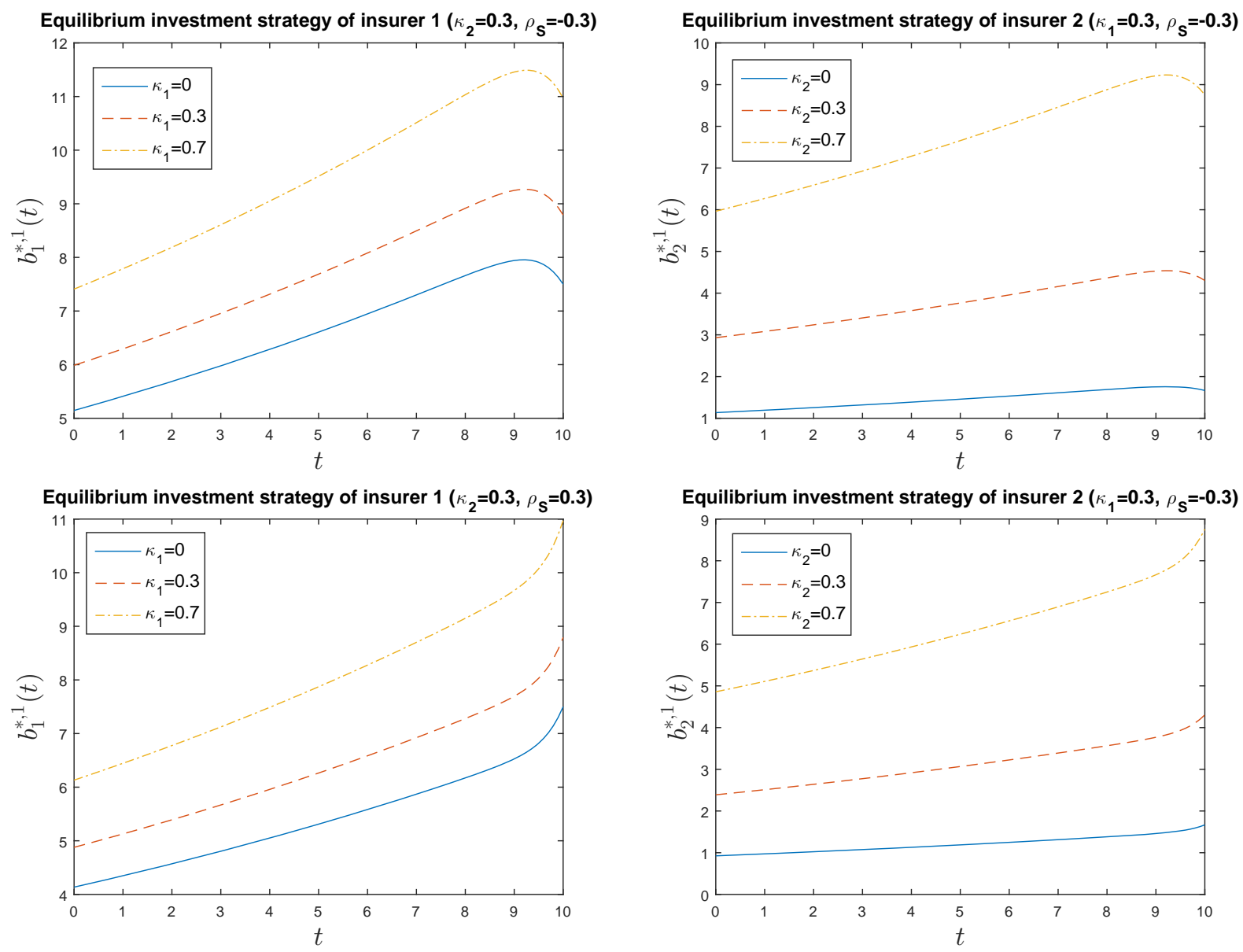

Figure 5: Effect of $\kappa_{k}$ on the equilibrium investment strategy of insurer $k, b_{k}^{*, 1}$, for $k=1,2$

We now proceed to investigate the equilibrium investment strategies, $b_{k}^{*, \mathfrak{n}}$, for $k=1,2$, of the two CARA insurers in Figure 5 when $\mathfrak{n}=1$ and $C(\mathfrak{n}) \equiv 1$. In contrast with the equilibrium strategies $a_{k}^{*, \mathfrak{n}}$, for $k=1,2$, which are monotonically increasing with respect to $t$, the change of the equilibrium investment strategy $b_{k}^{*, 1}$ with respect to $t$ depends on the sign of the correlation of between the risky asset $S$ and its volatility process $L$, i.e. $\rho_{S}$. When $\rho_{S}<0$, $b_{k}^{*, 1}$ in (24) shows that the term $\mathrm{e}^{-r(T-t)}$ is an increasing function of $t$, whereas $A(t)$ is a decreasing function of $t$. The resolution of this opposite effect is captured in the first row of Figure 5. On the other hand, when $\rho_{S}>0$, this opposite effect in $(24)$ disappears and $b_{k}^{*, 1}$ becomes monotonically increasing with respect to $t$, as appeared in the second row of Figure 5. Since both insurers share the same investment risk by investing in the same risky asset $S$, the sign of the correlation effect of the systematic risk from the investment can have a profound effect on the equilibrium investment of the insurers. 
In addition, Figure 5 also captures the effect of competition on the equilibrium investment strategies. Contrary to the case of the reinsurance protection, which is an expenditure, investment into the risky asset $S$ has a possibility of generating income and hence CARA insurers would increase their exposure to the risky asset $S$ as the time approaches to the maturity $T$. Moreover, the presence of competition, which is captured by the sensitivity parameter $\kappa_{k}$, for $k=1,2$, induces both CARA insurers to increase their exposure on the risky asset $S$. This is true for both cases when $\rho_{S}$ is positive or negative. This is also consistent with the equilibrium investment strategy under the regime-switching case in Bensoussan et al. (2014).
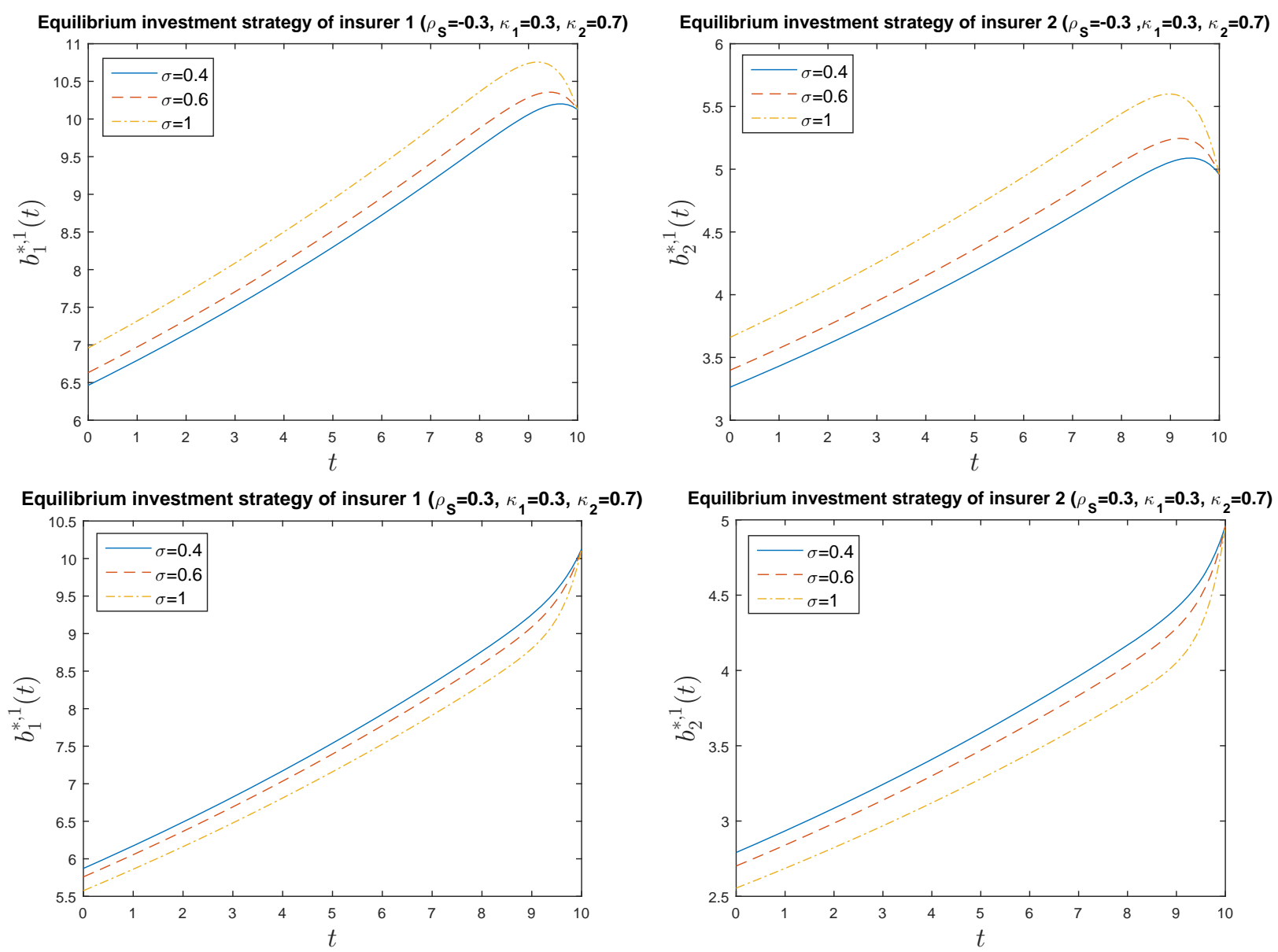

Figure 6: Effects of $\sigma$ and $\rho_{S}$ on the equilibrium investment strategy of insurer $k, b_{k}^{*, 1}$, for $k=1,2$

Figure 6 studies the impact of the volatility of the volatility process $L$, i.e. $\sigma$, on the equilibrium investment strategies with respect to the correlation $\rho_{S}$. When $\rho_{S}<0$, increase in $\sigma$ results in an increase in investment $b_{k}^{*, 1}$ at equilibrium, as shown in the first row of Figure 6. On the other hand, when $\rho_{S}>0$, increase in $\sigma$ leads to a decrease in the investment $b_{k}^{*, 1}$ at equilibrium, as appeared in the second row of Figure 6 . This can be explained by the fact that when $\rho_{S}<0$, the risky asset $S$ and its volatility process $L$ move in the opposite directions, implying that there is a compensating effect brought by the volatility process $L$ to the dynamics of the risky asset $S$. This in turn leads to a higher expected return of the 
risky asset $S$, see (3), and hence higher a $\sigma$ would result in an insurer investing more into the risky asset $S$, when $\rho_{S}<0$. On the other hand, when $\rho_{S}>0$, the risky asset $S$ and its volatility process move in the same direction, and the aforementioned compensating effect from the volatility process $L$ disappears. This means that, any adverse movement in the volatility process would lead to the erosion in the value of the risky asset, with the resulting effect being further pronounced by the increasing value of $\sigma$. This certainly leads to the reduction in investment into the risky asset $S$ as $\sigma$ increases, when $\rho_{S}>0$. It is worthy to mention that the effects in Figure 6 are strikingly different from the corresponding result in Bensoussan et al. (2014). In Bensoussan et al. (2014), the stochastic volatility effect is modeled by the regime-switching process that is independent of the process of the risky asset. Hence, as discussed in Bensoussan et al. (2014), increase in $\sigma$ would always lead to decrease in investment into the risky asset when $L_{t}=\sigma$, for all $t \geq 0$. In other words, the specification of the correlation factor plays a critical role in quantifying the systematic investment risks of two competing insurers in equilibrium.

Finally, note that the equilibrium investment strategies $b_{k}^{*, 1}$ for different $\sigma$ converge to a common value as $t$ approaches to $T$. To understand this, we revisit $b_{k}^{*, 1}$ in (24). When $t=T, A(T)=0$. Hence, $b_{k}^{*, \mathfrak{n}}(T)=\frac{1}{1-\kappa_{1} \kappa_{2}}\left[\frac{m}{q_{k}}+\frac{\kappa_{k} m}{q_{j}}\right]$, for $k \neq j \in\{1,2\}$, which is clearly independent of $\sigma$ and is the common value to which the equilibrium investment strategies $b_{k}^{*, 1}$ under different values of $\sigma$ converge when $t=T$.

\section{Conclusion}

In this paper, we study the impact of common systematic risks on the nonzero-sum reinsurance and investment game between two insurance companies under a general compound Poisson risk model. The first source of common systematic risks derives from the mutual dependency between two insurers, by modeling the surplus process of each insurance company with the correlated compound Poisson risk model. In addition, we consider the excess-of-loss reinsurance protection, instead of the proportional reinsurance protection, as the excess-ofloss reinsurance protection is of higher demand in reality. The consideration of the excessof-loss reinsurance proves to be more technically demanding to establish the resulting Nash equilibrium than the case of the proportional reinsurance protection, making the methodology employed in Bensoussan et al. (2014) not directly applicable in the present context. More specifically, the existence of the equilibrium excess-of-loss reinsurance strategies is equivalent to the solvability of the coupled non-linear integral-typed equations, as opposed to the solution of the system of linear equations in Bensoussan et al. (2014). Under some mild condition, we show that the equilibrium reinsurance and investment strategies uniquely exist, and that we show that this mild condition can be satisfied when the intensity of the systematic jump risk is relatively small in comparison with the intensities of the idiosyncratic jump risks of the insurers. Moreover, our numerical example illustrates that increasing the intensity of the systematic jump risks would lead to an increase the equilibrium retention level for each insurer, indicating the reduction in the demand of the reinsurance protection at equilibrium. This fits logically with our understanding on that systematic jump risk affects both insurers and hence increasing reinsurance protection would only lead to the erosion of the terminal relative surplus, and hence does not constitute the best response to each insurer. Finally, to connect the equilibrium results under the compound Poisson risk model to that 
under the diffusion-approximated model, we also provide the explicit equilibrium reinsuranceinvestment strategies under the diffusion-approximated model. More importantly, we show that the Nash equilibrium under the diffusion-approximated process is the $\epsilon$-Nash equilibrium for the general compound Poisson process.

The second source of systematic risk comes from investment on the same risky asset by the two insurers. We quantify the impact of volatility risk of the risky asset on the insurers' equilibrium investment strategies by modeling the risky asset with the popular Heston (1993) stochastic volatility model. We show that the correlation between the risky asset and its volatility process determines the directional behaviors of the investment strategies of the insurers at equilibrium with respect to the parameters of the volatility process. More specifically, we show that when the correlation between the risky asset and its volatility process is negative (respectively, positive), increasing the level of the volatility process would lead to an increase (respectively, decrease) in investment on that risky asset in equilibrium. This enriches the conclusion in Bensoussan et al. (2014) in which the equilibrium investment strategy of each insurer is always inversely proportional to the volatility, i.e. when the volatility process collapses into a constant. Our result also implies that modeling the correlation factor can have a significant impact on the dynamics of the equilibrium investment strategies.

There are several extensions of the present work that can be considered. First extension is to enrich the nonzero-sum game framework to other utility functions. We choose to study the exponential utility for the sake of tractability and comparative static analyses. For other utility functions, closed-form solution to the Nash equilibrium may not be available and reliable numerical scheme is necessary to solve the coupled system of PDEs in Theorem 4.2. Secondly, a consideration of the consumption strategies, in addition to the investment and reinsurance strategies, can also be interesting as it allows us to investigate intertemporal consumption behaviors of the insurers under competition. Finally, this paper shows explicitly the nature of the convergence of the Nash equilibrium under the compound Poisson model to that under the diffusion-approximated analogue. In this respect, it is also well-known that a compound Poisson process can be used to approximate wide class of Lévy processes, e.g. $\alpha$-stable process, (see, for example, Corollary 8.8 of Sato (1999)) in addition to the diffusion process. Since we show that the Nash equilibrium under the general compound Poisson model uniquely exists with a closed-form expression, it is interesting to see if this result can be used to study the Nash equilibrium under other Lévy processes that lacks analytical tractability. We leave these suggestions for future research.

\section{Acknowledgement}

Authors would like to thank anonymous referees for their insightful comments. Chi Chung Siu acknowledges the financial support from 2014 UTS Business Research Grant from the University of Technology, Sydney. Phillip Yam acknowledges the financial supports from the Hong Kong RGC GRF 404012 for the project titled "Advanced Topics In Multivariate Risk Management In Finance And Insurance and Direct Grant for Research with Project Code 4053141 offered by CUHK. Phillip Yam also expresses his sincere gratitude to both the Hausdorff Center for Mathematics at the University of Bonn for their hospitality during the preparation of the present work. Hailiang Yang acknowledges support from the Research Grants Council of the Hong Kong Special Administrative Region (project No. HKU 705313P) 
and a Society of Actuaries Centers of Actuarial Excellence Research Grant. Hui Zhao acknowledges support from the National Science Foundation of China (Grant No. 17301816).

\section{A Proof of the Verification Theorem for $V^{k, \mathfrak{n}}$ in Theo- rems 5.1 and $\tilde{V}^{k}$ in 6.1}

Since the proof of the verification for Theorem 6.1 is analogous to that of Theorem 5.1, we shall only provide the verification proof for Theorem 5.1 below. In light of Kraft (2005), Taskar and Zeng (2013), and Zhao et al. (2013), we begin with the following lemma.

Lemma A.1. Let $Q=[0, \infty) \times[0, \infty)$. Take a sequence of bounded open sets, $Q_{1}, Q_{2}, Q_{3}, \ldots$ with $Q_{i} \subset Q_{i+1} \subset Q$, for $i \in \mathbb{N}$, and $Q=\cup_{i \in \mathbb{N}} Q_{i}$. Denote the exit time of $\left(\hat{X}_{k}^{\pi_{k}^{*, n}}(t), L(t)\right)$, for $k=1,2$, from $Q_{i}$ by $\tau_{i}$.

Let

$$
W^{k, \mathfrak{n}}(t, x, l) \triangleq-\frac{1}{q_{k}} \exp \left\{-q_{k}\left[x \mathrm{e}^{r(T-t)}+f^{k, \mathfrak{n}}(t)\right]-A(t) l-B(t)\right\},
$$

where $f^{k, \mathfrak{n}}, A$, and $B$, are of the forms in (20), (21), and (22), respectively. Then

$$
-\infty<\mathbb{E}\left[W^{k, \mathfrak{n}}\left(\tau_{i} \wedge T, \hat{X}_{k}^{\pi_{k}^{*, \mathfrak{n}}}\left(\tau_{i} \wedge T\right), L\left(\tau_{i} \wedge T\right)\right) \mid \hat{X}_{k}^{\pi_{k}^{*, \mathfrak{n}}}(t)=x, L(t)=l\right]<0,
$$

for all $i \in \mathbb{N}$.

Proof. From (8), $\hat{X}_{k}^{\pi_{k}^{*, \mathfrak{n}}}$ at equilibrium admits the following form:

$$
\begin{aligned}
\hat{X}_{k}^{\pi_{k}^{*, \mathfrak{n}}}(t)= & \mathrm{e}^{r t}\left\{\hat{x}_{k}+\int_{0}^{t} \mathrm{e}^{-r s}\left[\left(\lambda_{k}+\lambda\right)\left[\left(\theta_{k}+\mathfrak{n}^{\mathfrak{a}} C(\mathfrak{n})\right) \mu_{k}\left(a_{k}^{*, \mathfrak{n}}(s)\right)+\left(\eta_{k}-\theta_{k}\right) \bar{\mu}_{k}\right]\right.\right. \\
& \left.-\kappa_{k}\left(\lambda_{j}+\lambda\right)\left[\left(\theta_{j}+\mathfrak{n}^{\mathfrak{a}} C(\mathfrak{n})\right) \mu\left(a_{j}^{*, \mathfrak{n}}(s)\right)+\left(\eta_{j}-\theta_{j}\right) \bar{\mu}_{j}\right]\right] \mathrm{d} s \\
& +\int_{0}^{t} \mathrm{e}^{-r s} m\left[b_{k}^{*, \mathfrak{n}}(s)-\kappa_{k} b_{j}^{*, \mathfrak{n}}(s)\right] L(s) \mathrm{d} s+\int_{0}^{t} \mathrm{e}^{-r s}\left[b_{k}^{*, \mathfrak{n}}(s)-\kappa_{k} b_{j}^{*, \mathfrak{n}}(s)\right] \sqrt{L(s)} \mathrm{d} B_{S}(s) \\
& -\int_{0}^{t} \mathrm{e}^{-r s} C(\mathfrak{n})\left(Z^{k} \wedge a_{k}^{*, \mathfrak{n}}(s)\right) \mathrm{d}\left(N_{k}\left(\mathfrak{n}^{\mathfrak{a}} s\right)+N\left(\mathfrak{n}^{\mathfrak{a}} s\right)\right) \\
& \left.+\kappa_{k} \int_{0}^{t} \mathrm{e}^{-r s} C(\mathfrak{n})\left(Z^{j} \wedge a_{j}^{*, \mathfrak{n}}(s)\right) \mathrm{d}\left(N_{j}\left(\mathfrak{n}^{\mathfrak{a}} s\right)+N\left(\mathfrak{n}^{\mathfrak{a}} s\right)\right)\right\} .
\end{aligned}
$$

Since $\mathrm{d} B_{S}(s)=\rho_{S} \mathrm{~d} B_{L}(s)+\sqrt{1-\rho_{S}^{2}} \mathrm{~d} \bar{B}(s)$, where $d\left\langle B_{L}(s), \bar{B}(s)\right\rangle=0$, substituting (62) into the equilibrium value function in (19), we have

$$
\begin{aligned}
W^{k, \mathfrak{n}}\left(t, \hat{X}_{k}^{\pi_{k}^{*, \mathfrak{n}}}(t), L(t)\right)= & -\frac{1}{q_{k}} \exp \left\{-q_{k} \mathrm{e}^{r T} \hat{x}_{k}-q_{k} f^{k, \mathfrak{n}}(t)-B(t)\right. \\
& -q_{k} \int_{0}^{t} \mathrm{e}^{r(T-s)}\left[\left(\lambda_{k}+\lambda\right)\left[\left(\theta_{k}+\mathfrak{n}^{\mathfrak{a}} C(\mathfrak{n})\right) \mu_{k}\left(a_{k}^{*, \mathfrak{n}}(s)\right)+\left(\eta_{k}-\theta_{k}\right) \bar{\mu}_{k}\right]\right.
\end{aligned}
$$




$$
\begin{aligned}
& \left.\left.-\kappa_{k}\left(\lambda_{j}+\lambda\right)\left[\left(\theta_{j}+\mathfrak{n}^{\mathfrak{a}} C(\mathfrak{n})\right) \mu\left(a_{j}^{*, \mathfrak{n}}(s)\right)+\left(\eta_{j}-\theta_{j}\right) \bar{\mu}_{j}\right]\right] \mathrm{d} s\right\} \\
& \cdot \exp \left\{-q_{k} \int_{0}^{t} \mathrm{e}^{r(T-s)} m\left[b_{k}^{*, \mathfrak{n}}(s)-\kappa_{k} b_{j}^{*, \mathfrak{n}}(s)\right] L(s) \mathrm{d} s\right. \\
& +\frac{q_{k}^{2}\left(1-\rho_{S}^{2}\right)}{2} \int_{0}^{t} \mathrm{e}^{2 r(T-s)}\left[b_{k}^{*, \mathfrak{n}}(s)-\kappa_{k} b_{j}^{*, \mathfrak{n}}(s)\right]^{2} L(s) \mathrm{d} s \\
& \left.-q_{k} \rho_{S} \int_{0}^{t} \mathrm{e}^{r(T-s)}\left[b_{k}^{*, \mathfrak{n}}(s)-\kappa_{k} b_{j}^{*, \mathfrak{n}}(s)\right] \sqrt{L(s)} \mathrm{d} B_{L}(s)-A(t) L(t)\right\} \\
& \cdot \exp \left\{-\frac{q_{k}^{2}\left(1-\rho_{S}^{2}\right)}{2} \int_{0}^{t} \mathrm{e}^{2 r(T-s)}\left[b_{k}^{*, \mathfrak{n}}(s)-\kappa_{k} b_{j}^{*, \mathfrak{n}}(s)\right]^{2} L(s) \mathrm{d} s\right. \\
& \left.-q_{k} \sqrt{1-\rho_{S}^{2}} \int_{0}^{t} \mathrm{e}^{r(T-s)}\left[b_{k}^{*, \mathfrak{n}}(s)-\kappa_{k} b_{j}^{*, \mathfrak{n}}(s)\right] \sqrt{L(s)} \mathrm{d} \bar{B}(s)\right\} \\
& \cdot \exp \left\{q_{k} C(\mathfrak{n}) \int_{0}^{t} \mathrm{e}^{r(T-s)}\left(Z^{k} \wedge a_{k}^{*, \mathfrak{n}}(s)\right) \mathrm{d}\left(N_{k}\left(\mathfrak{n}^{\mathfrak{a}} s\right)+N\left(\mathfrak{n}^{\mathfrak{a}} s\right)\right)\right. \\
& \left.-\kappa_{k} q_{k} C(\mathfrak{n}) \int_{0}^{t} \mathrm{e}^{r(T-s)}\left(Z^{j} \wedge a_{j}^{*, \mathfrak{n}}(s)\right) \mathrm{d}\left(N_{j}\left(\mathfrak{n}^{\mathfrak{a}} s\right)+N\left(\mathfrak{n}^{\mathfrak{a}} s\right)\right)\right\} \\
& \triangleq \mathbb{G}_{1}^{k}(t) \mathbb{G}_{2}^{k}(t) \mathbb{G}_{3}^{k}(t) \mathbb{G}_{4}^{k}(t),
\end{aligned}
$$

where, for $k=1,2$,

$$
\begin{aligned}
\mathbb{G}_{1}^{k}(t) \triangleq & \frac{1}{q_{k}} \exp \left\{-q_{k} \mathrm{e}^{r T} \hat{x}_{k}-q_{k} f^{k, \mathfrak{n}}(t)-B(t)\right. \\
& -q_{k} \int_{0}^{t} \mathrm{e}^{r(T-s)}\left[\left(\lambda_{k}+\lambda\right)\left[\left(\theta_{k}+\mathfrak{n}^{\mathfrak{a}} C(\mathfrak{n})\right) \mu_{k}\left(a_{k}^{*, \mathfrak{n}}(s)\right)+\left(\eta_{k}-\theta_{k}\right) \bar{\mu}_{k}\right]\right. \\
& \left.\left.-\kappa_{k}\left(\lambda_{j}+\lambda\right)\left[\left(\theta_{j}+\mathfrak{n}^{\mathfrak{a}} C(\mathfrak{n})\right) \mu\left(a_{j}^{*, \mathfrak{n}}(s)\right)+\left(\eta_{j}-\theta_{j}\right) \bar{\mu}_{j}\right]\right] \mathrm{d} s\right\} \\
\mathbb{G}_{2}^{k}(t) \triangleq & -\exp \left\{\int_{0}^{t} \mathrm{f}_{1}^{k}(s) L(s) \mathrm{d} s+\int_{0}^{t} \mathrm{f}_{2}^{k}(s) \sqrt{L(s)} \mathrm{d} B_{L}(s)+\mathrm{f}_{3}^{k}(t) L(t)\right\} \\
\mathbb{G}_{3}^{k}(t) \triangleq & \exp \left\{-\frac{q_{k}^{2}\left(1-\rho_{S}^{2}\right)}{2} \int_{0}^{t} \mathrm{e}^{2 r(T-s)}\left[b_{k}^{*, \mathfrak{n}}(s)-\kappa_{k} b_{j}^{*, \mathfrak{n}}(s)\right]^{2} L(s) \mathrm{d} s\right. \\
& \left.-q_{k} \sqrt{1-\rho_{S}^{2}} \int_{0}^{t} \mathrm{e}^{r(T-s)}\left[b_{k}^{*, \mathfrak{n}}(s)-\kappa_{k} b_{j}^{*, \mathfrak{n}}(s)\right] \sqrt{L(s)} \mathrm{d} \bar{B}(s)\right\} \\
\triangleq & \quad \exp \left\{q_{k} C(\mathfrak{n}) \int_{0}^{t} \mathrm{e}^{r(T-s)}\left(Z^{k} \wedge a_{k}^{*, \mathfrak{n}}(s)\right) \mathrm{d}\left(N_{k}\left(\mathfrak{n}^{\mathfrak{a}} s\right)+N\left(\mathfrak{n}^{\mathfrak{a}} s\right)\right)\right. \\
& \left.-\kappa_{k} q_{k} C(\mathfrak{n}) \int_{0}^{t} \mathrm{e}^{r(T-s)}\left(Z^{j} \wedge a_{j}^{*, \mathfrak{n}}(s)\right) \mathrm{d}\left(N_{j}\left(\mathfrak{n}^{\mathfrak{a}} s\right)+N\left(\mathfrak{n}^{\mathfrak{a}} s\right)\right)\right\}
\end{aligned}
$$


with

$$
\begin{aligned}
& \mathrm{f}_{1}^{k}(t) \triangleq-q_{k} \mathrm{e}^{r(T-t)} m\left[b_{k}^{*, \mathfrak{n}}(t)-\kappa_{k} b_{j}^{*, \mathfrak{n}}(t)\right]+\frac{q_{k}^{2}\left(1-\rho_{S}^{2}\right)}{2} \mathrm{e}^{2 r(T-t)}\left[b_{k}^{*, \mathfrak{n}}(t)-\kappa_{k} b_{j}^{*, \mathfrak{n}}(t)\right]^{2}, \\
& \mathrm{f}_{2}^{k}(t) \triangleq-q_{k} \rho_{S} \mathrm{e}^{r(T-t)}\left[b_{k}^{*, \mathfrak{n}}(t)-\kappa_{k} b_{j}^{*, \mathfrak{n}}(t)\right], \\
& \mathrm{f}_{3}^{k}(t) \triangleq-A(t),
\end{aligned}
$$

for $0 \leq t \leq T$.

Observe first that due to the uniform-boundedness of $a_{k}^{*, \mathfrak{n}}$, for $k=1,2 . \mathbb{G}_{1}^{k}(t)$ is uniformly bounded and therefore it follows that $0<\sup _{t \in[0, T]} \mathbb{G}_{1}^{k}(t)<\infty$.

Applying Itô's lemma to $\mathbb{G}_{2}^{k}(t)$, together with the dynamics of $L$ in (3), gives

$$
\left\{\begin{aligned}
\mathrm{d} \mathbb{G}_{2}^{k}(t)= & \mathbb{G}_{2}^{k}(t)\left\{\alpha \delta \mathrm{f}_{3}^{k}(t)+L(t)\left[\mathrm{f}_{1}^{k}(t)+\dot{f}_{3}^{k}(t)-\alpha \mathrm{f}_{3}^{k}(t)+\frac{1}{2}\left(\sigma \mathrm{f}_{3}^{k}(t)+\mathrm{f}_{2}^{k}(t)\right)^{2}\right]\right\} \mathrm{d} t \\
& +\mathbb{G}_{2}^{k}(t) \sqrt{L(t)}\left[\sigma \mathrm{f}_{3}^{k}(t)+\mathrm{f}_{2}^{k}(t)\right] \mathrm{d} B_{L}(t), \\
\mathbb{G}_{2}^{k}(0)= & -\exp \left(\mathrm{f}_{3}^{k}(0) L(0)\right) .
\end{aligned}\right.
$$

Since $b_{k}^{*, \mathfrak{n}}$ admits the form in (24) and $A$ satisfies the ODE in (30), direct calculation shows that

$$
\mathrm{f}_{1}^{k}(s)+\dot{\mathrm{f}}_{3}^{k}(s)-\alpha \mathrm{f}_{3}^{k}(s)+\frac{1}{2}\left(\sigma \mathrm{f}_{3}^{k}(s)+\mathrm{f}_{2}^{k}(s)\right)^{2}=0 ;
$$

and hence (64) now becomes

$$
\begin{aligned}
\mathbb{G}_{2}^{k}(t)= & -\exp \left(\mathrm{f}_{3}^{k}(0) L(0)+\int_{0}^{t} \alpha \delta \mathrm{f}_{3}^{k}(s) \mathrm{d} s\right) \\
& \cdot \exp \left\{-\frac{1}{2} \int_{0}^{t}\left(\sigma \mathrm{f}_{3}^{k}(s)+\mathrm{f}_{2}^{k}(s)\right)^{2} L(s) \mathrm{d} s+\int_{0}^{t} \sqrt{L(s)}\left[\sigma \mathrm{f}_{3}^{k}(s)+\mathrm{f}_{2}^{k}(s)\right] \mathrm{d} B_{L}(s)\right\} \\
\geq & -\exp \left\{\mathrm{f}_{3}^{k}(0) L(0)-\frac{1}{2} \int_{0}^{t}\left(\sigma \mathrm{f}_{3}^{k}(s)+\mathrm{f}_{2}^{k}(s)\right)^{2} L(s) \mathrm{d} s\right. \\
& \left.+\int_{0}^{t} \sqrt{L(s)}\left[\sigma \mathrm{f}_{3}^{k}(s)+\mathrm{f}_{2}^{k}(s)\right] \mathrm{d} B_{L}(s)\right\},
\end{aligned}
$$

where second inequality $A(t) \geq 0$, for all $t \in[0, T]$ and $\alpha, \delta \geq 0$, which implies that $\alpha \delta \mathrm{f}_{3}^{k}(t) \leq$ 0 , for all $t \in[0, T]$.

Finally, from Lemma 5.3, for each $\mathfrak{n} \in \mathbb{N}$ and $\mathfrak{a} \geq 1, a_{k}^{*, \mathfrak{n}}$, for $k=1,2$, is uniformly bounded. Together with the well-known fact that Poisson process has almost-surely finite number of jumps for $t \in[0, T]$, it follows readily that for $k=1,2, \mathfrak{n} \in \mathbb{N}$, and $\mathfrak{a} \geq 1$,

$$
0<\mathbb{E}\left[\mathbb{G}_{4}^{k}(t)\right] \leq \sup _{t \in[0, T]} \mathbb{E}\left[\mathbb{G}_{4}^{k}(t)\right]<\infty .
$$

Denote

$$
\begin{aligned}
\mathbb{L}^{k}(t) \triangleq & -\exp \left\{\mathrm{f}_{3}^{k}(0) L(0)-\frac{1}{2} \int_{0}^{t}\left(\sigma \mathrm{f}_{3}^{k}(s)+\mathrm{f}_{2}^{k}(s)\right)^{2} L(s) \mathrm{d} s\right. \\
& \left.+\int_{0}^{t} \sqrt{L(s)}\left[\sigma \mathrm{f}_{3}^{k}(s)+\mathrm{f}_{2}^{k}(s)\right] \mathrm{d} B_{L}(s)\right\} \mathbb{G}_{3}^{k}(t),
\end{aligned}
$$


and note that since $\mathbb{L}^{k}(t) \leq 0$, for all $t \in[0, T]$, the local martingale $\mathbb{L}^{k}$ is a submartingale, i.e

$$
\mathbb{E}\left[\mathbb{L}^{k}(t)\right] \geq \mathbb{L}^{k}(0)=-\exp \left\{\mathrm{f}_{3}^{k}(0) L(0)\right\},
$$

for all $t \in[0, T]$. Therefore, taking expectation on (63) yields

$$
\begin{aligned}
0 & >\mathbb{E}\left[W^{k, \mathfrak{n}}\left(t, \hat{X}_{k}^{\pi_{k}^{*, \mathfrak{n}}}(t), L(t)\right)\right]=\mathbb{E}\left[\mathbb{G}_{1}^{k}(t) \mathbb{G}_{2}^{k}(t) \mathbb{G}_{3}^{k}(t) \mathbb{G}_{4}^{k}(t)\right] \\
& \geq\left(\sup _{t \in[0, T]} \mathbb{G}_{1}^{k}(t)\right) \mathbb{E}\left[\mathbb{L}^{k}(t) \mathbb{G}_{4}^{k}(t)\right] \\
& =\left(\sup _{t \in[0, T]} \mathbb{G}_{1}^{k}(t)\right) \mathbb{E}\left[\mathbb{L}^{k}(t)\right] \mathbb{E}\left[\mathbb{G}_{4}^{k}(t)\right] \\
\geq & -\left(\sup _{t \in[0, T]} \mathbb{G}_{1}^{k}(t)\right) \exp \left(\mathrm{f}_{3}^{k}(0) L(0)\right) \mathbb{E}\left[\mathbb{G}_{4}^{k}(t)\right] \\
\geq & -\left(\sup _{t \in[0, T]} \mathbb{G}_{1}^{k}(t)\right) \exp \left(\mathrm{f}_{3}^{k}(0) L(0)\right)\left(\sup _{t \in[0, T]} \mathbb{E}\left[\mathbb{G}_{4}^{k}(t)\right]\right)>-\infty,
\end{aligned}
$$

where third inequality follows from $\sup _{t \in[0, T]} \mathbb{G}_{1}^{k}(t)<\infty$; the fourth equality follows from the fact that $\mathbb{L}^{k}$ and $\mathbb{G}_{4}^{k}$ are mutually independent; the fifth inequality follows from (67); and the sixth inequality follows from (66). Since (68) is independent of $t \in[0, T]$, the result now readily follows.

Proof. (Proof of the verification theorem in Theorem 5.1)

To verify that $W^{k, \mathfrak{n}}$ in (61) is the optimal value function of insurer $k$, for $k=1,2$, we need to establish (12) and (15) in Theorem 4.1. (12) follows from the arguments in the proof of Theorem 4.1 in Taskar and Zeng (2013) and the proof of Theorem 3.2 in Zhao et al. (2013), and we shall omit it here for brevity ${ }^{3}$. It remains to establish (15).

Denote $Q \triangleq[0, \infty) \times[0, \infty)$. Take a sequence of bounded open sets, $Q_{1}, Q_{2}, Q_{3}, \ldots$ with $Q_{i} \subset Q_{i+1} \subset Q$, for $i \in \mathbb{N}$, and $Q=\cup_{i \in \mathbb{N}} Q_{i}$. Denote the exit time of $\left(\hat{X}_{k}^{\pi_{k}^{*, n}}(t), L(t)\right)$, for $k=1,2$, from $Q_{i}$ by $\tau_{i}$. Then, it follows readily that $\tau_{i} \wedge T \rightarrow T$, as $i \rightarrow \infty$.

\footnotetext{
${ }^{3}$ Alternatively, for the case of CARA insurers, one can first construct a sequence of bounded controls $\pi_{k, l}^{\mathfrak{n}} \triangleq\left(a_{k}^{\mathfrak{n}} \wedge l \vee(-l), b_{k}^{\mathfrak{n}} \wedge l \vee(-l)\right)$, for $k=1,2, l \in \mathbb{N}$, such that $\pi_{k, l}^{\mathfrak{n}} \rightarrow \pi_{k}^{\mathfrak{n}}$ pointwisely, as $l \rightarrow \infty$. Secondly, for an arbitrary family of stopping times $\left\{\tau_{i}\right\}_{i \in \mathbb{N}}$, by invoking Lemma 4.3 in Taskar and Zeng (2013), one can follow the proof of Lemma A.1 to establish that, for each $l \in \mathbb{N}, W^{k, \mathfrak{n}}\left(\tau_{i} \wedge T, \hat{X}_{k}^{\pi_{k, l}^{\mathfrak{n}}}\left(\tau_{i} \wedge T\right), L\left(\tau_{i} \wedge T\right)\right)$ is uniformly integrable. Thirdly, using the Burkholder-Davis-Gundy inequalities along with some algebraic manipulations, as in the case of Ren (2008), one can show that a priori estimate for the error term $\mathbb{E}\left[\left(W^{k, \mathfrak{n}}\left(\tau_{i} \wedge T, \hat{X}_{k}^{\pi_{k, l}^{\mathfrak{n}}}\left(\tau_{i} \wedge T\right), L\left(\tau_{i} \wedge T\right)\right)-W^{k, \mathfrak{n}}\left(\tau_{i} \wedge T, \hat{X}_{k}^{\pi_{k}^{\mathfrak{n}}}\left(\tau_{i} \wedge T\right), L\left(\tau_{i} \wedge T\right)\right)\right)^{2}\right]$ is quite independent of $l$ and $\tau_{i}$. Combining these aforementioned results, one can readily deduce that $W^{k, \mathfrak{n}}\left(\tau_{i} \wedge T, \hat{X}_{k}^{\pi_{k}^{\mathfrak{n}}}\left(\tau_{i} \wedge T\right), L\left(\tau_{i} \wedge T\right)\right)$ is also uniformly integrable. With this uniform integrability condition established for $\pi_{k}^{\mathfrak{n}},(12)$ readily follows by interchanging the limit with the expectation operator, as in the case of proving (15).
} 
Since $\pi_{k}^{*, \mathfrak{n}}=\left(a_{k}^{*, \mathfrak{n}}, b_{k}^{*, \mathfrak{n}}\right) \in \Pi_{k}$, which is admissible, applying Itô's Lemma on $W^{k, \mathfrak{n}}$ and taking conditional expectation on $\left\{\hat{X}_{k}^{\pi_{k}^{*, n}}(t)=x, L(t)=l\right\}$ yields

$$
\begin{aligned}
& \mathbb{E}\left[W^{k, \mathfrak{n}}\left(\tau_{i} \wedge T, \hat{X}_{k}^{\pi_{k}^{*, \mathfrak{n}}}\left(\tau_{i} \wedge T\right), L\left(\tau_{i} \wedge T\right)\right) \mid \hat{X}_{k}^{\pi_{k}^{*, \mathfrak{n}}}(t)=x, L(t)=l\right] \\
= & W^{k, \mathfrak{n}}(t, x, l)+\mathbb{E}\left[\int_{t}^{\tau_{i} \wedge T}\left(W_{s}^{k, \mathfrak{n}}+\mathcal{L}^{\pi_{k}^{*, \mathfrak{n}}} W^{k, \mathfrak{n}}\left(s, \hat{X}_{k}^{\pi_{k}^{*, \mathfrak{n}}}(s), L(s)\right)\right) \mathrm{d} s \mid \hat{X}_{k}^{\pi_{k}^{*, \mathfrak{n}}}(t)=x, L(t)=l\right] \\
= & W^{k, \mathfrak{n}}(t, x, l),
\end{aligned}
$$

where the second equality follows from the fact that $W^{k, \mathfrak{n}}$ satisfies the HJB equation in (11) and $a_{k}^{*, \mathfrak{n}}, b_{k}^{*, \mathfrak{n}}$ are the solutions to (13) and (14), respectively.

Applying Lemma A.1, it follows that $W^{k, \mathfrak{n}}\left(\tau_{i} \wedge T, \hat{X}_{k}^{\pi_{k}^{*, \mathfrak{n}}}\left(\tau_{i} \wedge T\right), L\left(\tau_{i} \wedge T\right)\right)$, for $i \in \mathbb{N}$, is uniformly integrable. Hence, taking limit with respect to $i \in \mathbb{N}$ on both sides of (69), we then have

$$
\begin{aligned}
& \mathbb{E}\left[U^{k}\left(\hat{X}_{k}^{\pi_{k}^{*, \mathfrak{n}}}(T)\right) \mid \hat{X}_{k}^{\pi_{k}^{*, \mathfrak{n}}}(t)=x, L(t)=l\right] \\
= & \mathbb{E}\left[W^{k, \mathfrak{n}}\left(T, \hat{X}_{k}^{\pi_{k}^{*, \mathfrak{n}}}(T), L(T)\right) \mid \hat{X}_{k}^{\pi_{k}^{*, \mathfrak{n}}}(t)=x, L(t)=l\right] \\
= & \lim _{i \rightarrow \infty} \mathbb{E}\left[W^{k, \mathfrak{n}}\left(\tau_{i} \wedge T, \hat{X}_{k}^{\pi_{k}^{*, \mathfrak{n}}}\left(\tau_{i} \wedge T\right), L\left(\tau_{i} \wedge T\right)\right) \mid \hat{X}_{k}^{\pi_{k}^{*, \mathfrak{n}}}(t)=x, L(t)=l\right]=W^{k, \mathfrak{n}}(t, x, l),
\end{aligned}
$$

establishing (15).

\section{References}

[1] Asmussen, S., Højgaard, B., Taksar, M., (2000). "Optimal risk control and dividend distribution policies: example of excess-of-loss reinsurance for an insurance corporation". Finance and Stochastics 4, 299-324.

[2] Bai, L.H., Cai, J., Zhou, M., (2013). "Optimal reinsurance policies for an insurer with a bivariate reserve risk process in a dynamic setting". Insurance: Mathematics and Economics 53, 664-670.

[3] Bensoussan, A., Frehse, J. (2000). "Stochastic games for $N$ players". Journal of Optimization Theory and Applications 105(3), 543-565.

[4] Bensoussan, A., Friedman, A. (1977). "Nonzero-sum stochastic differential games with stopping times and free boundary problem." Transactions of the American Mathematical Society 231(2), 275-327.

[5] Bensoussan, A., Siu, C. C., Yam, S. C. P., Yang, H. (2014) "A class of nonzero-sum stochastic differential investment and reinsurance games." Automatica 50(8), 20252037. 
[6] Browne, S., (1995). "Optimal investment policies for a firm with a random risk process: exponential utility and minimizing the probability of ruin." Mathematics of Operations Research 20, 937-958.

[7] Browne, S. (2000). "Stochastic differential portfolio games." Journal of Applied Probability 37, 126-147.

[8] Bühlmann, H. (1970). Mathematical Methods in Risk Theory. Springer, Berlin.

[9] Cox, J. C., Ingersoll, J. E., Ross, S. A, (1985). "A theory of term structure of interest rates." Econometrica 53, 385-407.

[10] Elliott, R. J. (1976). "The existence of value in stochastic differential games." SIAM Journal of Control and Optimization 14(1), 85-94.

[11] Elliott, R. J., Siu, T. K. (2011). "A BSDE approach to a risk-based optimal investment of an insurer." Automatica 47 (2), 253-261.

[12] Espinosa, G.-E., Touzi, N. (2015). "Optimal investment under relative performance concerns." Mathematical Finance 25 (2), 221-257.

[13] Ethier, S. N., Kurtz, T. G. (1987). Markov Processes. Wiley, New York.

[14] Gerber, H.U., 1979. An introduction to Mathematical Risk Theory. University of Pennsylvania, Philadelphia.

[15] Hamadéne, S., Zhang, J. (2009). 'The Continuous Time Nonzero-sum Dynkin Game Problem and Application in Game Options." SIAM Journal of Control E Optimizations 48(5), 3659-3669.

[16] Hamadéne, S., Hassani, M. (2014). 'The Multi-player Nonzero-sum Dynkin Game in Continuous Time." SIAM Journal of Control \& Optimizations 52 (2) 821-835.

[17] Heston, S. L., (1993). "A closed-form solution for options with stochastic volatility with applications to bond and currency options." Review of Financial Studies 6, 327-343.

[18] Kraft, H. (2005). "Optimal portfolios and Heston's stochastic volatility model: an explicit solution for power utility." Quantitative Finance 5(3), 303-313.

[19] Liu, Y., Ma, J. (2009). "Optimal reinsurance/investment problems for general insurance models." The Annals of Applied Probability 19 (4), 1495-1528.

[20] Liu, J., Yiu, C. K-F., Siu, T. K., Ching, W. K. (2013). "Optimal investment-reinsurance with dynamic risk constraint and regime switching." Scandinavian Actuarial Journal 2013 (4), 263-285.

[21] Liu, J., Yiu, C. K-F., Siu, T. K. (2014). "Optimal investment of an insurer with regimeswitching and risk constraint." Scandinavian Actuarial Journal 2014 (7), 583-601.

[22] Ren, Y.-F. (2008). "On the Burkholder-Davis-Gundy inequalities for continuous martingales." Statistics and Probability Letters 78, 3034-3039. 
[23] Rolski, T., Schmidli, H., Schmidt, V., Teugels, J. (1999). Stochastic Processes for Insurance and Finance. John Wiley \& Sons.

[24] Sato, K. (1999). Lévy Processes and Infinitely Divisible Distributions. Cambridge University Press.

[25] Taskar, M., Zeng, X. D. (2013). "A general stochastic volatility model and optimal portfolio selection." Quantitative Finance 13(10), 1547-1558.

[26] Tijs., S. H. (1981). "Nash equilibria for noncooperative n-person games in normal form." SIAM Review 23, 225-237.

[27] Zeng, X., (2010). "A stochastic differential reinsurance game." Journal of Applied Probability 47(3), 335-349.

[28] Zhao, H., Rong, X., Zhao, Y. (2013). "Optimal excess-of-loss reinsurance and investment problemf or an insurer with jump-diffusion risk process under Heston model.". Insurance: Mathematics and Economics 53, 504-514. 